Supporting Information

for

\title{
Vinyl iodide containing polymers directly prepared via an iodo-yne polymerization
}

Joseph A. Jaye ${ }^{\dagger}$ and Ellen M. Sletten ${ }^{*,+}$

${ }^{+}$Department of Chemistry and Biochemistry, University of California, Los Angeles, 607 Charles E. Young, Dr. E., Los Angeles, CA 90095, USA.

*Prof. Ellen M. Sletten: sletten@chem.ucla.edu 


\section{Table of Contents}

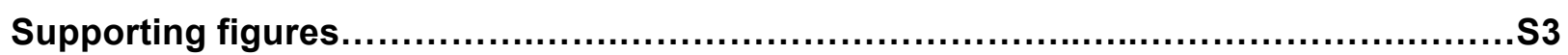

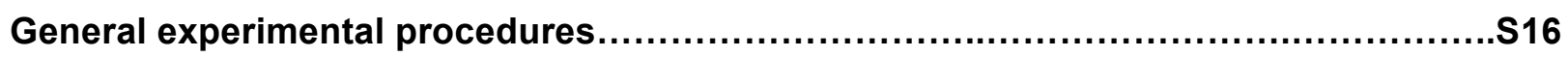

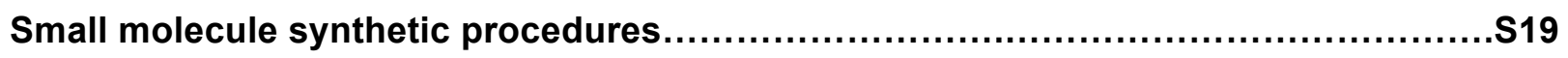

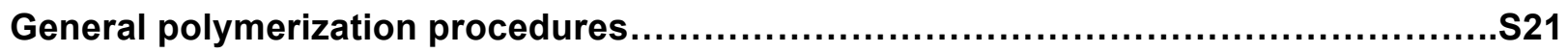

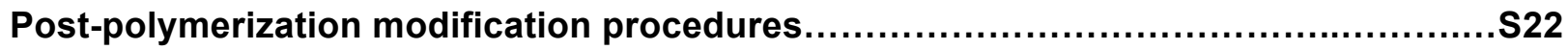

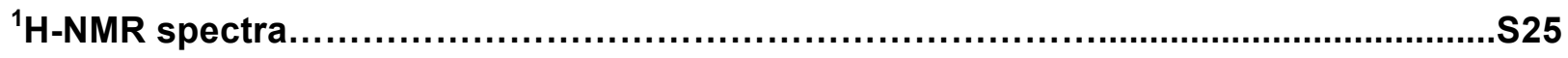

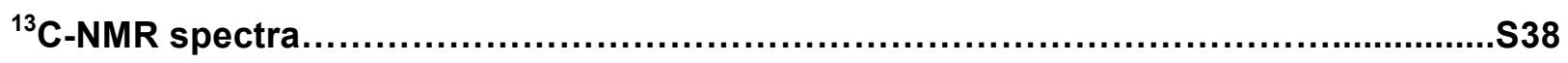

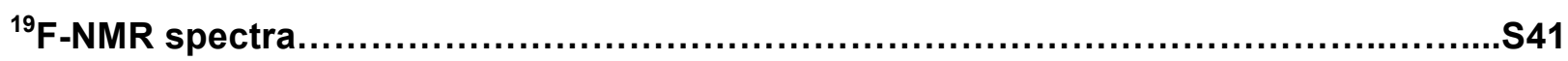

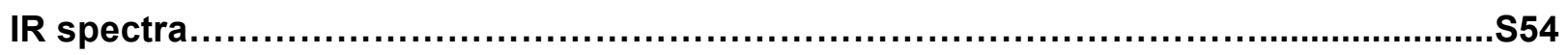

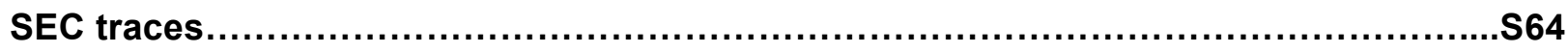

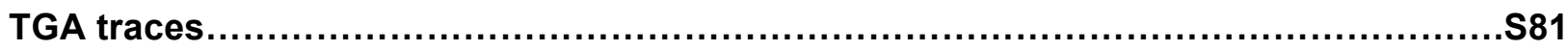

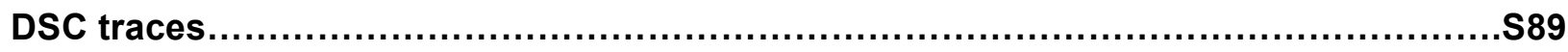

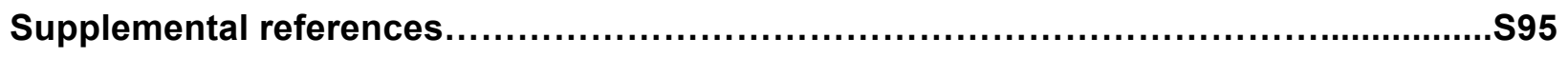




\section{A) Model reaction of 1-octyne and diiodoperfluorohexane}

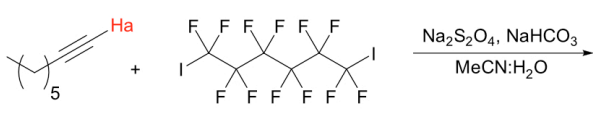

\section{B) HR GC-MS}
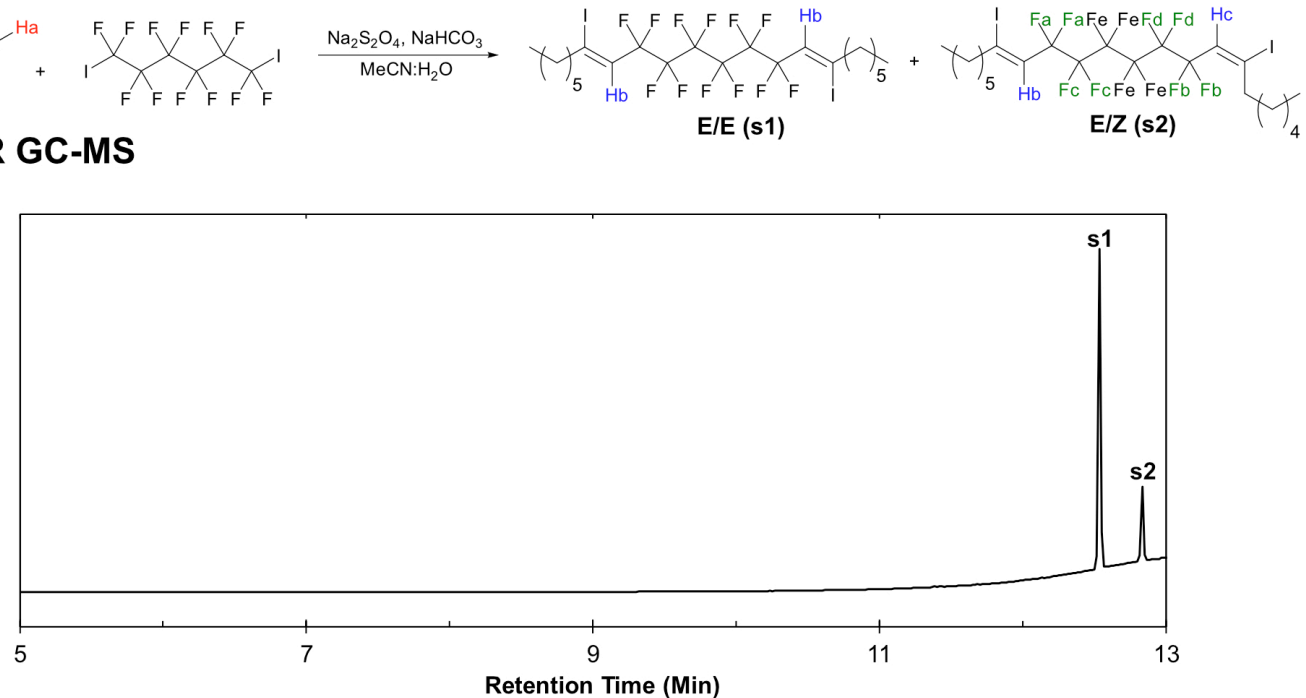

\section{C) ${ }^{1} \mathrm{H}-\mathrm{NMR}$}

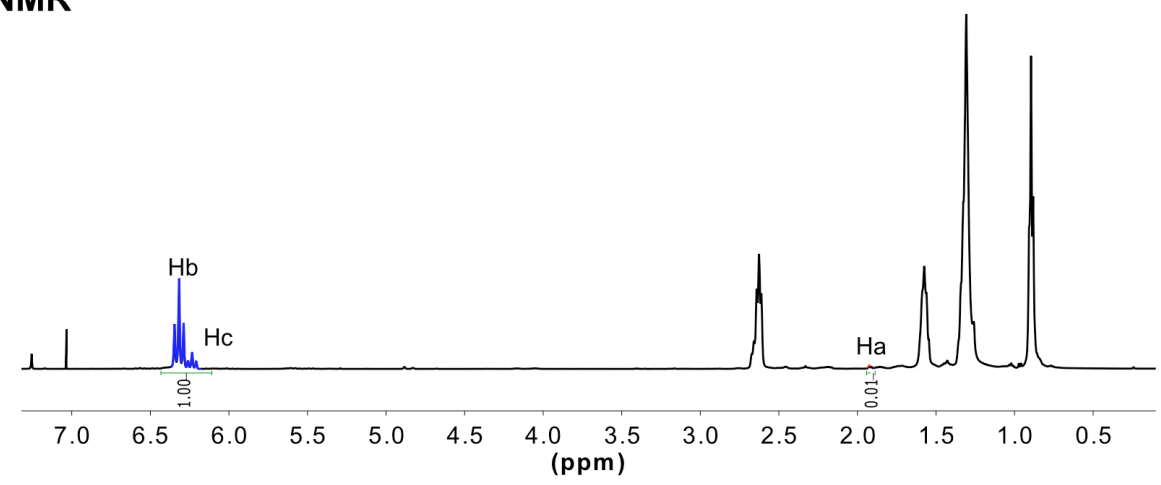

\section{D) ${ }^{19}$ F-NMR}

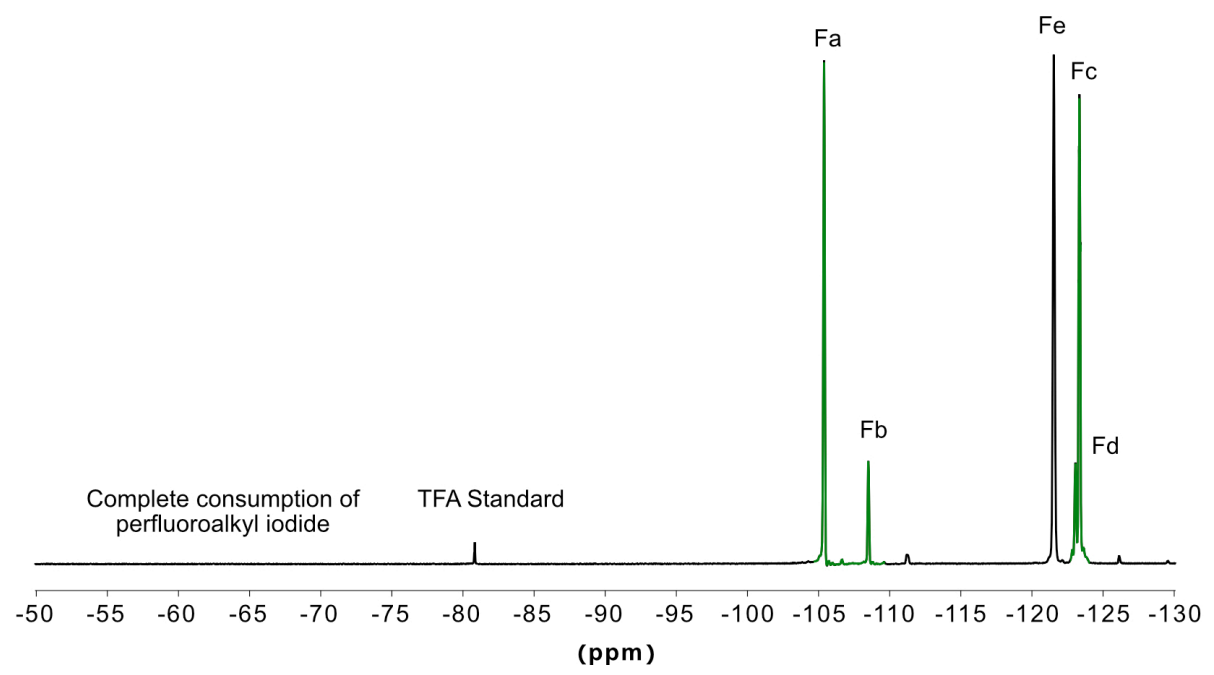

Figure S1: Diiodoperfluorohexane and 1-Octyne model system for polymerization. A) Reaction scheme of model system that gives a mix of diastereomers. B) High resolution GCMS of diastereomers. C) ${ }^{1} \mathrm{H}-$ NMR demonstrating nearly quantitative conversion of 1-octyne and diiodoperfluorohexane. D) ${ }^{19} \mathrm{~F}-\mathrm{NMR}$ demonstrating nearly quantitative conversion of 1-octyne and diiodoperfluorohexane. 


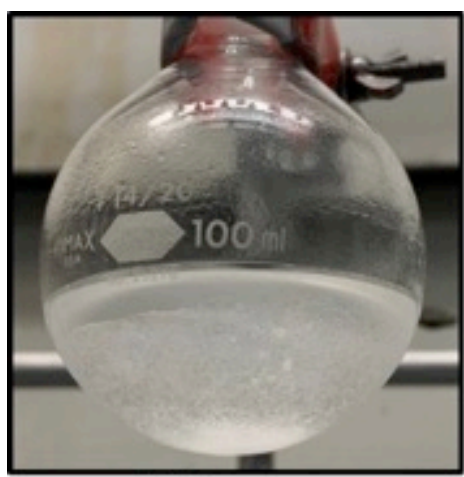

0 Minutes

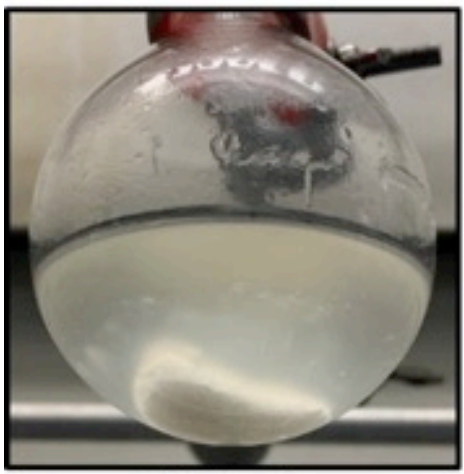

15 Minutes

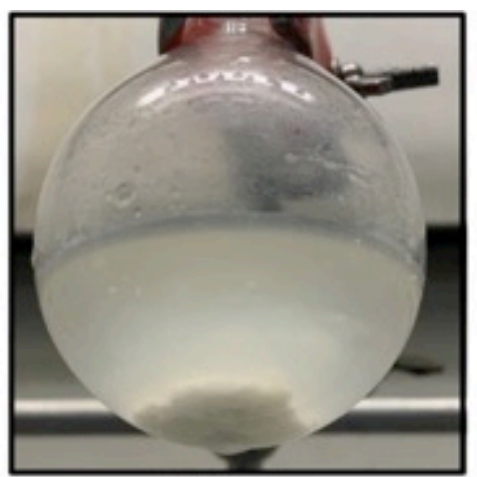

1 Minute

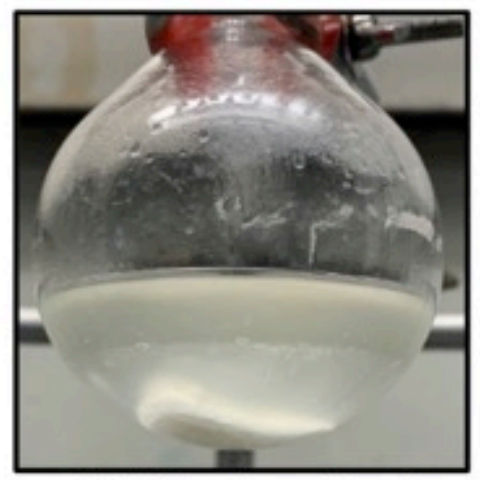

120 Minutes - Crude

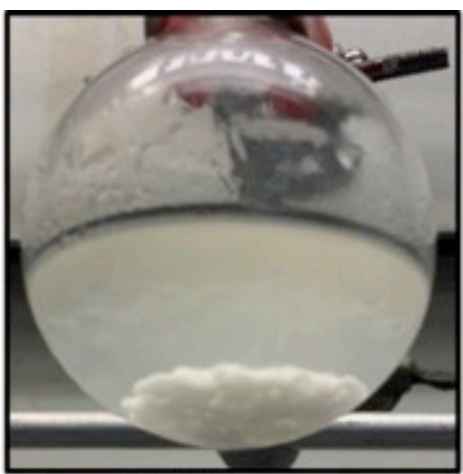

5 Minutes

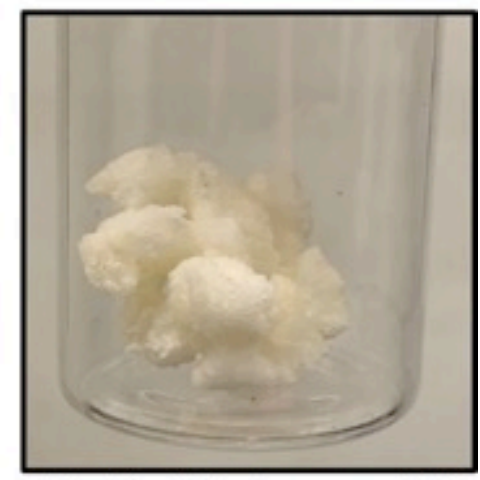

Purified Polymer - 2.3 grams, $94 \%$ Yield

Figure S2: Time lapse of large scale polymerization of diiodoperfluorohexane and 1,9-decadiyne. Purified polymer yielded 2.3 grams, a $94 \%$ yield. 
A)

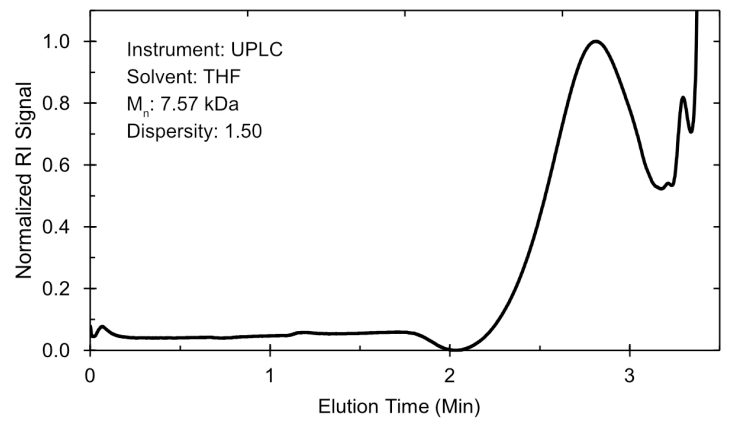

C)

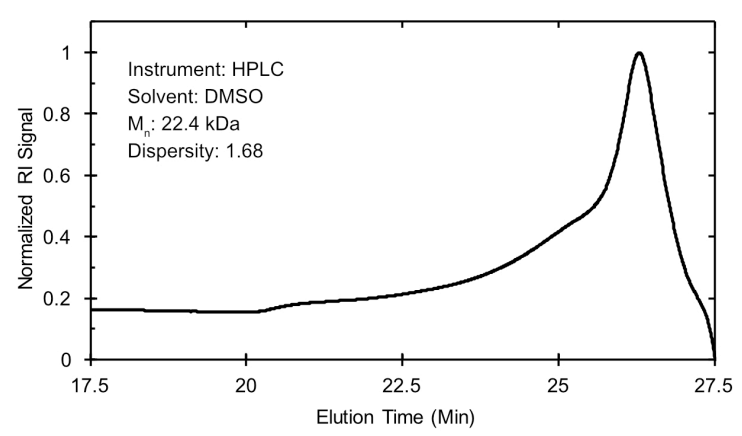

B)

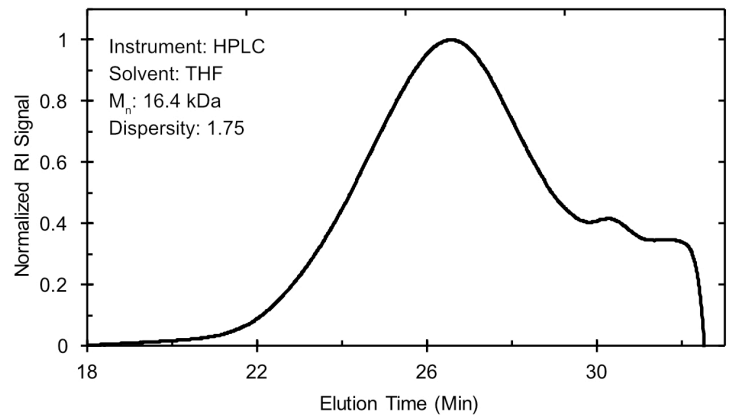

D)

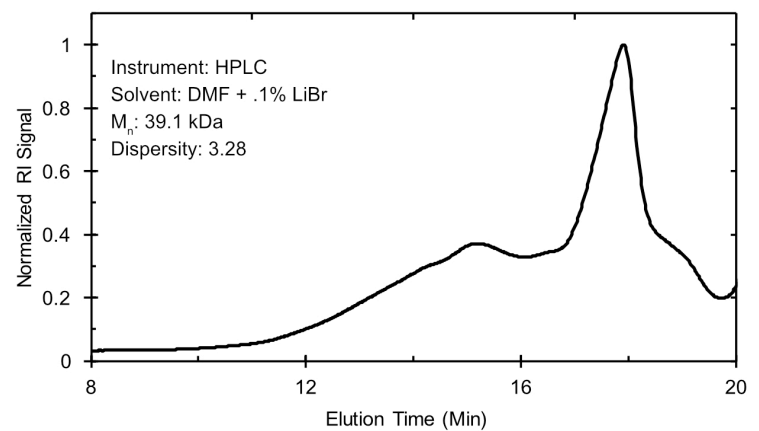

Figure S3: Comparison of iodo-yne polymer molecular weight calculation through different SEC anaylsis conditions. All molecular weight data was calculated through refractive index signal. A) SEC performed with THF as an eluent at $39{ }^{\circ} \mathrm{C}$ with a flowrate of $0.9 \mathrm{~mL} / \mathrm{min}$ at 6900 PSI. The instrument was calibrated with a set of low dispersity polystyrene standards. B) SEC performed with THF as eluent at $50{ }^{\circ} \mathrm{C}$ with a flowrate of $0.7 \mathrm{~mL} / \mathrm{min}$. The instrument was calibrated with a set of low dispersity polystyrene standards. C) SEC performed with DMSO as eluent at $65^{\circ} \mathrm{C}$ with a flowrate of $0.3 \mathrm{~mL} / \mathrm{min}$. The instrument was calibrated with a set of low dispersity poly(methyl methacrylate) standards. D) SEC performed with $\mathrm{DMF}+0.1 \% \mathrm{LiBr}$ as eluent at $50^{\circ} \mathrm{C}$ with a flowrate of $1.0 \mathrm{~mL} / \mathrm{min}$. The instrument was calibrated with a set of low dispersity polystyrene standards. 
A)

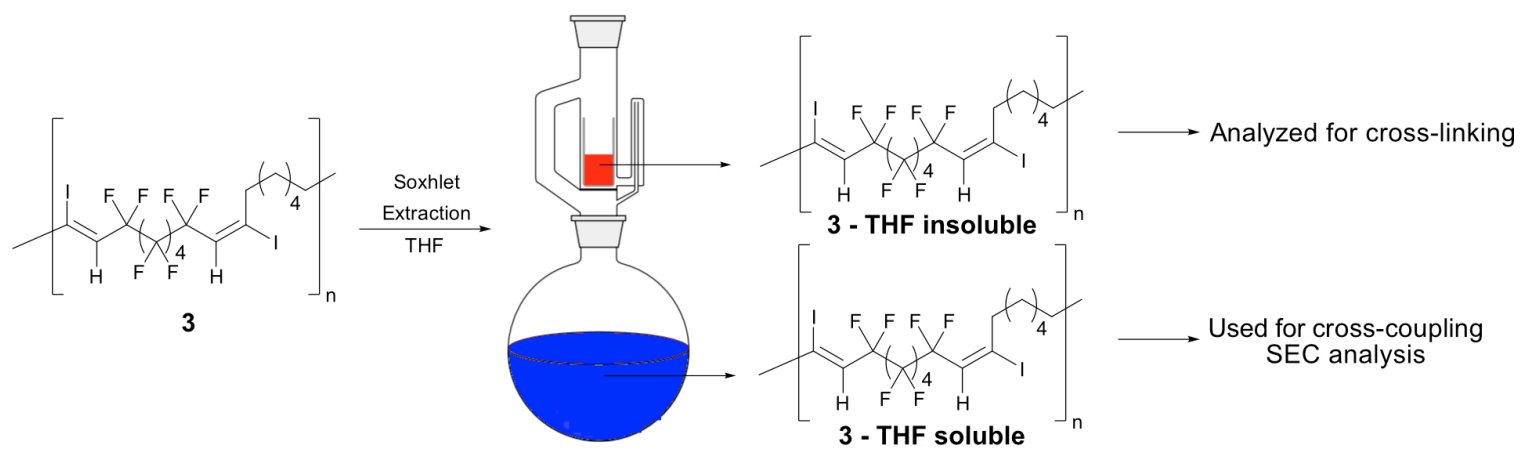

B)

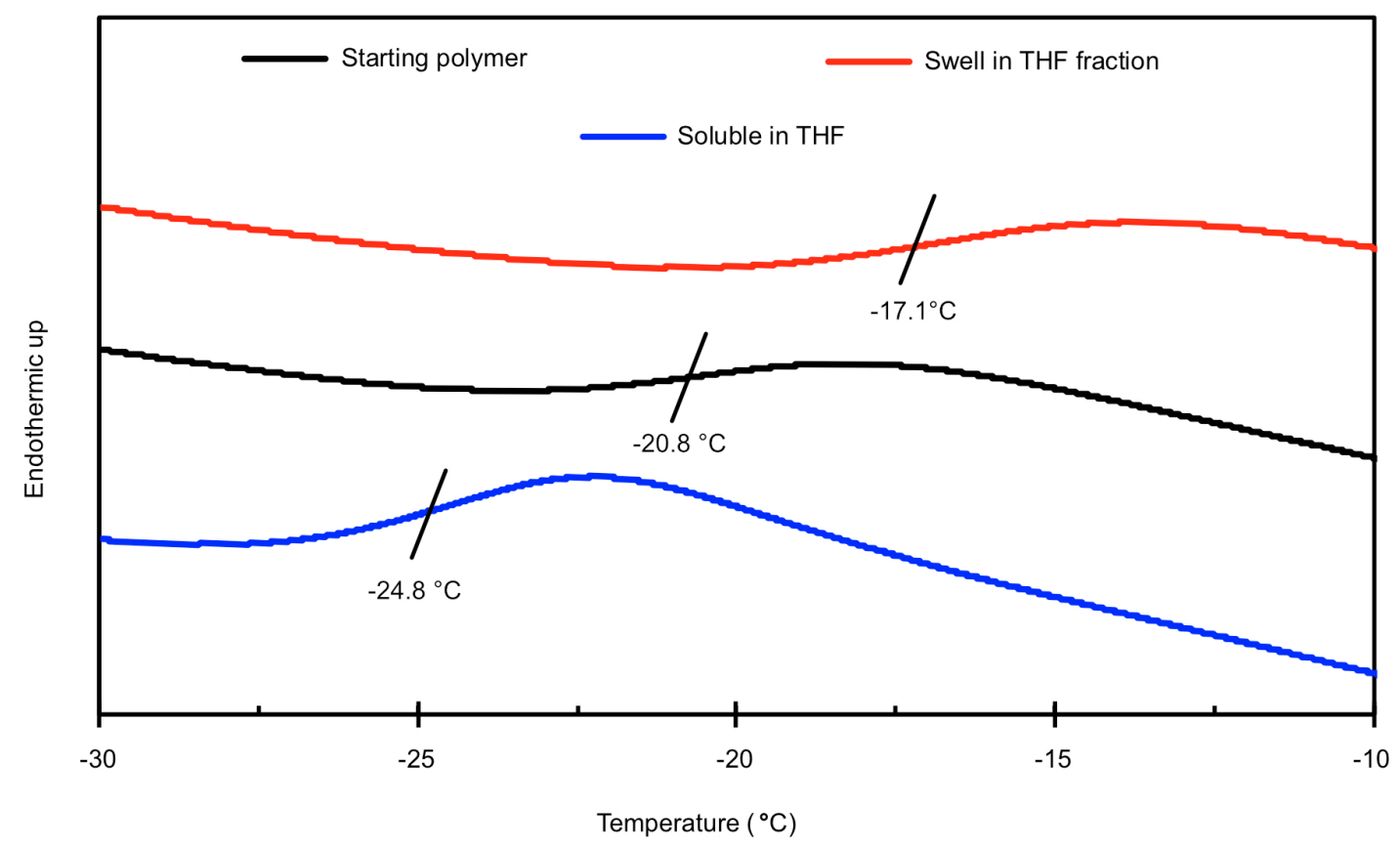

Figure S4: Soxhlet fractionation of polymer $\mathbf{3}$ and analysis via differential scanning calorimetry. A) Scheme representing Soxhlet extraction of THF soluble polymer 3. B) Differential scanning calorimetry comparison of iodo-yne polymer $\mathbf{3}$ before and after Soxhlet purification in THF. Polymer $\mathbf{3}$ before Soxhlet purification (middle, black), the THF soluble fraction of polymer $\mathbf{3}$ (bottom, blue) and the THF insoluble fraction of polymer $\mathbf{3}$ (top, red). 
A) ${ }^{1} \mathrm{H}-\mathrm{NMR}$

Polymer 3
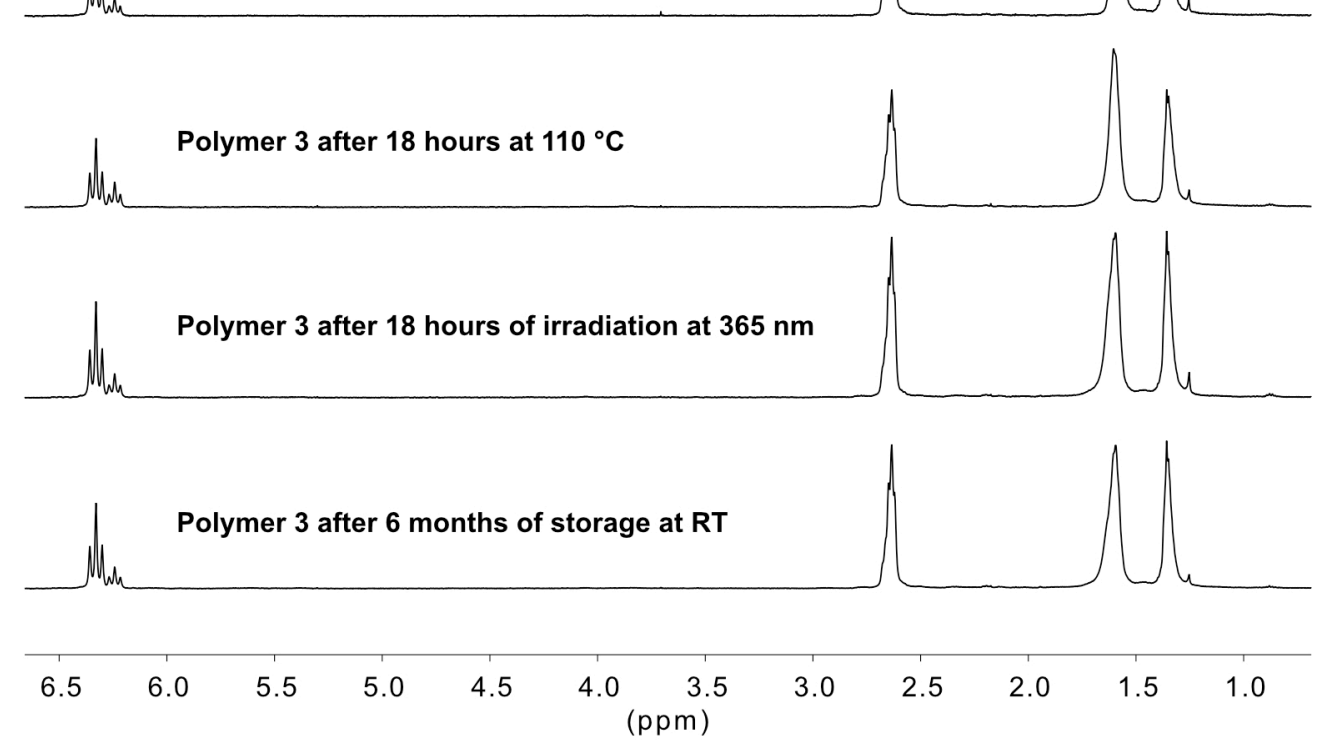

B) ${ }^{19} \mathrm{~F}-\mathrm{NMR}$

Polymer 3
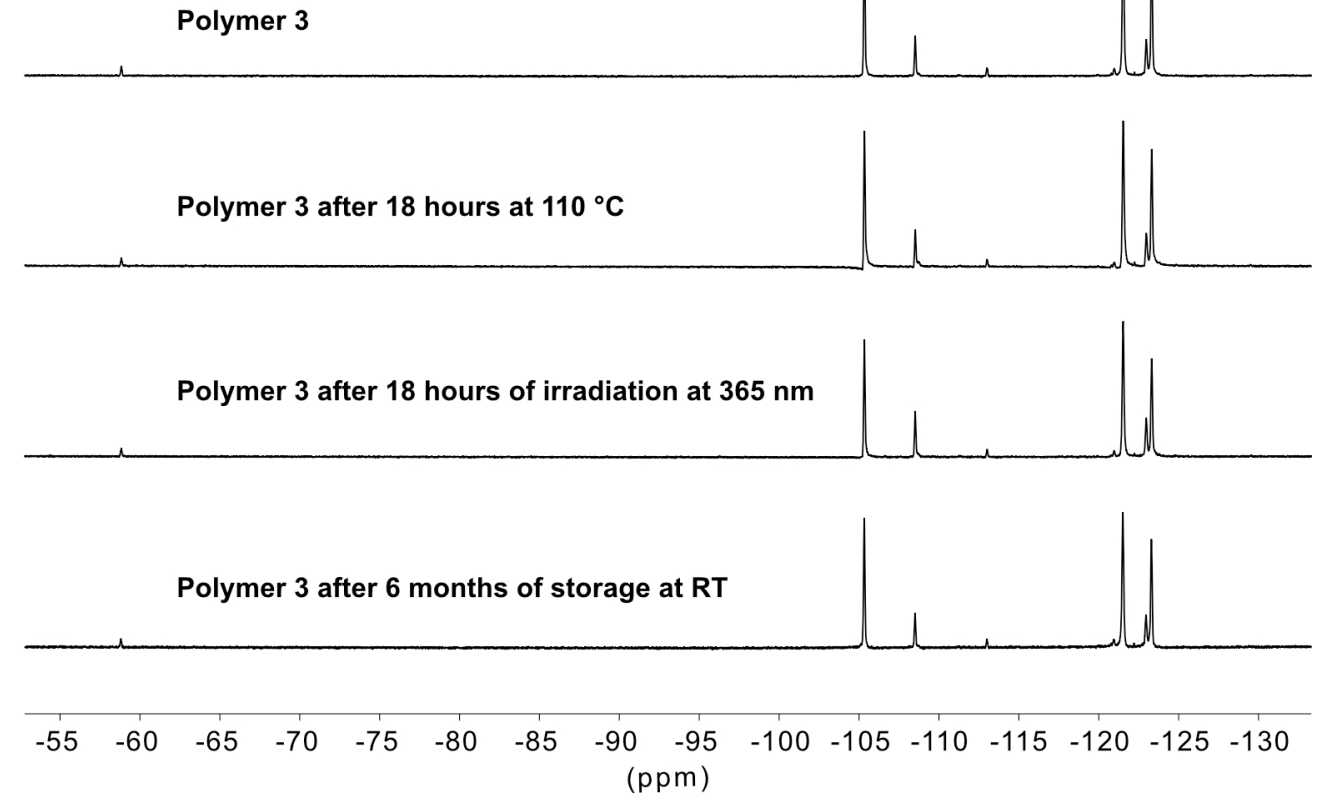

Figure S5: NMR analysis of polymer 3 following exposure to heat, light, or prolonged shelf life. A) ${ }^{1} \mathrm{H}$ NMR. B) ${ }^{19}$ F-NMR. 
A)

Polymer 3 before cross-link

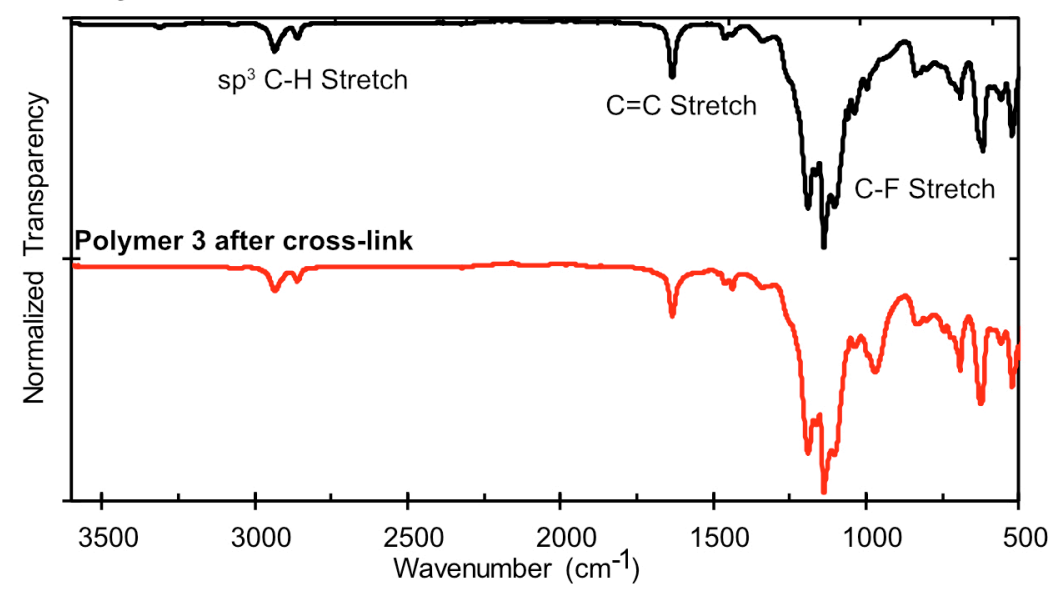

B)

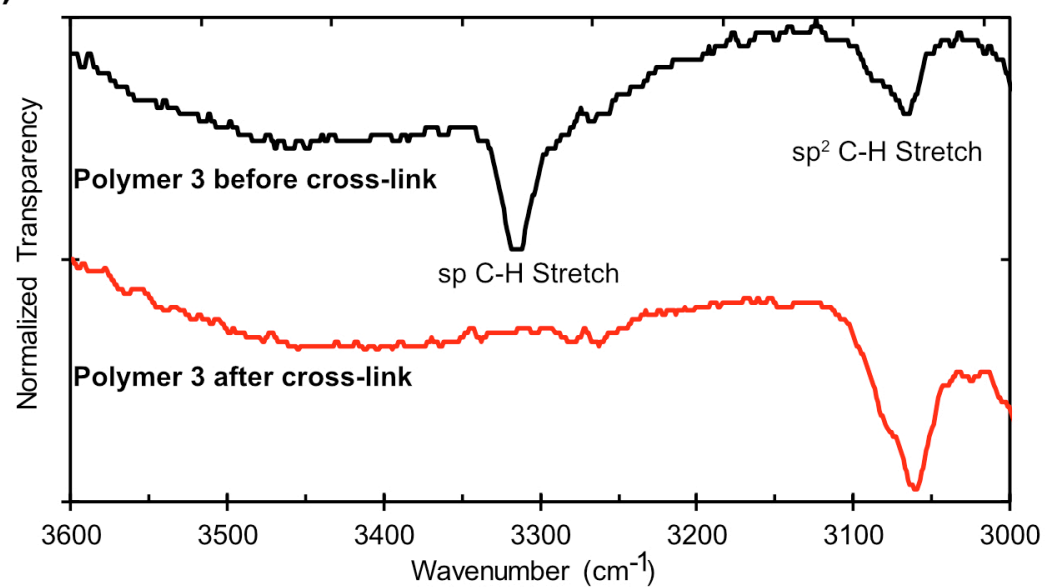

Figure S6: Characterization of polymer 3 treated with $\mathrm{Pd}\left(\mathrm{PPh}_{3}\right)_{4}$. A) Infrared spectroscopy (IR) spectra of polymer $\mathbf{3}$ before (black, top) and after (red, bottom) treatment. B) Enhanced region of the identical infrared spectroscopy (IR) spectra in A, revealing complete loss of terminal alkyne C-H stretch due to reaction between vinyl iodide groups and terminal alkyne groups. 


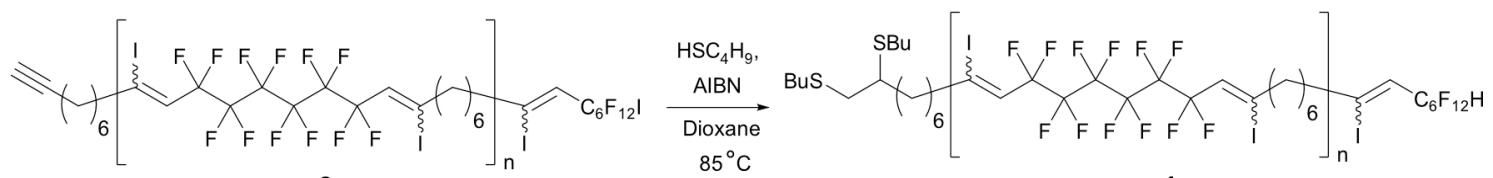

4

Figure S7: Reaction scheme of AIBN initiated thiol-yne addition of butanethiol to polymer $\mathbf{3}$ to give polymer 4. Both possible end groups are shown at the polymer termini to demonstrate the reactions of each functionality. The polymer mixture likely also includes polymers with two terminal perfluoroiodides or two terminal alkynes. 


\section{A) Workflow of polymer purification}

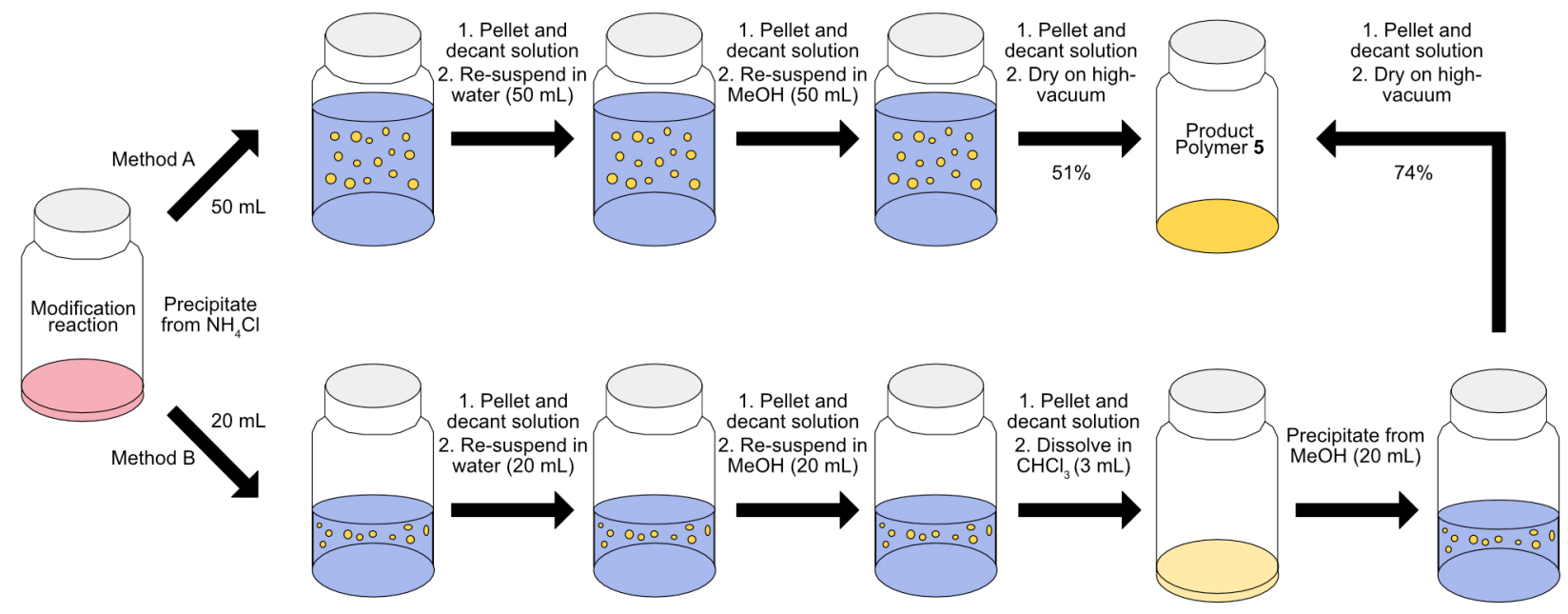

B) Stacked ${ }^{1} \mathrm{H}-\mathrm{NMR}$ of pure Polymer 5 and the concentrated work-up solution

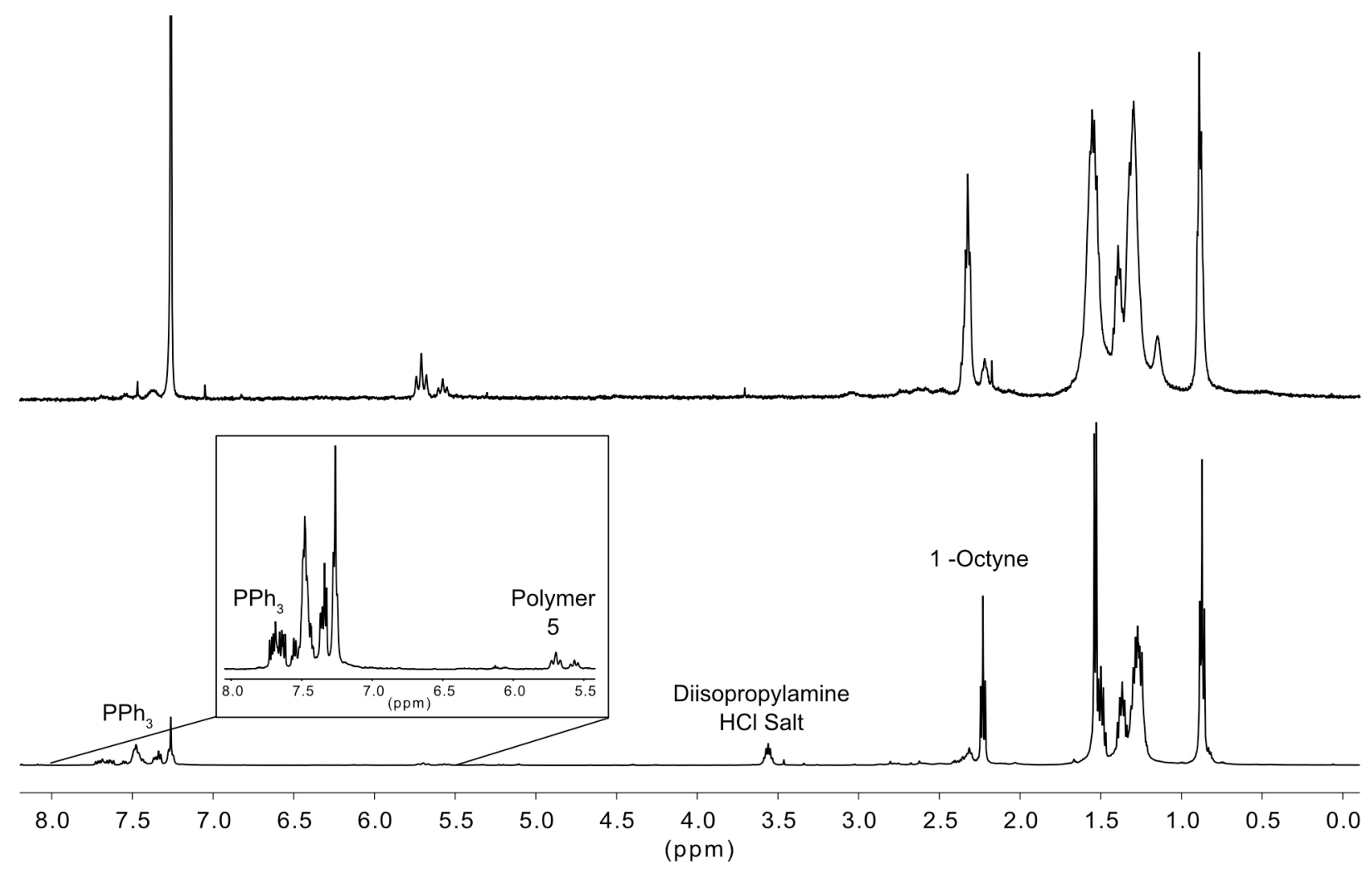

Figure S8: Optimization of purification conditions of the Sonogashira coupling to give polymer 5. A) Unoptimized purification conditions to give polymer 5 in $51 \%$ yield (top, method A) and the optimized conditions which gave polymer 5 in $74 \%$ yield (bottom, method B). B) Stacked ${ }^{1} \mathrm{H}-\mathrm{NMR}$ of the purified polymer 5 (top) and the concentrated methanol work-up containing 1-octyne, triphenylphosphine, diisopropylammonium chloride, and extracted polymer $\mathbf{5}$ (bottom). 


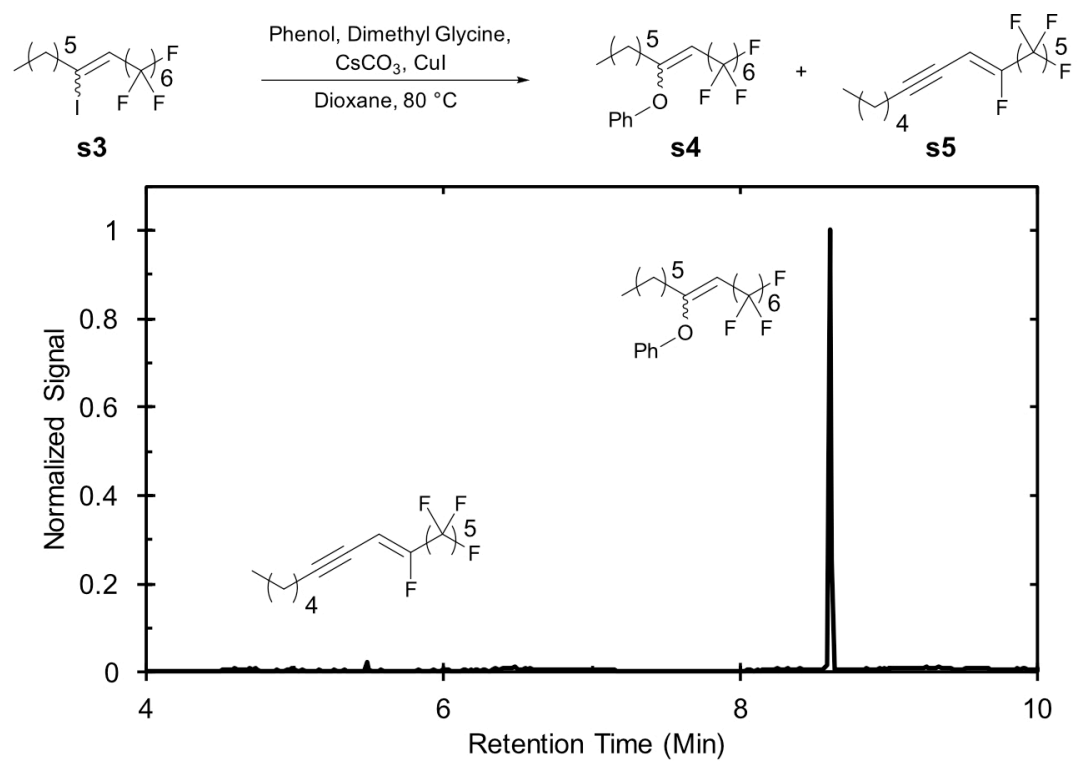

Figure 59: Small molecule analog to investigate the phenol coupling. Small molecule $\mathbf{s} 3$ when reacted with phenol and copper iodide employing dimethylglycine as the ligand to give the phenol coupled product $\mathbf{s} \mathbf{4}$ as the major product, with a small amount of ene-yne $\mathbf{s} 5$ formed as a by-product. 


\section{Polymer 5:}

\section{Polymer 6:}

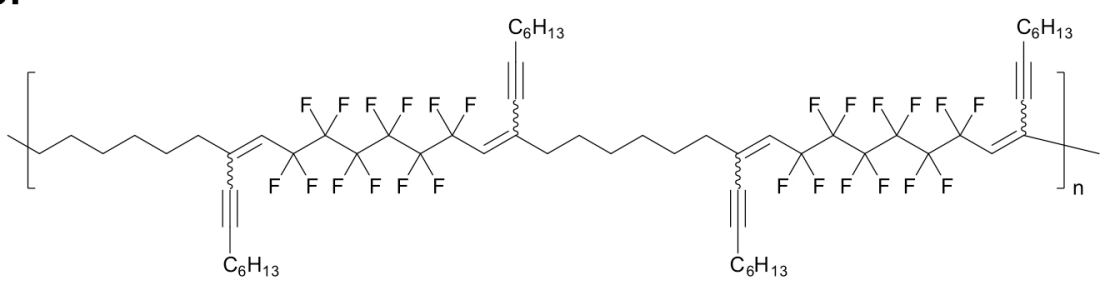

\section{Polymer 7:}
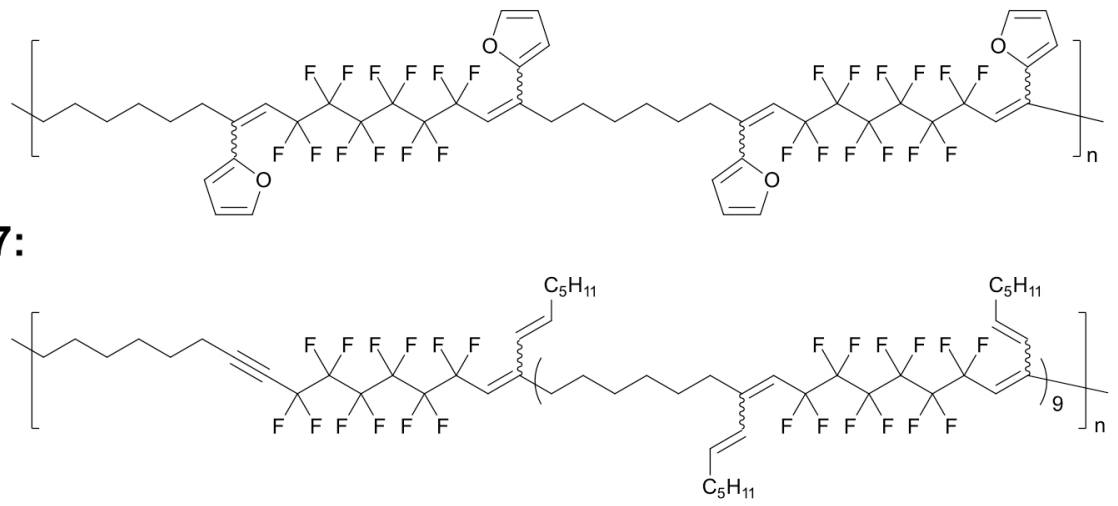

\section{Polymer 8:}

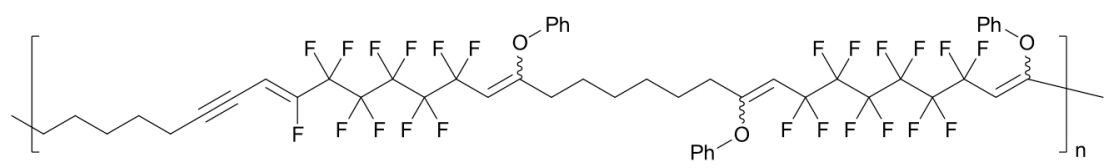

\section{Polymer 9:}

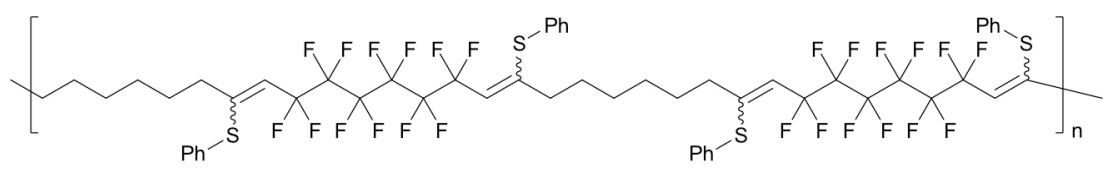

\section{Polymer 10:}

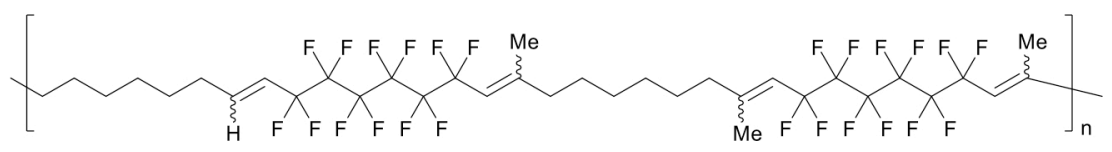

Figure S10: Representation of polymers synthesized in Table 2. 
A.

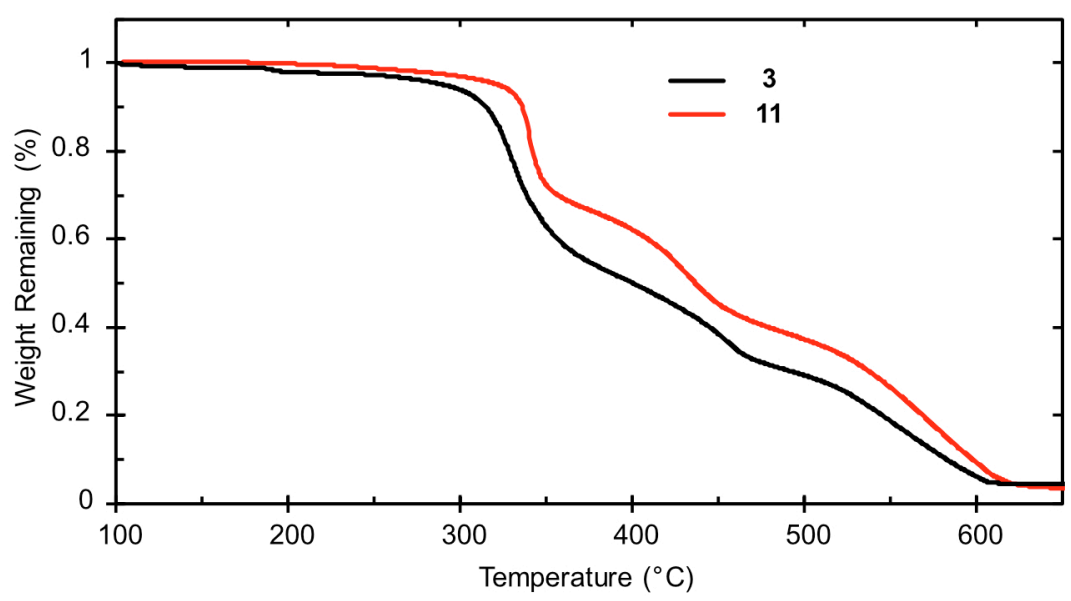

B.

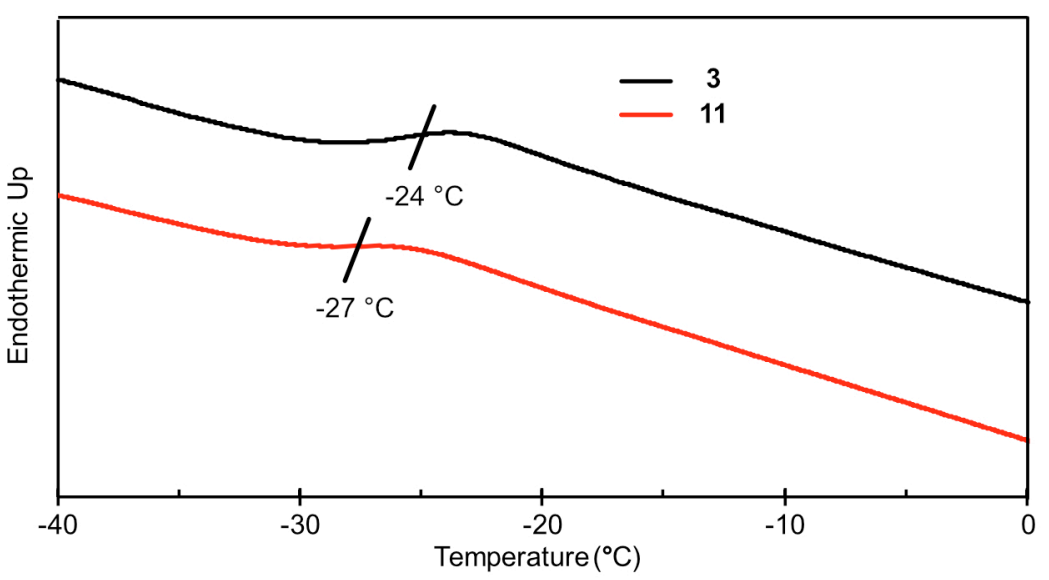

Figure S11: Thermal analysis of polymers 3 and 11. A) Thermal gravimetric analysis (TGA) of polymers 3 (black) and $\mathbf{1 1}$ (red) under oxygen, showing an increase in thermal stability following elimination of the iodide with tetramethyl guanidine. B) Differential scanning calorimetry (DSC) of polymers $\mathbf{3}$ (black) and 11 (red) indicating a minor change in the glass transition temperature $\left(T_{g}\right)$. 


\section{A) ${ }^{1} \mathrm{H}-\mathrm{NMR}$}

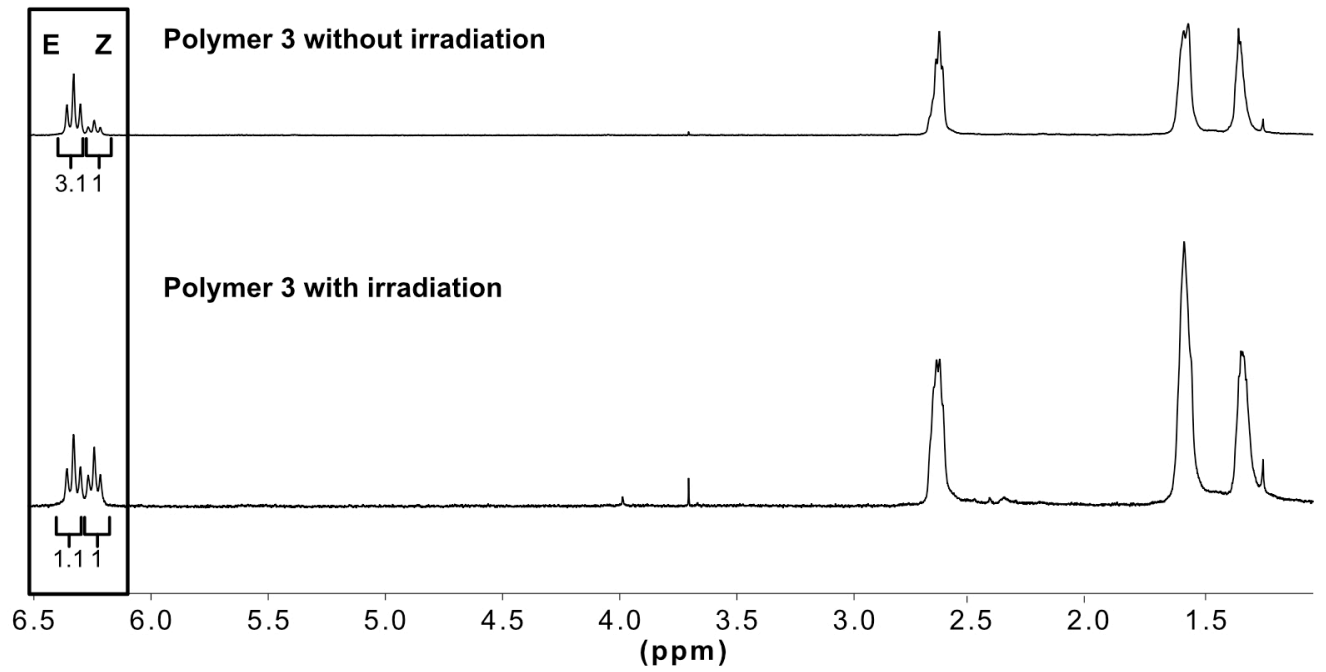

\section{B) ${ }^{19} \mathrm{~F}-\mathrm{NMR}$}

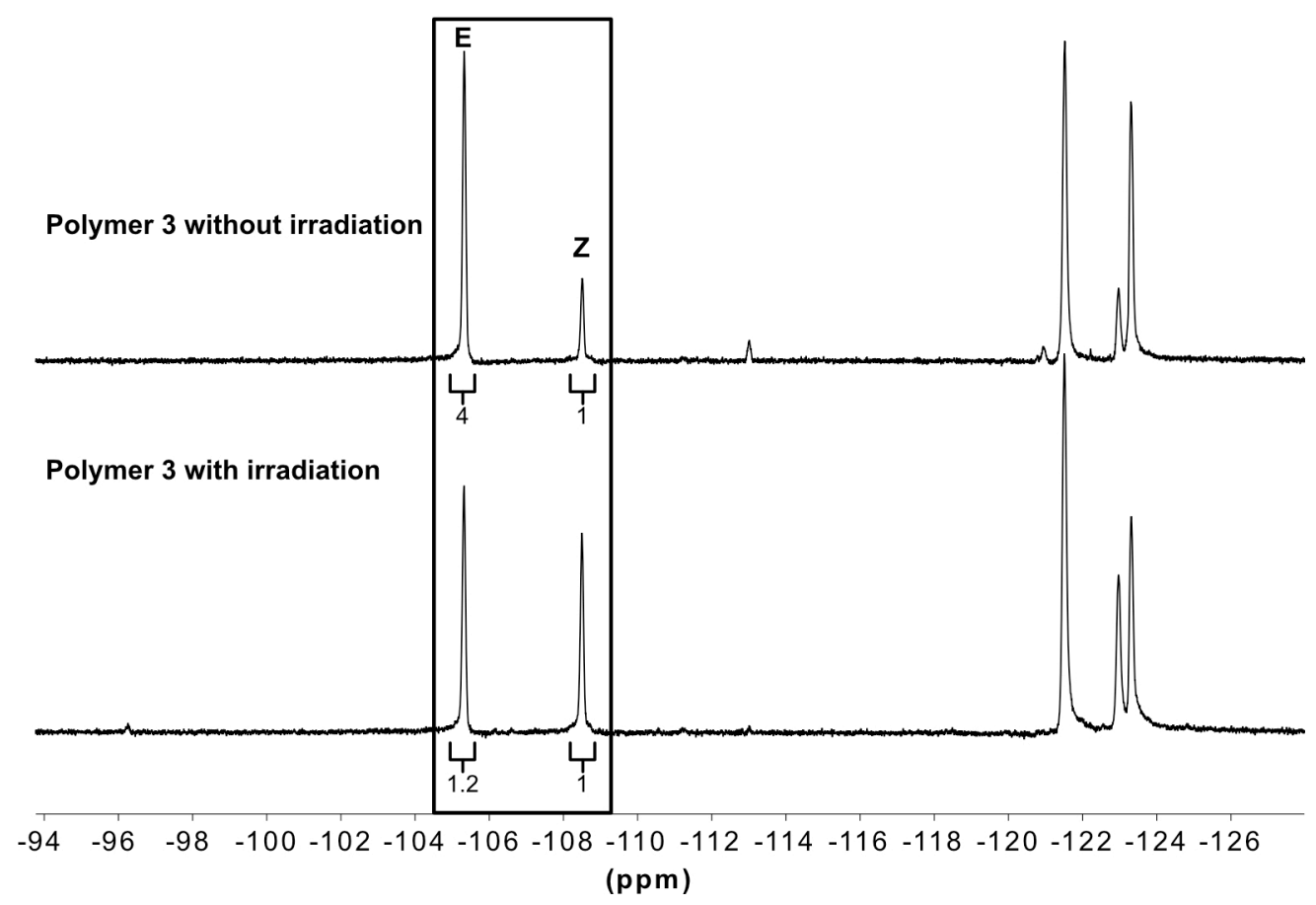

Figure S12: Use of $365 \mathrm{~nm}$ light to isomerize the olefin generated via iodo-yne polymerization. Polymer sample was irradiated while swollen in toluene solution at a concentration of $100 \mathrm{mg} / \mathrm{mL}$. A) ${ }^{1} \mathrm{H}-\mathrm{NMR}$ comparing polymer $\mathbf{3}$ that had not been irradiated with light (top) and polymer $\mathbf{3}$ that had been irradiated with light (bottom). B) ${ }^{19} \mathrm{~F}-\mathrm{NMR}$ comparing polymer $\mathbf{3}$ that had not been irradiated with light (top) and polymer $\mathbf{3}$ that had been irradiated with light (bottom). 

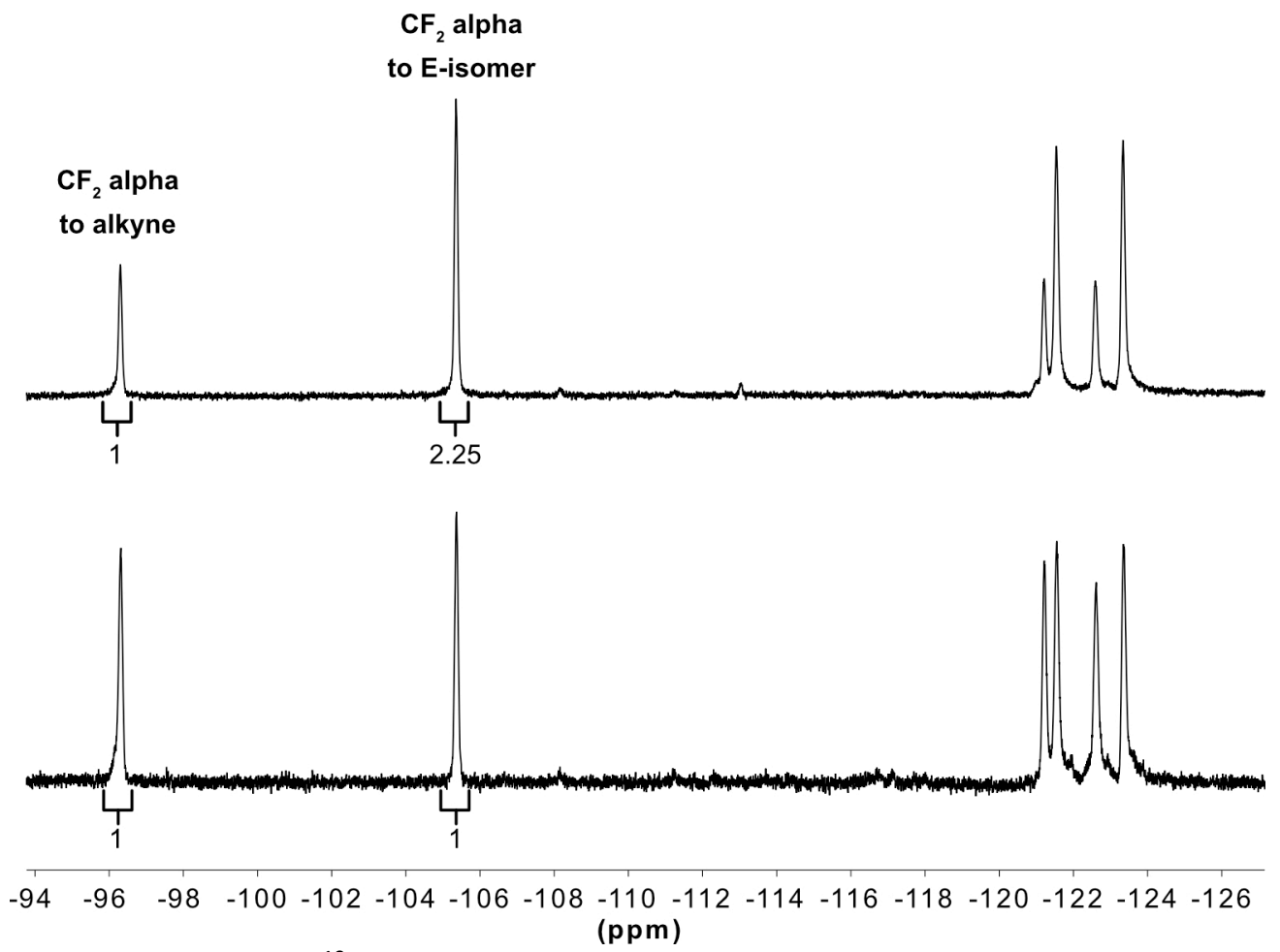

Figure S13: Overlaid ${ }^{19} \mathrm{~F}-\mathrm{NMR}$ spectra comparing the elimination of vinyl iodide with and without irradiation of $365 \mathrm{~nm}$ light. ${ }^{19} \mathrm{~F}-\mathrm{NMR}$ of polymer $\mathbf{1 1}$ that was synthesized with tetramethyl guanidine as a base, without $365 \mathrm{~nm}$ light (top) and ${ }^{19} \mathrm{~F}-\mathrm{NMR}$ of polymer $\mathbf{1 1}$ that was synthesized with tetramethyl guanidine as a base, with illumination via $365 \mathrm{~nm}$ light throughout the reaction (bottom). 


\section{General experimental procedures:}

Chemical reagents were purchased from Sigma-Aldrich, Alfa Aesar, Fisher Scientific, or Acros Organics and used without purification unless noted otherwise. No unexpected or unusually high safety hazards were encountered. Anhydrous and deoxygenated solvents toluene (PhMe), tetrahydrofuran (THF), dichloromethane (DCM), acetonitrile (MeCN), and dimethylformamide (DMF) were dispensed from a Grubb's-type Phoenix Solvent Drying System ${ }^{1}$. Thin layer chromatography was performed using Silica Gel 60 F254 (EMD Millipore) plates. Flash chromatography was executed with technical grade silica gel with $60 \AA$ pores and $40-63 \mu \mathrm{m}$ mesh particle size (Sorbtech Technologies). Solvent was removed under reduced pressure with a Büchi Rotovapor with a Welch self-cleaning dry vacuum pump and further dried with a Welch DuoSeal pump. Bath sonication was performed using a Branson 3800 ultrasonic cleaner. Nuclear magnetic resonance $\left({ }^{1} \mathrm{H}-\mathrm{NMR},{ }^{13} \mathrm{C}-\mathrm{NMR}\right.$, and $\left.{ }^{19} \mathrm{~F}-\mathrm{NMR}\right)$ spectra were taken on Bruker Avance $500\left({ }^{1} \mathrm{H}-\mathrm{NMR}\right.$ and $\left.{ }^{13} \mathrm{C}-\mathrm{NMR}\right)$ or $\mathrm{AV}-300\left({ }^{19} \mathrm{~F}-\mathrm{NMR}\right)$ instruments and processed with MestReNova software. All ${ }^{1} \mathrm{H},{ }^{13} \mathrm{C}$, and ${ }^{19} \mathrm{~F}$ NMR spectra are reported in ppm and relative to residual solvent signals $\left({ }^{1} \mathrm{H},{ }^{13} \mathrm{C}\right)$. Fluorine NMR were reported with trifluoroacetic acid as the reference peak as an external standard. Size exclusion chromatography (SEC), unless otherwise noted, was conducted on a Shimadzu prominence-I LC-2030C high performance liquid chromatography (HPLC) system with a UV detector and connected to a Wyatt Dawn Heleos-II light scattering detector and Wyatt Optilab T-rEX refractive index detector, one MZ Analysentechnik GPC-Precolumn $50 \times 8.0 \mathrm{~mm} \mathrm{MZ-Gel} \mathrm{SDplus} \mathrm{Linear} \mathrm{LS} 5 \mu \mathrm{m}$ pore, and two MZ Analysentechnik GPC-column $300 \times 8.0 \mathrm{~mm} \mathrm{MZ-Gel} \mathrm{SDplus} \mathrm{Linear} \mathrm{LS} 5 \mu \mathrm{m}$ pore. Eluent was THF at $50{ }^{\circ} \mathrm{C}$ (flow rate: $0.70 \mathrm{~mL} / \mathrm{min}$ ). Calibration was performed using near monodisperse polystyrene PS standards from Polymer Laboratories. Differential scanning calorimetry measurements were taken on a PerkinElmer DSC. Thermal gravimetric analysis was performed on a PerkinEImer Pyris Diamond TG/DTA Thermogravimetric/Differential Thermal Analyzer. Mass spectra (Electron impact (EI)) were collected on an Agilent 7890B7520 Quadrupole Time-of-Flight GC/MS. Irradiation with light was performed with BI365 nm Inspection UV LED lamp, purchased from Risk reactor (Output power density $>5000 \mu \mathrm{W} / \mathrm{cm}^{2}$ at $15 "$ (38 cm), voltage range $90-265 \mathrm{~V}$ ac, output power: $3^{*} 325 \mathrm{~mW}$ at $365 \mathrm{~nm}$ peak). Centrifugation was performed on a Thermo Scientific Sorvall ST 16 Centrifuge. All sonication was done in a Branson M-Series Model $3800120 \mathrm{~V}$ bath sonicator. For probe sonication, a QSonica (Q125) sonicator was used.

Abbreviations: AIBN = azoisobutyronitrile; $\mathrm{DBU}=1,8$-Diazabicyclo[5.4.0]undec-7-ene; $\mathrm{DCM}=$ dichloromethane; DMF = dimethylformamide; DMSO = dimethylsulfoxide; $\mathrm{DSC}=$ differential scanning calorimetry; $\mathrm{Et}_{2} \mathrm{O}=$ diethyl ether; $\mathrm{MeCN}=$ acetonitrile $; \mathrm{MeOH}=$ methanol; $\mathrm{PhMe}=$ toluene; $\mathrm{SEC}=$ size exclusion chromatography; $\mathrm{TGA}=$ thermal gravimetric analysis; $\mathrm{THF}=$ tetrahydrofuran; $\mathrm{TMG}=$ tetramethylguanine

\section{SEC prep/procedures:}

THF HPLC instrument: Size exclusion chromatography (SEC) was conducted on a Shimadzu prominence-I LC-2030C high performance liquid chromatography (HPLC) system with a UV detector and connected to a Wyatt Dawn Heleos-II light scattering detector and Wyatt Optilab T- 
rEX refractive index detector. Calibration was performed using near monodisperse polystyrene standards from Polymer Laboratories. Polymer samples were dissolved in THF $(5 \mathrm{mg} / \mathrm{mL})$ and stirred at $50{ }^{\circ} \mathrm{C}$ for 1 hour. Polymer solutions were filtered through 0.2 micron PTFE filter and 50 $\mu \mathrm{L}$ were then run through one $\mathrm{MZ}$ Analysentechnik GPC-Precolumn $50 \times 8.0 \mathrm{~mm} \mathrm{MZ-Gel}$ SDplus Linear LS $5 \mu \mathrm{m}$ pore, and two MZ Analysentechnik GPC-column $300 \times 8.0 \mathrm{~mm} \mathrm{MZ-Gel}$ SDplus Linear LS $5 \mu \mathrm{m}$ pore columns at $50{ }^{\circ} \mathrm{C}$ with an eluent rate of $0.7 \mathrm{~mL} / \mathrm{min}$. Unless otherwise noted, UV absorbance was used for molecular weight determination.

DMSO HPLC instrument: Size exclusion chromatography (SEC) was conducted on a Shimadzu prominence-I LC-2030C high performance liquid chromatography (HPLC) system with a UV detector and connected to a Wyatt Dawn Heleos-II light scattering detector and Wyatt Optilab T-rEX refractive index detector. Calibration was performed using near monodisperse poly (methyl-methacrylate) PMMA standards from Polymer Laboratories. Polymer samples were dissolved in DMSO $(5 \mathrm{mg} / \mathrm{mL})$ and stirred at $100{ }^{\circ} \mathrm{C}$ for 1 hour. Polymer solutions were filtered through 0.2 micron PTFE filter and $50 \mu \mathrm{L}$ were then run through one Agilent PLgel guard column D, and an Agilent PLgel $10 \mu \mathrm{m}$ mixed B columns at $65{ }^{\circ} \mathrm{C}$ with a flow rate at 0.35 $\mathrm{mL} / \mathrm{min}$. Unless otherwise noted, RI signal was used for molecular weight determination.

DMF HPLC instrument: Size exclusion chromatography (SEC) was conducted on a Waters Alliance HPLC System, 2695 Separation Module high performance liquid chromatography (HPLC) system with a Waters 2414 Differential Refractometer (RI) and Waters 2998 Photodiode Array Detector (PDA). Calibration was performed using near monodisperse polystyrene standards from Polymer Laboratories. Polymer samples were dissolved in DMF $(5 \mathrm{mg} / \mathrm{mL})$ and stirred at $85^{\circ} \mathrm{C}$ for 1 hour. Polymer solutions were filtered through 0.2 micron PTFE filter and 40 $\mu \mathrm{L}$ were then run through 2 Tosoh TSKgel Super HM-M columns at $50{ }^{\circ} \mathrm{C}$ with a flow rate at $0.30 \mathrm{~mL} / \mathrm{min}$. Unless otherwise noted, RI signal was used for molecular weight determination.

THF UPLC instrument: Size exclusion chromatography (SEC) was conducted on a Waters Acquity APC ultra-high performance liquid chromatography (UPLC) system with an ACQUITY UPLC PDA Detector and an ACQUITY UPLC Refractive Index Detector. Calibration was performed using near monodisperse polystyrene standards from Polymer Laboratories. Polymer samples were dissolved in THF $(5 \mathrm{mg} / \mathrm{mL})$ and stirred at $50{ }^{\circ} \mathrm{C}$ for 1 hour. Polymer solutions were filtered through 0.2 micron PTFE filter and $25 \mu \mathrm{L}$ were then run through Three Acquity APC XT Columns $(45+200+450$ pore sizes $)$ at $39{ }^{\circ} \mathrm{C}$ with a flow rate at $0.9 \mathrm{~mL} / \mathrm{min}$ with a column pressure of $6900 \mathrm{PSI}$. Unless otherwise noted, RI signal was used for molecular weight determination.

TGA prep/procedures: Polymer samples $(5-10 \mathrm{mg})$ were placed in a calibrated ceramic container and the temperature was raised to $100{ }^{\circ} \mathrm{C}$. After a delay of 1 minute to remove residual solvent, the weight of the sample was re-recorded, and the temperature was raised to $650{ }^{\circ} \mathrm{C}$ at a rate of $20{ }^{\circ} \mathrm{C} / \mathrm{min}$. The resulting data were then normalized to \% weight loss of sample. Unless otherwise noted all samples were run under nitrogen atmosphere.

DSC prep/procedures: Polymer sample $(10-20 \mathrm{mg})$ were placed in an aluminum pan and cooled to $-50{ }^{\circ} \mathrm{C}$ and equilibrated for 2 minutes. The samples were then heated to $100{ }^{\circ} \mathrm{C}$ at a 
rate of $20^{\circ} \mathrm{C} / \mathrm{min}$ with a 2-minute pause at $100{ }^{\circ} \mathrm{C}$. Samples were then cooled back down to -50 ${ }^{\circ} \mathrm{C}$ at a rate of $15{ }^{\circ} \mathrm{C} / \mathrm{min}$ with a 2-minute pause at $-50{ }^{\circ} \mathrm{C}$. This cycle was then repeated two additional times.

Photochemistry assembly: Our homemade photobox was assembled to the shape of the UV light source (Risk reactor (Output power density $>5000 \mu \mathrm{W} / \mathrm{cm}^{2}$ at 15 " $(38 \mathrm{~cm}$ ), voltage range 90 $265 \mathrm{~V}$ ac, output power: $3^{*} 325 \mathrm{~mW}$ at $365 \mathrm{~nm}$ peak)) using cardboard and black tape. The interior was then coated with aluminum foil and holes were cut on the top sample placement.

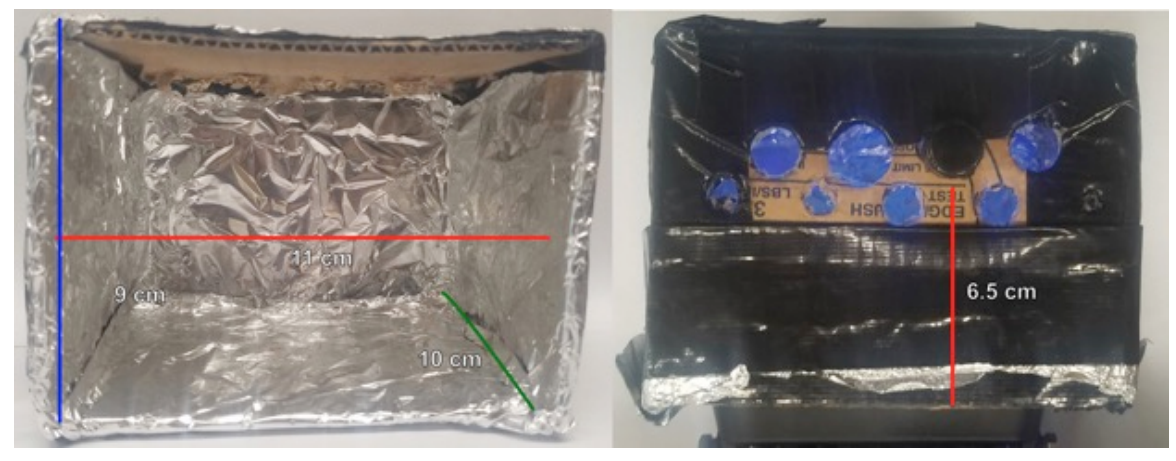




\section{Small molecule procedures:}

\section{9,9,10,10,11,11,12,12,13,13,14,14-dodecafluoro-7,16-diiododocosa-7,15-diene, s1 (E,E)}

and $s 2(E, Z)$ :

Diiodoperfluorohexane $(1.1 \mathrm{~g}, 2.0 \mathrm{mmol}, 1.0 \mathrm{eq})$ was dissolved in acetonitrile $(8 \mathrm{~mL})$ and water $(6 \mathrm{~mL})$. 1-Octyne $(0.451 \mathrm{~g}, 4.09 \mathrm{mmol}, 2.1 \mathrm{eq})$ was added, followed by sodium bicarbonate $(0.39 \mathrm{~g}, 4.6 \mathrm{mmol}, 2.3 \mathrm{eq})$. Sodium dithionite $(0.80 \mathrm{~g}, 4.6 \mathrm{mmol}, 2.3 \mathrm{eq})$ was then added and the solution was placed in a sonication bath for 2 hours. The reaction mixture was diluted with brine $(10 \mathrm{~mL})$ and extracted with EtOAc $(3 \times 15 \mathrm{~mL})$. The organic layer was dried with $\mathrm{MgSO}_{4}$, decanted, and concentrated to give crude oil. Purification through silica plug with hexanes as the eluent yielded the product as a clear oil and mix of isomers $(0.83 \mathrm{~g}, 1.3 \mathrm{mmol}, 65 \%) .{ }^{1} \mathrm{H}$ NMR $\left(\mathrm{CDCl}_{3}, 500 \mathrm{MHz}\right), \delta \mathrm{ppm}: 6.31(\mathrm{t}, J=12 \mathrm{~Hz}, 1.6 \mathrm{H}), 6.23(\mathrm{t}, J=12 \mathrm{~Hz}, 0.4 \mathrm{H}), 2.62(\mathrm{~m}$, $4 \mathrm{H}), 1.57(\mathrm{~m}, 4 \mathrm{H}), 1.30(\mathrm{~m}, 12 \mathrm{H}), 0.9(\mathrm{t}, J=8 \mathrm{~Hz}, 6 \mathrm{H}) .{ }^{13} \mathrm{C} \mathrm{NMR}\left(\mathrm{CDCl}_{3}, 126 \mathrm{MHz}\right), \delta \mathrm{ppm}$ : $126.7(\mathrm{t}, J=25.2 \mathrm{~Hz}), 122.8(\mathrm{t}, J=6.3 \mathrm{~Hz}), 121.8$ (t, $J=25.2 \mathrm{~Hz}), 117.0-107.0,(\mathrm{~m}, 7 \mathrm{C}), 41.1$, 31.5, 30.0, 29.0, 28.1, 27.6, 22.5, 14.0. $\left.{ }^{19} \mathrm{~F} \mathrm{NMR} \mathrm{(} \mathrm{CDCl}_{3}, 376 \mathrm{MHz}\right), \delta \mathrm{ppm}:-105.35$ (s, 4F), 108.46 (s, 0.8F), -121.51 (s, 4.8F), -123.00 (s, 0.8F), -123.29 (s, 4F). HRMS (EI) Calculated for $\mathrm{C}_{22} \mathrm{H}_{28} \mathrm{~F}_{12} \mathrm{I}_{2}\left[\mathrm{M}-\mathrm{I}^{-}\right]:$: 774.0089, found: 774.0069 .

\section{1,1,1,2,2,3,3,4,4,5,5,6,6-tridecafluoro-8-iodotetradec-7-ene, s3:}

lodoperfluorohexane $(1.5 \mathrm{~g}, 3.3 \mathrm{mmol}, 1.1 \mathrm{eq})$ was dissolved in acetonitrile $(6 \mathrm{~mL})$ and water $(4.5 \mathrm{~mL})$. 1-Octyne $(0.33 \mathrm{~g}, 3.0 \mathrm{mmol}, 1.0 \mathrm{eq})$ was added followed by sodium bicarbonate $(0.32$ $\mathrm{g}, 3.8 \mathrm{mmol}, 1.2 \mathrm{eq})$. Sodium dithionite $(0.66 \mathrm{~g}, 3.8 \mathrm{mmol}, 1.2 \mathrm{eq})$ was then added and the solution was placed in a sonication bath for 2 hours. The reaction mixture diluted with brine (10 $\mathrm{mL})$ and extracted with EtOAc $(3 \times 15 \mathrm{~mL})$. The organic layer was dried with $\mathrm{MgSO}_{4}$, decanted, and concentrated to give crude oil. Purification through a silica plug with hexanes as the eluent gave pure product as a clear oil $(1.38 \mathrm{~g}, 2.71 \mathrm{mmol}, 82 \%)$. NMR data matched known compound. $^{2}$

\section{$((9,9,10,10,11,11,12,12,13,13,14,14,14$-tridecafluorotetradec-7-en-7-yl)oxy)benzene, s4:}

Compound s3 $(0.52 \mathrm{~g}, 0.93 \mathrm{mmol}, 1.0 \mathrm{eq})$ was dissolved in dioxane $(6.5 \mathrm{~mL})$. Phenol $(0.350 \mathrm{~g}$, $3.92 \mathrm{mmol}, 4.0 \mathrm{eq})$ and dimethyl glycine $(0.028 \mathrm{~g}, 0.28 \mathrm{mmol}, 0.30 \mathrm{eq})$ were then added. The solution was degassed via freeze-pump-thaw $(x 3)$. Cesium carbonate $(0.636 \mathrm{~g}, 1.81 \mathrm{mmol}, 2.0$ eq) and copper iodide $(0.017 \mathrm{~g}, 0.093 \mathrm{mmol}, 0.10 \mathrm{eq})$ were then added and the reaction was set to $90{ }^{\circ} \mathrm{C}$ for 16 hours. The reaction was cooled to room temperature, concentrated and run through a silica column with hexanes as the eluent to give a mixture of compounds $\mathbf{s} 4$ and $\mathbf{s} 5$ in a $4: 1$ ratio as a clear oil $(0.210 \mathrm{~g}, 0.402 \mathrm{mmol}, 43 \%)$. This mixture was further separated by preparatory TLC. ${ }^{1} \mathrm{H}$ NMR $\left(\mathrm{CDCl}_{3}, 500 \mathrm{MHz}\right), \delta \mathrm{ppm}: 7.40(\mathrm{t}, \mathrm{J}=8.0 \mathrm{~Hz}, 2 \mathrm{H}), 7.23(\mathrm{t}, \mathrm{J}=7.4 \mathrm{~Hz}$, $1 \mathrm{H}), 7.01(\mathrm{~d}, J=7.5 \mathrm{~Hz}, 2 \mathrm{H}), 4.41(\mathrm{t}, J=14.9 \mathrm{~Hz}, 1 \mathrm{H}), 2.52(\mathrm{t}, J=7.7 \mathrm{~Hz}, 2 \mathrm{H}), 1.72(\mathrm{p}, J=7.6$

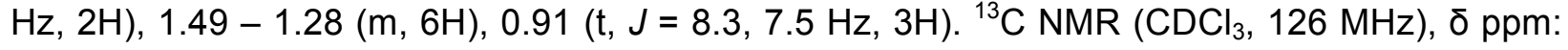
$170.1,153.5,130.1,125.6,121.4,119.4-107.2(\mathrm{~m}, 6 \mathrm{C}), 92.1(\mathrm{t}, J=25.2 \mathrm{~Hz}), 31.5,31.4(\mathrm{t}, J=$ $2.5 \mathrm{~Hz}), 28.9,27.4,22.5,14.0 .^{19} \mathrm{~F} \mathrm{NMR}\left(\mathrm{CDCl}_{3}, 376 \mathrm{MHz}\right), \delta \mathrm{ppm}: \delta-80.81(\mathrm{t}, J=9.9 \mathrm{~Hz}, 3 \mathrm{~F})$, $102.56(\mathrm{q}, J=13.6 \mathrm{~Hz}, 2 \mathrm{~F}),-121.67(\mathrm{~m}, 2 \mathrm{~F}),-122.86(\mathrm{~m}, 2 \mathrm{~F}),-123.38(\mathrm{~m}, 2 \mathrm{~F}),-126.14(\mathrm{~m}, 2 \mathrm{~F})$. HRMS (El) Calculated for $\mathrm{C}_{20} \mathrm{H}_{19} \mathrm{~F}_{13} \mathrm{O}\left[\mathrm{M}-\mathrm{HOC}_{6} \mathrm{H}_{5}\right]: 428.0810$, found: 428.0633 . 


\section{1,1,1,2,2,3,3,4,4,5,5,6-dodecafluorotetradec-6-en-8-yne, s5:}

Compound s3 $(0.42 \mathrm{~g}, 0.75 \mathrm{mmol}, 1.0 \mathrm{eq})$ was dissolved in dioxane $(4.2 \mathrm{~mL})$. Phenol $(0.28 \mathrm{~g}$, $3.0 \mathrm{mmol}, 4.0 \mathrm{eq})$ and 3,4,7,8-tetramethyl phenanthroline $(0.053 \mathrm{~g}, 0.23 \mathrm{mmol}, 0.30 \mathrm{eq})$ were then added. The solution was sparged under nitrogen gas for 20 minutes. Cesium carbonate $(0.53 \mathrm{~g}, 1.5 \mathrm{mmol}, 2.0 \mathrm{eq})$ and copper iodide $(0.014 \mathrm{~g}, 0.075 \mathrm{mmol}, 0.10 \mathrm{eq})$ were then added and the reaction was set to $90{ }^{\circ} \mathrm{C}$ for 16 hours. The reaction was cooled to room temperature, concentrated and run through a long silica column with hexanes as the eluent to give a mixture of compounds with predominately the en-yne rearrangement product $\mathbf{s} 5$ as a 3:1 ratio of $E / Z$ isomers, but with a small fraction of starting material and phenol coupled product which couldn't be removed $(0.080 \mathrm{~g}, 0.20 \mathrm{mmol}, 26 \%)$. ${ }^{1} \mathrm{H}$ NMR $\left(\mathrm{CDCl}_{3}, 500 \mathrm{MHz}\right), \delta \mathrm{ppm}$ : $\delta 6.09-5.99(\mathrm{~m}$, $0.25 \mathrm{H}), 5.71(\mathrm{dt}, J=29.3,2.1 \mathrm{~Hz}, 0.75 \mathrm{H}), 2.39(\mathrm{t}, J=6.7 \mathrm{~Hz}, 1.5 \mathrm{H}), 2.33(\mathrm{t}, J=6.9 \mathrm{~Hz}, 0.5 \mathrm{H})$, $1.61-1.54(\mathrm{~m}, 2 \mathrm{H}), 1.43-1.22(\mathrm{~m}, 4 \mathrm{H}), 0.91(\mathrm{t}, J=7.2 \mathrm{~Hz}, 3 \mathrm{H}) \cdot{ }^{13} \mathrm{C} \mathrm{NMR}\left(\mathrm{CDCl}_{3}, 126 \mathrm{MHz}\right), \delta$ ppm: $152.2(\mathrm{dt}, J=272.3,28.3 \mathrm{~Hz}), 119.6-107.3(\mathrm{~m}, 6 \mathrm{C}), 103.4(\mathrm{~d}, J=6.7 \mathrm{~Hz}), 98.2(\mathrm{q}, J=$ $6.4 \mathrm{~Hz}), 69.3,31.0,27.9,22.1,19.7,13.9 .{ }^{19} \mathrm{~F} \mathrm{NMR}\left(\mathrm{CDCl}_{3}, 376 \mathrm{MHz}\right), \delta \mathrm{ppm}:-80.83$ (s, 3F), $115.91--116.87(\mathrm{~m}, 0.5 \mathrm{~F}),-118.02(\mathrm{q}, J=13.8 \mathrm{~Hz}, 1.5 \mathrm{~F}),-118.42--119.16(\mathrm{~m}, 0.75 \mathrm{~F})$, $120.12--120.91(\mathrm{~m}, 0.25 \mathrm{~F}),-122.47--123.73(\mathrm{~m}, 4 \mathrm{~F}),-126.23(2 \mathrm{~F})$. Calculated for $\mathrm{C}_{14} \mathrm{H}_{12} \mathrm{~F}_{12}$ [M]: 408.0747, found: 408.0577 . 


\section{Polymerization procedures:}

\section{1-perfluorohexyl-2,9-diiodo-1,9-decadiene block polymer, 3:}

Diiodoperfluorohexane $(2.0 \mathrm{~g}, 3.6 \mathrm{mmol}, 1.0 \mathrm{eq})$ was dissolved in acetonitrile $(14.4 \mathrm{~mL})$ and water $(11.0 \mathrm{~mL})$. 1,9-Decadiyne $(0.48 \mathrm{~g}, 3.6 \mathrm{mmol}, 1.0 \mathrm{eq})$ was added, followed by sodium bicarbonate $(0.70 \mathrm{~g}, 8.3 \mathrm{mmol}, 2.3 \mathrm{eq})$. Sodium dithionite $(1.44 \mathrm{~g}, 8.27 \mathrm{mmol}, 2.3 \mathrm{eq})$ was added and the solution was placed in a sonication bath for 2 hours. The reaction mixture was then precipitated from water $(100 \mathrm{~mL})$ and washed with methanol $(2 \times 100 \mathrm{~mL})$. The precipitate was centrifuged at $2500 \times g$ for 5 minutes, and the resulting pellet was dried under high vacuum at $60{ }^{\circ} \mathrm{C}$ to yield a white solid $(2.33 \mathrm{~g}, 3.38 \mathrm{mmol}, 94 \%) .{ }^{1} \mathrm{H} \mathrm{NMR}\left(\mathrm{CDCl}_{3}, 500 \mathrm{MHz}\right), \delta \mathrm{ppm}$ : $6.33(\mathrm{t}, J=14.4 \mathrm{~Hz}, 1.5 \mathrm{H}), 6.24(\mathrm{t}, J=13.0 \mathrm{~Hz}, 0.5 \mathrm{H}), 2.65(\mathrm{~m}, 4 \mathrm{H}), 1.59(\mathrm{~m}, 4 \mathrm{H}), 1.45-1.27$ (m, 4H). ${ }^{19} \mathrm{~F} \mathrm{NMR}\left(\mathrm{CDCl}_{3}, 376 \mathrm{MHz}\right), \delta \mathrm{ppm}$ : -105.35 (s, 3F), -108.53 (s, 1F), -121.52 (s, 4F), 122.91 (s, 1F), -123.27(s, 3F). FT-IR: 2932 (C-H str) (w), 1635 (C=C) Vinyl iodide (s), 11001200 (C-F bend) (vs). TGA: $10 \%$ mass loss at $330{ }^{\circ} \mathrm{C}$. $T_{g}$ (DSC): $-24.0{ }^{\circ} \mathrm{C}$

\section{Fractionation between THF soluble and insoluble polymer 3 :}

Polymer $3(0.176 \mathrm{~g}, 0.254 \mathrm{mmol}$, repeat unit eq) was placed in a Soxhlet extraction apparatus lined with a cellulose thimble connected to a round bottom flask charged with THF without inhibitor $(100 \mathrm{~mL})$. THF was then brought to reflux for 16 hours. The THF layer was concentrated and dried under high vacuum to give THF soluble polymer 3 (0.105 g, 0.152 $\mathrm{mmol}, 60 \%$ ). Polymer 3 remaining in the cellulose thimble was removed and dried under high vacuum for 16 hours to give THF insoluble polymer 3 (0.071g, $0.102 \mathrm{mmol}, 40 \%)$. 


\section{Post-Polymerization Modification Procedures:}

\section{Thiol capping of polymer 3, 4:}

Polymer $3(0.80 \mathrm{~g}, 1.2 \mathrm{mmol}$, repeat unit eq) was swollen in dioxane (8 $\mathrm{mL})$. Butanethiol $(1.05 \mathrm{~g}$, $11.2 \mathrm{mmol}, 10 \mathrm{eq})$ was added, followed by AIBN $(0.095 \mathrm{~g}, 0.58 \mathrm{mmol}, 0.50 \mathrm{eq})$. The reaction mixture was then heated to $90{ }^{\circ} \mathrm{C}$ for fourteen hours. The following morning the mixture was precipitated from ice water $(100 \mathrm{~mL})$. The precipitate was then washed with cold methanol $(2 \mathrm{x}$ $100 \mathrm{~mL}$ ). The precipitate was centrifuged at $2500 \times \mathrm{g}$ for 5 minutes, and the resulting pellet was dried with high vacuum at $60{ }^{\circ} \mathrm{C}$ to yield an orange solid $(0.712 \mathrm{~g}, 1.03 \mathrm{mmol}, 89 \%)$. ${ }^{1} \mathrm{H}$ NMR $\left(\mathrm{CDCl}_{3}, 500 \mathrm{MHz}\right), \delta \mathrm{ppm}: 6.33(\mathrm{t}, J=14.4 \mathrm{~Hz}, 1.5 \mathrm{H}), 6.24(\mathrm{t}, J=13.0 \mathrm{~Hz}, 0.5 \mathrm{H}), 2.65(\mathrm{~m}, 4 \mathrm{H})$, $1.59(\mathrm{~m}, 4 \mathrm{H}), 1.45-1.27(\mathrm{~m}, 4 \mathrm{H}) .{ }^{19} \mathrm{~F} \mathrm{NMR}\left(\mathrm{CDCl}_{3}, 376 \mathrm{MHz}\right), \delta \mathrm{ppm}:-105.35(\mathrm{~s}, 3 \mathrm{~F}),-108.53$ (s, 1F), -121.52 (s, 4F), -122.91 (s, 1F), -123.27(s, 3F). FT-IR: 2932 (C-H str) (w), 1635 (C=C str) (s), 1100-1200 (C-F bend) (vs).

\section{Sonogashira coupling of polymer 4, 5:}

Polymer $4(0.100 \mathrm{~g}, 0.145 \mathrm{mmol}$, repeat unit eq) was swollen in diisopropylamine (1 $\mathrm{mL}) .1$ Octyne $(0.095 \mathrm{~g}, 0.87 \mathrm{mmol}, 6.0 \mathrm{eq})$ was added. The solution was degassed via freeze-pumpthaw $(x 3)$. Copper iodide $(0.0015 \mathrm{~g}, 0.0070 \mathrm{mmol}, 0.05 \mathrm{eq})$ and tetrakis(triphenylphosphine) palladium $(0.008 \mathrm{~g}, 0.007 \mathrm{mmol}, 0.05 \mathrm{eq})$ were added. The reaction mixture was then heated to $50{ }^{\circ} \mathrm{C}$ for fourteen hours. The following morning the mixture was precipitated from saturated ammonium chloride $(50 \mathrm{~mL})$, followed by washing with water $(50 \mathrm{~mL})$ and methanol $(50 \mathrm{~mL})$. The precipitate was centrifuged at $2500 \times \mathrm{g}$ for 5 minutes, and the resulting pellet was dried under high vacuum to yield a light brown solid $(0.048 \mathrm{~g}, 0.074 \mathrm{mmol}, 74 \%) .{ }^{1} \mathrm{H} \mathrm{NMR}\left(\mathrm{CDCl}_{3}\right.$, $500 \mathrm{MHz}), \delta$ ppm: $5.71(\mathrm{t}, J=16.2 \mathrm{~Hz}, 1.4 \mathrm{H}), 5.57(\mathrm{t}, J=11.3 \mathrm{~Hz}, 0.7 \mathrm{H}), 2.40-2.27(\mathrm{~m}, 5.4 \mathrm{H})$, $2.27-2.18(\mathrm{~m}, 1.3 \mathrm{H}), 1.64-1.47(\mathrm{~m}, 10 \mathrm{H}), 1.47-1.18(\mathrm{~m}, 16 \mathrm{H}), 0.89(\mathrm{t}, J=6.7 \mathrm{~Hz}, 6 \mathrm{H}) .{ }^{19} \mathrm{~F}$ NMR ( $\left.\mathrm{CDCl}_{3}, 376 \mathrm{MHz}\right), \delta$ ppm: -105.47 (m, 3F), -108.31 (m, 1F), -121.93 (m, 4F), -123.81 (m, 4F). FT-IR: 2932 (C-H str) (w), 2222 (C三C str) (w), 1630 (C=C str) (s), 1100-1200 (C-F bend) (vs). TGA: $10 \%$ mass loss at $245^{\circ} \mathrm{C} . T_{g}(\mathrm{DSC}):-25.0^{\circ} \mathrm{C}$

\section{Stille coupling of polymer 4,6 :}

Polymer $4(0.100 \mathrm{~g}, 0.145 \mathrm{mmol}$, repeat unit eq) was swollen in toluene (1 $\mathrm{mL})$. 2-(tributytin) furan $(0.30 \mathrm{~g}, 0.87 \mathrm{mmol}, 6.0 \mathrm{eq})$ was added. The solution was degassed via freeze-pump-thaw (x3). Tetrakis(triphenylphosphine) palladium $(0.008 \mathrm{~g}, 0.007 \mathrm{mmol}, 0.05 \mathrm{eq})$ was then added and capped. The reaction mixture was heated to $60{ }^{\circ} \mathrm{C}$ for fourteen hours. The following morning the mixture was precipitated from cold methanol $(50 \mathrm{~mL})$, followed by washing with methanol two times $(50 \mathrm{~mL})$. The precipitate was centrifuged at $2500 \mathrm{Xg}$ for 5 minutes, and the resulting pellet was dried with high vacuum to yield a light orange solid $(0.046 \mathrm{~g}, 0.082 \mathrm{mmol}$, 57\%). ${ }^{1} \mathrm{H} \mathrm{NMR}\left(\mathrm{CDCl}_{3}, 500 \mathrm{MHz}\right), \delta \mathrm{ppm}: 7.45(\mathrm{~s}, 0.6 \mathrm{H}), 7.41(\mathrm{~s}, 1.4 \mathrm{H}), 6.66(\mathrm{~s}, 0.6 \mathrm{H}), 6.53(\mathrm{~s}$, $1.4 \mathrm{H}), 6.42(\mathrm{~s}, 2 \mathrm{H}), 6.04(\mathrm{t}, J=16.2 \mathrm{~Hz}, 1.4 \mathrm{H}), 5.46(\mathrm{t}, J=16.2 \mathrm{~Hz}, 0.6 \mathrm{H}), 2.61-2.44(\mathrm{~m}, 4 \mathrm{H})$, $1.66-1.52(\mathrm{~m}, 2 \mathrm{H}), 1.49-1.27(\mathrm{~m}, 6 \mathrm{H}) .{ }^{19} \mathrm{~F} \mathrm{NMR}\left(\mathrm{CDCl}_{3}, 376 \mathrm{MHz}\right), \delta \mathrm{ppm}:-104.52(\mathrm{~s}, 3 \mathrm{~F})$, 104.87 (s, 1F), -121.39 (s, 4F), -122.54 - -124.29 (m, 4F). FT-IR: 2932 (C-H str) (w), 1707 (C=C str) furan (s), 1635 ( $C=\mathrm{C}$ str) alkene (s), 1100-1200 (C-F bend) (vs). TGA: 10\% mass loss at $266^{\circ} \mathrm{C} . T_{g}$ (DSC): $17.0^{\circ} \mathrm{C}$ 


\section{Suzuki coupling of polymer 4,7 :}

Polymer 4 (0.100 g, $0.145 \mathrm{mmol}$, repeat unit eq) was swollen in dimethylformamide (2 mL). 7heptenyl boronic acid $(0.082 \mathrm{~g}, 0.58 \mathrm{mmol}, 4.0 \mathrm{eq})$ was added. The solution was degassed via freeze-pump-thaw (x3). Potassium carbonate $(0.12 \mathrm{~g}, 0.87 \mathrm{mmol}, 6.0 \mathrm{eq})$ and tetrakis(triphenylphosphine) palladium $(0.008 \mathrm{~g}, 0.007 \mathrm{mmol}, 0.05 \mathrm{eq})$ were then added. The reaction mixture was heated to $85{ }^{\circ} \mathrm{C}$ for fourteen hours. The following morning the mixture was precipitated from saturated sodium bicarbonate $(50 \mathrm{~mL})$, followed by washing with water (50 $\mathrm{mL}$ ) and methanol $(2 \times 50 \mathrm{~mL})$. The precipitate was centrifuged at $2500 \times g$ for 5 minutes, and the resulting pellet was dried under high vacuum to yield a light brown solid $(0.066 \mathrm{~g}, 0.11$ mmol, $74 \%)$ that included $93 \%$ alkene addition and $7 \%$ iodide elimination. ${ }^{1} \mathrm{H} N M R\left(\mathrm{CDCl}_{3}, 500\right.$ $\mathrm{MHz}), \delta \mathrm{ppm:} 6.50(\mathrm{~d}, J=16.2 \mathrm{~Hz}, 1 \mathrm{H}), 5.96(\mathrm{~s}, 3 \mathrm{H}), 5.41-5.30(\mathrm{~m}, 1 \mathrm{H}), 5.29-5.20(\mathrm{~m}, 1 \mathrm{H})$, $2.52-2.03(\mathrm{~m}, 8 \mathrm{H}), 1.65-1.15(\mathrm{~m}, 20 \mathrm{H}), 0.89(\mathrm{~s}, 6 \mathrm{H}) .{ }^{19} \mathrm{~F} \mathrm{NMR}\left(\mathrm{CDCl}_{3}, 376 \mathrm{MHz}\right), \delta \mathrm{ppm}:-$ 102.92 (s, 1F), -104.38 (s, 3F), -121.44 (s, 4F), -123.01 - -123.80 (m, 4F). FT-IR: 2932 (C-H str) (w), 1646 ( $\mathrm{C}=\mathrm{C}$ str) diene (s), 1100-1200 (C-F bend) (vs). TGA: $10 \%$ mass loss at $333^{\circ} \mathrm{C} . T_{g}$ (DSC): $-37^{\circ} \mathrm{C}$

\section{Phenol coupling of polymer 4, 8:}

Polymer 4 (0.100 g, $0.145 \mathrm{mmol}$, repeat unit eq) was swollen in dioxane $(1 \mathrm{~mL})$. Phenol $(0.110$ $\mathrm{g}, 1.16 \mathrm{mmol}, 8.00 \mathrm{eq})$ and dimethyl glycine $(0.009 \mathrm{~g}, 0.09 \mathrm{mmol}, 0.6 \mathrm{eq})$ were added. The solution was degassed via freeze-pump-thaw (x3). Cesium carbonate $(0.204 \mathrm{~g}, 0.580 \mathrm{mmol}$, $4.00 \mathrm{eq})$ and copper iodide $(0.005 \mathrm{~g}, 0.03 \mathrm{mmol}, 0.2 \mathrm{eq})$ were then added and the reaction was set to $90{ }^{\circ} \mathrm{C}$ for 16 hours. The following morning the mixture was precipitated from saturated sodium bicarbonate $(50 \mathrm{~mL})$, followed by washing with water $(50 \mathrm{~mL})$ and methanol $(2 \times 50 \mathrm{~mL})$. The precipitate was centrifuged at $2500 \times \mathrm{g}$ for 5 minutes, and the resulting pellet was dried under high vacuum to yield a light yellow solid $(0.069 \mathrm{~g}, 0.10 \mathrm{mmol}, 88 \%)$ that included $65 \%$ phenol addition and 35\% en-yne rearrangement. ${ }^{1} \mathrm{H}$ NMR $\left(\mathrm{CDCl}_{3}, 500 \mathrm{MHz}\right), \delta \mathrm{ppm}: 7.37$ (s, 2.6H), $7.20(\mathrm{~s}, 1.3 \mathrm{H}), 6.98(\mathrm{~s}, 2.6 \mathrm{H}), 5.67(\mathrm{~d}, J=38.6 \mathrm{~Hz}, 0.7 \mathrm{H}), 4.43(\mathrm{t}, J=15.2 \mathrm{~Hz}, 1.3 \mathrm{H}), 2.52$ $(\mathrm{s}, 2.6 \mathrm{H}), 2.42(\mathrm{~s}, 1.3), 1.74(\mathrm{~s}, 2.6 \mathrm{H}), 1.62(\mathrm{~s}, 1.3), 1.57(\mathrm{~s}, 1.3 \mathrm{H}), 1.47(\mathrm{~s}, 2.6 \mathrm{H}) .{ }^{19} \mathrm{~F} \mathrm{NMR}$ $\left(\mathrm{CDCl}_{3}, 376 \mathrm{MHz}\right), \delta \mathrm{ppm}:-102.62(\mathrm{~s}, 2.75 \mathrm{~F}),-117.95$ (1.85F), -121.55 (s, 2.75F), -122.99 (s, 1.25F), -123.51 (4F). FT-IR: 2932 (C-H str) (w), 2230 (C三C str) (w), 1660 (C=C str) alkene (s), 1590 ( $\mathrm{C}=\mathrm{C}$ str) aromatic (s), 1490 ( $\mathrm{C}=\mathrm{C}$ bend) aromatic (s), 1100-1200 (C-F bend) (vs). TGA: $10 \%$ mass loss at $329^{\circ} \mathrm{C}$. $T_{g}$ (DSC): $-10^{\circ} \mathrm{C}$.

\section{Thiophenol coupling of polymer 4, 9:}

Polymer $4(0.100 \mathrm{~g}, 0.145 \mathrm{mmol}$, repeat unit eq) was swollen in toluene $(2 \mathrm{~mL})$. Thiophenol $(0.127 \mathrm{~g}, 1.16 \mathrm{mmol}, 8.00 \mathrm{eq}), 1,10-$ phenanthroline $(0.010 \mathrm{~g}, 0.056 \mathrm{mmol}, 0.40 \mathrm{eq})$, and triphenylphosphine $(0.030 \mathrm{~g}, 0.11 \mathrm{mmol}, 0.80 \mathrm{eq})$ were added. The solution was degassed via freeze-pump-thaw (x3). Potassium phosphate $(0.121 \mathrm{~g}, 0.560 \mathrm{mmol}, 4.00 \mathrm{eq})$ and copper iodide $(0.010 \mathrm{~g}, 0.058 \mathrm{mmol}, 0.4 \mathrm{eq})$ were then added and the reaction was set to $90{ }^{\circ} \mathrm{C}$ for 16 hours. The following morning the mixture was precipitated from saturated sodium bicarbonate $(50 \mathrm{~mL})$, followed by washing with water $(50 \mathrm{~mL})$ and methanol $(2 \times 50 \mathrm{~mL})$. The precipitate was centrifuged at $2500 \times \mathrm{g}$ for 5 minutes, and the resulting pellet was dried under high vacuum to yield a light orange solid $(0.065 \mathrm{~g}, 0.10 \mathrm{mmol}, 79 \%)$. ${ }^{1} \mathrm{H} \mathrm{NMR}\left(\mathrm{CDCl}_{3}, 500 \mathrm{MHz}\right), \delta \mathrm{ppm}: 7.53-$ $7.31(\mathrm{~m}, 10 \mathrm{H}), 5.73-5.47(\mathrm{~m}, 0.5 \mathrm{H}), 4.95-4.72(\mathrm{~m}, 1.5 \mathrm{H}), 2.56-2.36(\mathrm{~m}, 3 \mathrm{H}), 2.07(\mathrm{~m}, 1 \mathrm{H})$, $1.78-1.26(\mathrm{~m}, 8 \mathrm{H}) .{ }^{19} \mathrm{~F} \mathrm{NMR}\left(\mathrm{CDCl}_{3}, 376 \mathrm{MHz}\right), \delta \mathrm{ppm}: \quad-103.75(\mathrm{~s}, 3 \mathrm{~F}),-104.44(\mathrm{~s}, 1 \mathrm{~F})$, 121.57 (s, 4F), -123.02 (s, 1F), -123.55 (s, 3F). FT-IR: 2932 (C-H str) (w), 1626 (C=C str) alkene 
(s), 1583 (C=C str) aromatic (s), 1477 (C=C bend) aromatic (s), 1100-1200 (C-F bend) (vs). TGA: $10 \%$ mass loss at $370{ }^{\circ} \mathrm{C}$. $T_{g}$ (DSC): $-6{ }^{\circ} \mathrm{C}$.

\section{Kumada coupling of polymer 3, 10:}

Polymer 3 (0.200 g, $0.290 \mathrm{mmol}$, repeat unit eq) was swollen in THF (4 mL). Iron (III) acetylacetonate $(0.050 \mathrm{~g}, 0.15 \mathrm{mmol}, 0.50 \mathrm{eq})$ was added under nitrogen. Methyl magnesium bromide $(1 \mathrm{M})(1.8 \mathrm{~mL}, 1.8 \mathrm{mmol}, 6.0 \mathrm{eq})$ was then added dropwise over an hour at room temperature. After complete addition of methyl magnesium bromide, methanol $(1 \mathrm{~mL})$ was slowly added to quench the remaining reagents. The solution was precipitated from water (50 $\mathrm{mL})$, followed by washing with methanol $(2 \times 50 \mathrm{~mL})$. The precipitate was centrifuged at $2500 \times$ $g$ for 5 minutes, and the resulting pellet was dried under high vacuum to yield a brown solid $(0.115 \mathrm{~g}, 0.220 \mathrm{mmol}, 87 \%)$ as $70 \%$ methyl addition and $30 \%$ iodide reduction by proton NMR. ${ }^{1} \mathrm{H}$ NMR $\left(\mathrm{CDCl}_{3}, 500 \mathrm{MHz}\right), \delta \mathrm{ppm}: 6.44-6.30(\mathrm{~m}, 0.25 \mathrm{H}), 6.12-6.00(\mathrm{~m}, 0.25 \mathrm{H}), 5.68-5.54$ $(\mathrm{m}, 0.25 \mathrm{H}), 5.54-5.45(\mathrm{~m}, 0.25 \mathrm{H}), 5.31(\mathrm{t}, J=16.7 \mathrm{~Hz}, 1.5 \mathrm{H}), 2.24(\mathrm{~s}, 3 \mathrm{H}), 2.19-2.03(\mathrm{~m}$, $1 \mathrm{H}), 1.87(\mathrm{~s}, 4.5 \mathrm{H}), 1.55(\mathrm{~s}, 1 \mathrm{H}), 1.45(\mathrm{~s}, 3 \mathrm{H}), 1.38-1.11(\mathrm{~m}, 4 \mathrm{H}) .{ }^{19} \mathrm{~F} \mathrm{NMR}\left(\mathrm{CDCl}_{3}, 376 \mathrm{MHz}\right)$, ठ ppm: $\quad-104.76(\mathrm{~s}, 3 \mathrm{~F}),-105.37(\mathrm{~s}, 0.35 \mathrm{~F}),-106.65(\mathrm{~s}, 0.17 \mathrm{~F}),-111.27$ (s, 0.37F), -121.58 (s, 4F), -123.23 - -124.66 (m, 4F). FT-IR: 2932 (C-H str) (w), 1667 (C=C str) (s), 1100-1200 (C-F bend) (vs). TGA: $10 \%$ mass loss at $373^{\circ} \mathrm{C} . T_{g}$ (DSC): $-39^{\circ} \mathrm{C}$

Elimination of iodide from polymer 3, 11:

Polymer $4(0.200 \mathrm{~g}, 0.290 \mathrm{mmol}$, repeat unit eq) was swollen in toluene $(4 \mathrm{~mL})$. Tetramethylguanidine $(0.33 \mathrm{~g}, 2.9 \mathrm{mmol}, 10 \mathrm{eq})$ was added dropwise and the solution was allowed to stir for 16 hours. The following morning the reaction mixture was precipitated from cold methanol $(50 \mathrm{~mL})$ and additionally washed with saturated ammonium chloride $(50 \mathrm{~mL})$, followed by an additional wash of methanol $(50 \mathrm{~mL})$. The precipitate was centrifuged at $2500 \mathrm{X}$ $g$ for 5 minutes, and the resulting pellet was dried under high vacuum to yield a brown solid $(0.090 \mathrm{~g}, 0.14 \mathrm{mmol}, 49 \%)$ as polymer with all Z-Isomer iodide eliminated. ${ }^{1} \mathrm{H} \mathrm{NMR}\left(\mathrm{CDCl}_{3}, 500\right.$ $\mathrm{MHz}$ ), $\delta$ ppm: $6.33(\mathrm{t}, J=14.6 \mathrm{~Hz}, 1.5 \mathrm{H}), 2.69-2.53(\mathrm{~m}, 3 \mathrm{H}), 2.36(\mathrm{t}, J=5.5 \mathrm{~Hz}, 1 \mathrm{H}), 1.60(\mathrm{~s}$, $4 \mathrm{H}), 1.47-1.31(\mathrm{~m}, 4 \mathrm{H}) .{ }^{19} \mathrm{~F} \mathrm{NMR}\left(\mathrm{CDCl}_{3}, 376 \mathrm{MHz}\right), \delta \mathrm{ppm}:-96.31(\mathrm{~s}, 1 \mathrm{~F}),-105.37(\mathrm{~s}, 3 \mathrm{~F}),-$ 121.19 (s, 1F), -121.53 (s, 3F), -122.61 (s, 1F), -123.36 (s, 3F). FT-IR: 2932 (C-H str) (w), 2260 (C $\equiv \mathrm{C}$ str) (w), 1635 (C=C str) (s), 1100-1200 (C-F bend) (vs). TGA: $10 \%$ mass loss at $347{ }^{\circ} \mathrm{C} . T_{g}$ (DSC): $-27^{\circ} \mathrm{C}$

\section{Alkyne-azide coupling of polymer 11, 13:}

Polymer 11 (0.010 g, $0.018 \mathrm{mmol}$, repeat unit eq) was dissolved in xylenes $(0.25 \mathrm{~mL})$. 2azidoethyl perfluorooctane $(0.030 \mathrm{~g}, 0.073 \mathrm{mmol}, 4.0 \mathrm{eq})$ was then added and refluxed for 18 hours. The following morning the reaction mixture was precipitated from methanol $(10 \mathrm{~mL})$ and washed with methanol $(2 \times 10 \mathrm{~mL})$. The precipitate was centrifuged at $2500 \times \mathrm{g}$ for 5 minutes, and the resulting pellet was dried under high vacuum to yield a brown solid $(0.013 \mathrm{~g}, 0.014$ mmol, 80\%). ${ }^{1} \mathrm{H}$ NMR $\left(\mathrm{CDCl}_{3}, 500 \mathrm{MHz}\right), \delta \mathrm{ppm}: 6.32(\mathrm{t}, J=15.0 \mathrm{~Hz}, 1 \mathrm{H}), 4.68(\mathrm{~s}, 0.5 \mathrm{H}), 4.53$ $(\mathrm{s}, 0.5 \mathrm{H}), 3.05-2.72(\mathrm{~m}, 2 \mathrm{H}), 2.63(\mathrm{~s}, 3 \mathrm{H}), 1.88-1.30(\mathrm{~m}, 8 \mathrm{H}) .{ }^{19} \mathrm{~F} \mathrm{NMR}\left(\mathrm{CDCl}_{3}, 376 \mathrm{MHz}\right), \delta$ ppm: $\quad-80.89$ (s, 1.5F), -105.37 (s, 3F), -107.42 (s, 0.6F), -109.17 (s, 0.4F), -114.35 (s, 1F), $121.07--122.62(\mathrm{~m}, 7 \mathrm{~F}),-122.88(\mathrm{~s}, 1 \mathrm{~F}),-123.46$ (s, 5F), -126.25 (s, 1F). FT-IR: 2932 (C-H str) (w), 1632 (C=C str) alkene + aromatic (s), 1458 ( $\mathrm{C}=\mathrm{C}$ bend) aromatic 1100-1200 (C-F bend) (vs). 


\section{${ }^{1} \mathrm{H}$ NMR Spectra:}

9,9,10,10,11,11,12,12,13,13,14,14-dodecafluoro-7,16-diiododocosa-7,15-diene, S1, S2:
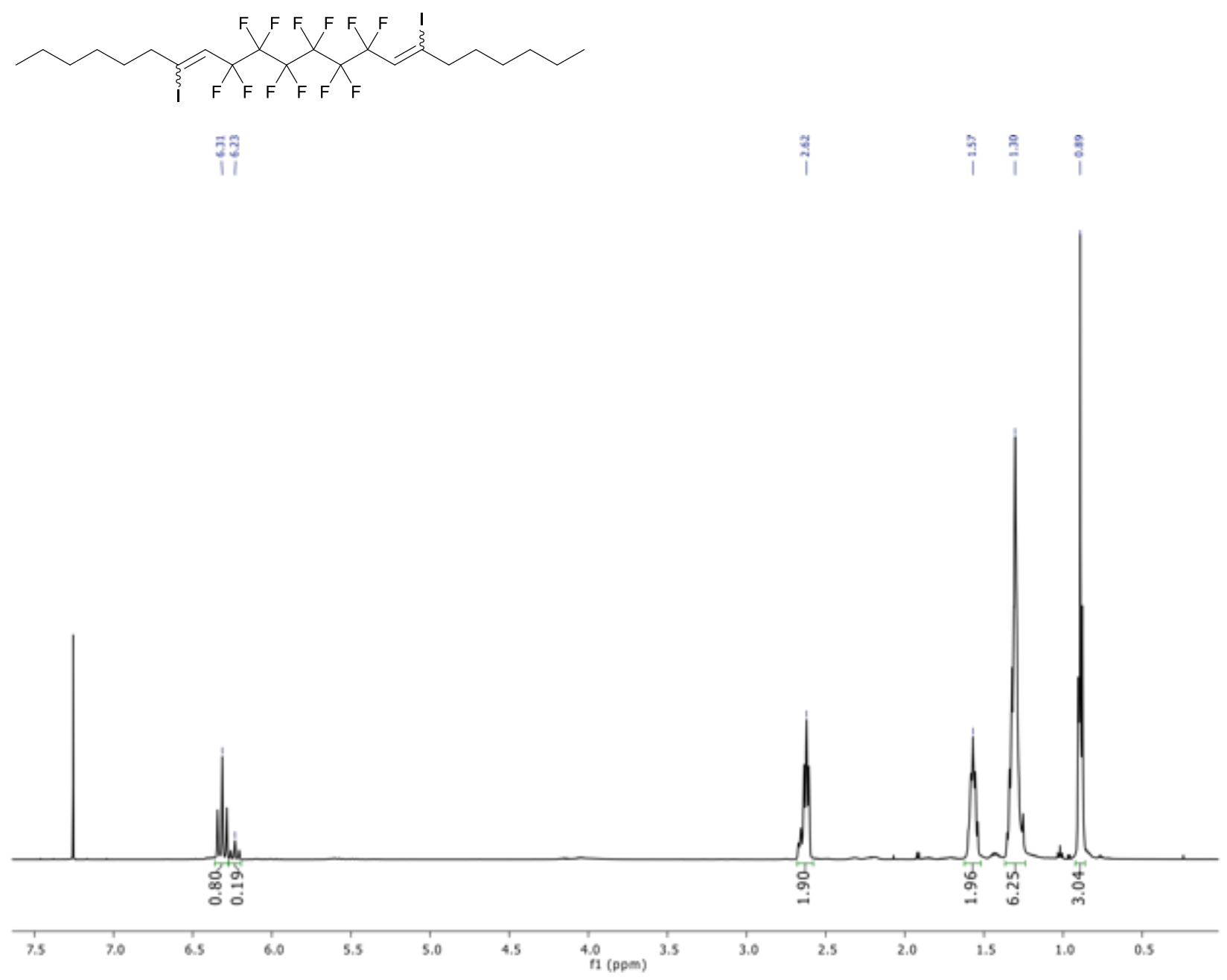
$((9,9,10,10,11,11,12,12,13,13,14,14,14-$ tridecafluorotetradec-7-en-7-yl)oxy)benzene, S4:

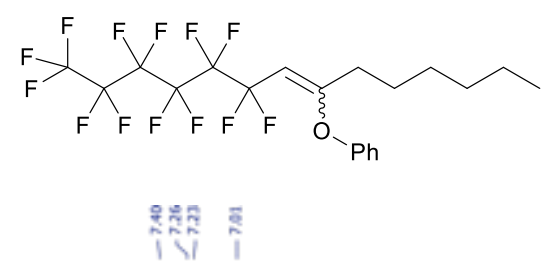

\section{i}
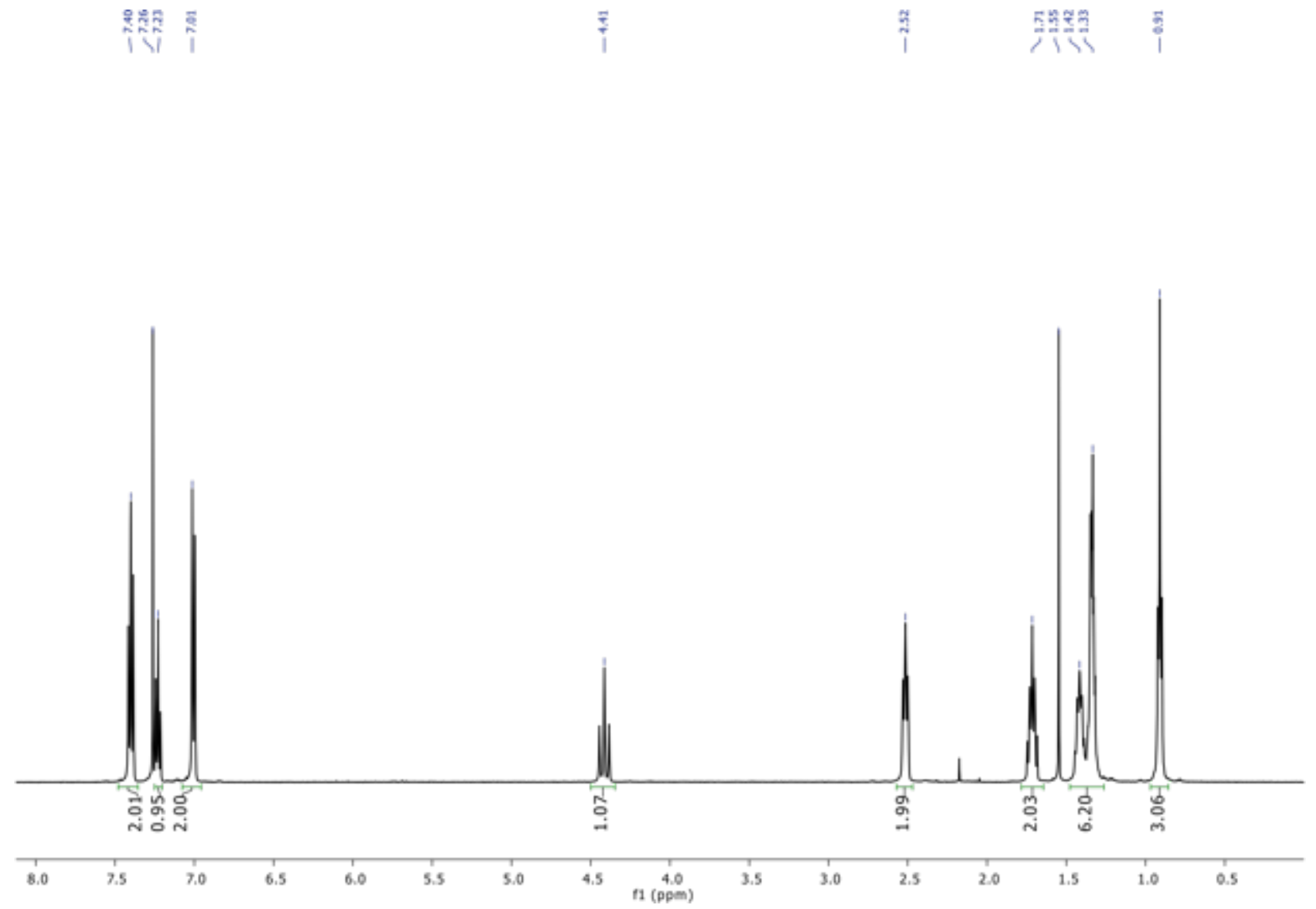
1,1,1,2,2,3,3,4,4,5,5,6-dodecafluorotetradec-6-en-8-yne, S5:

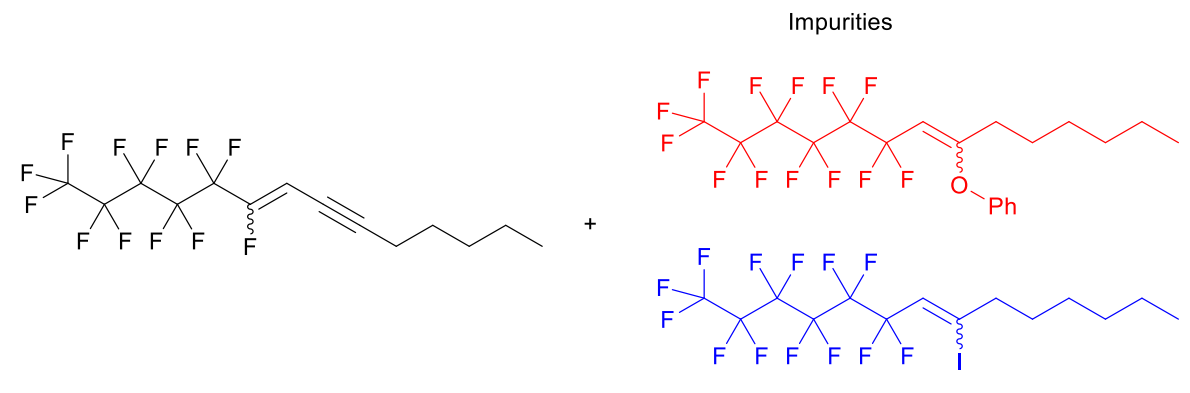

$\underbrace{8}$
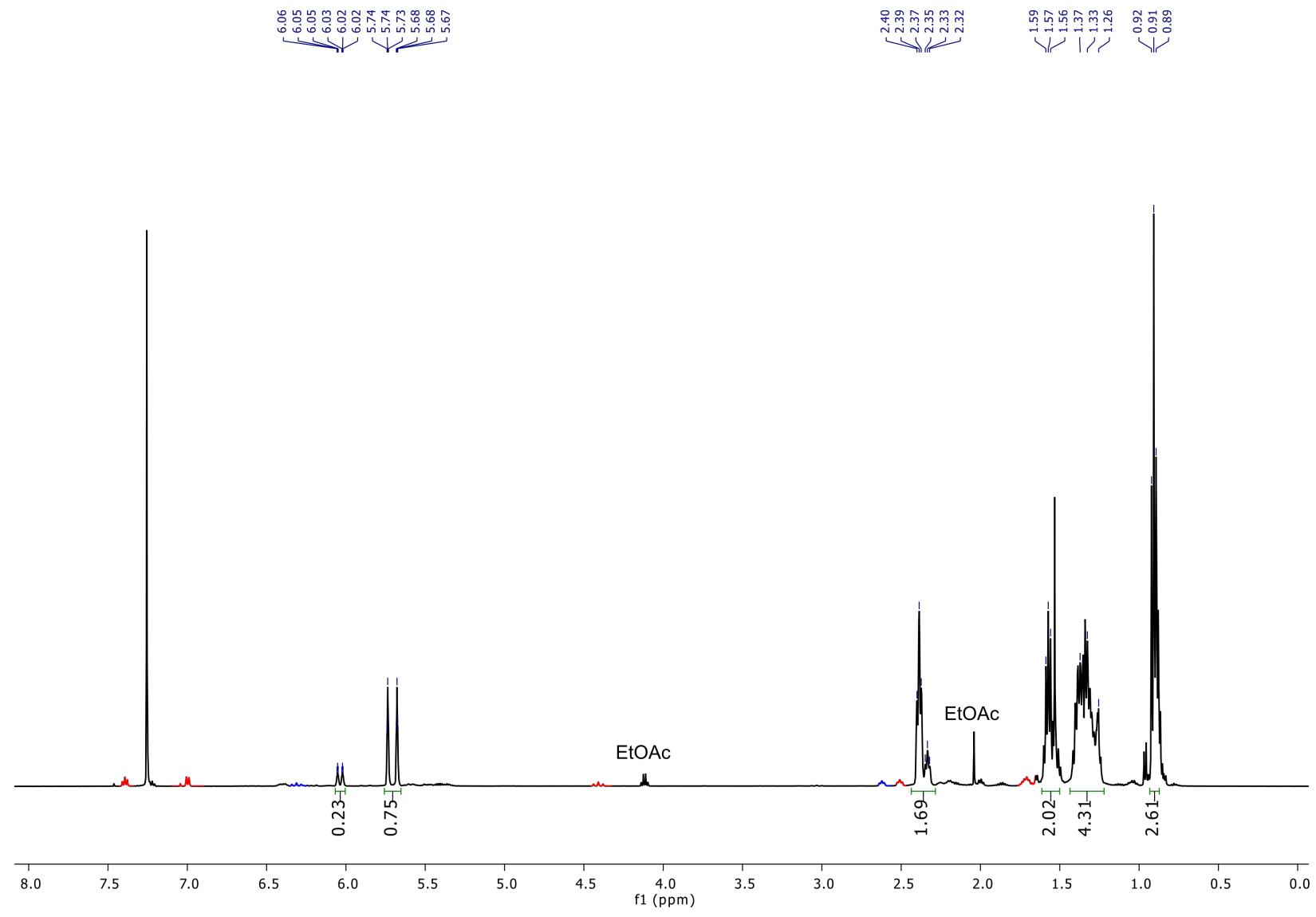
1-perfluorohexyl-2,9-diiodo-1,9-decadiene block polymer, 3:
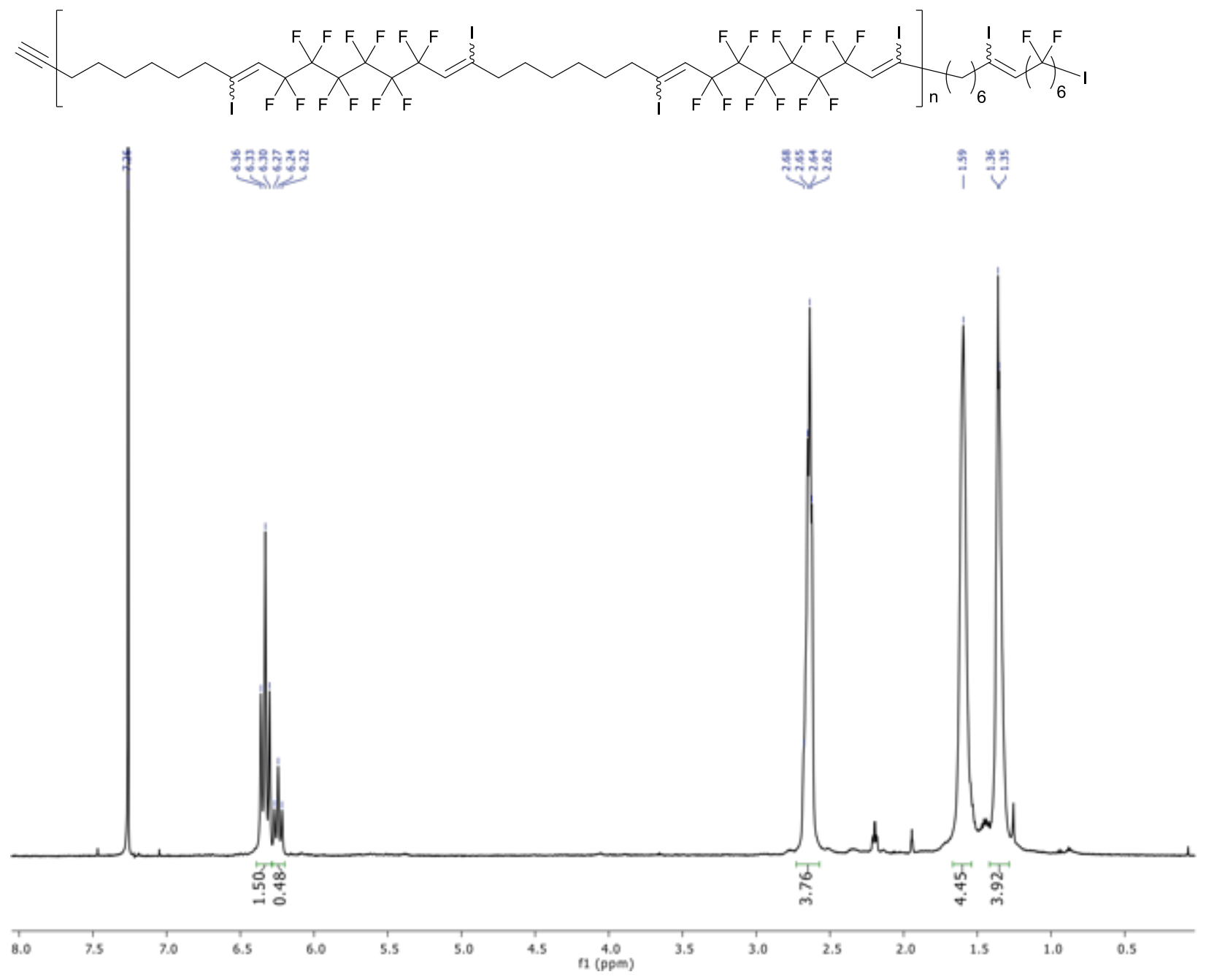
Thiol capping of polymer 3,4 :
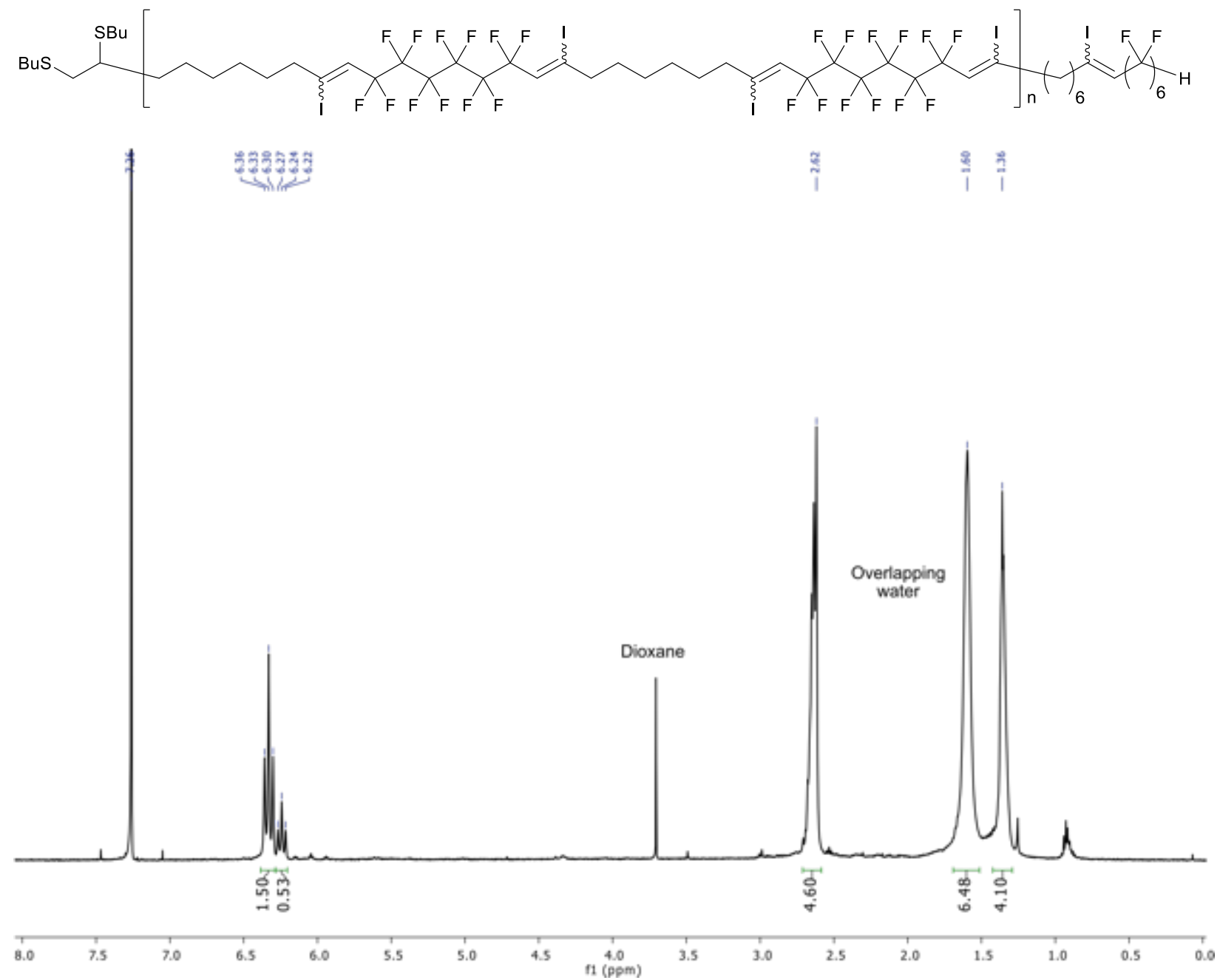
Sonogashira coupling of polymer 4, 5 :
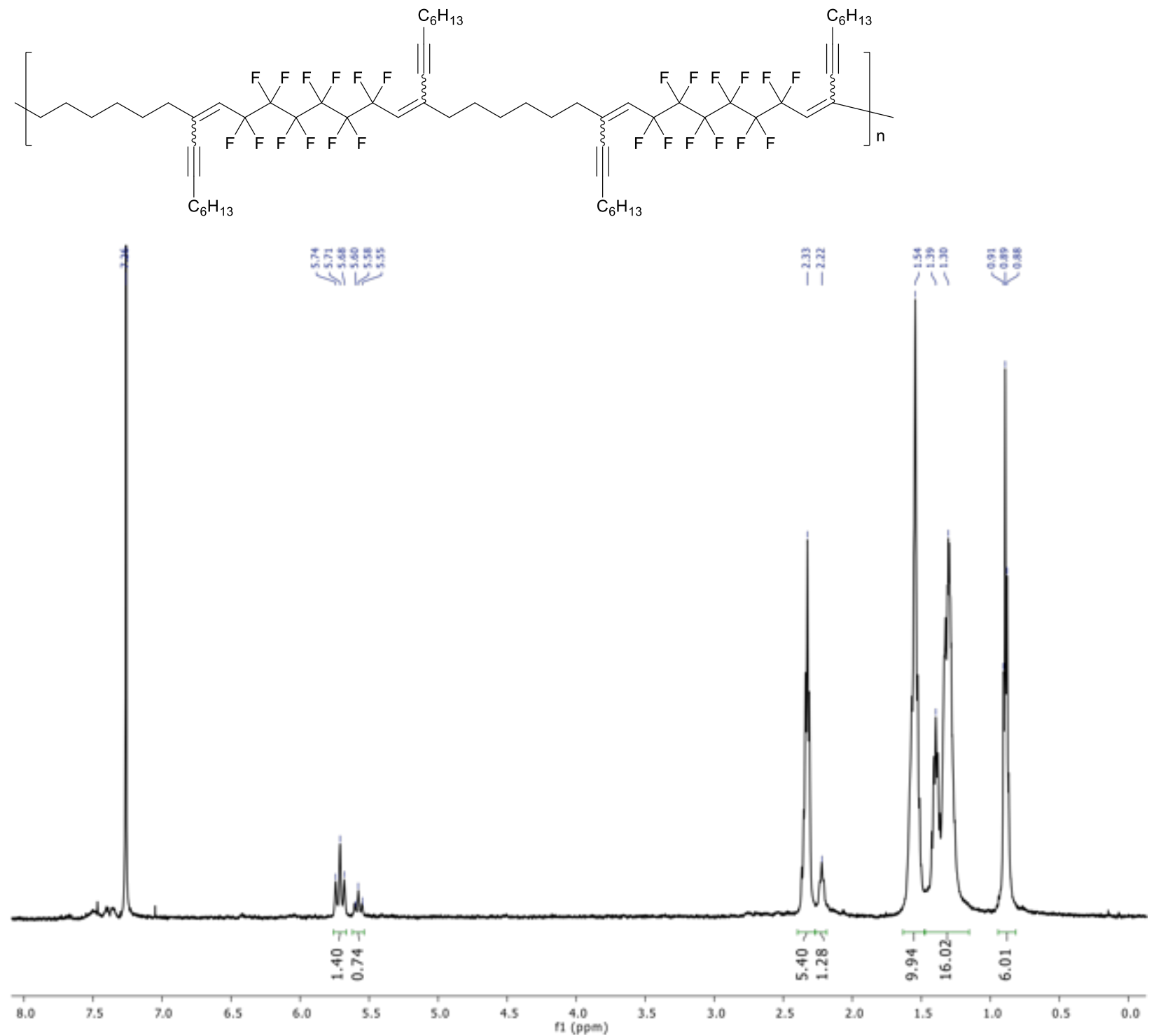
Stille coupling of polymer 4,6 :
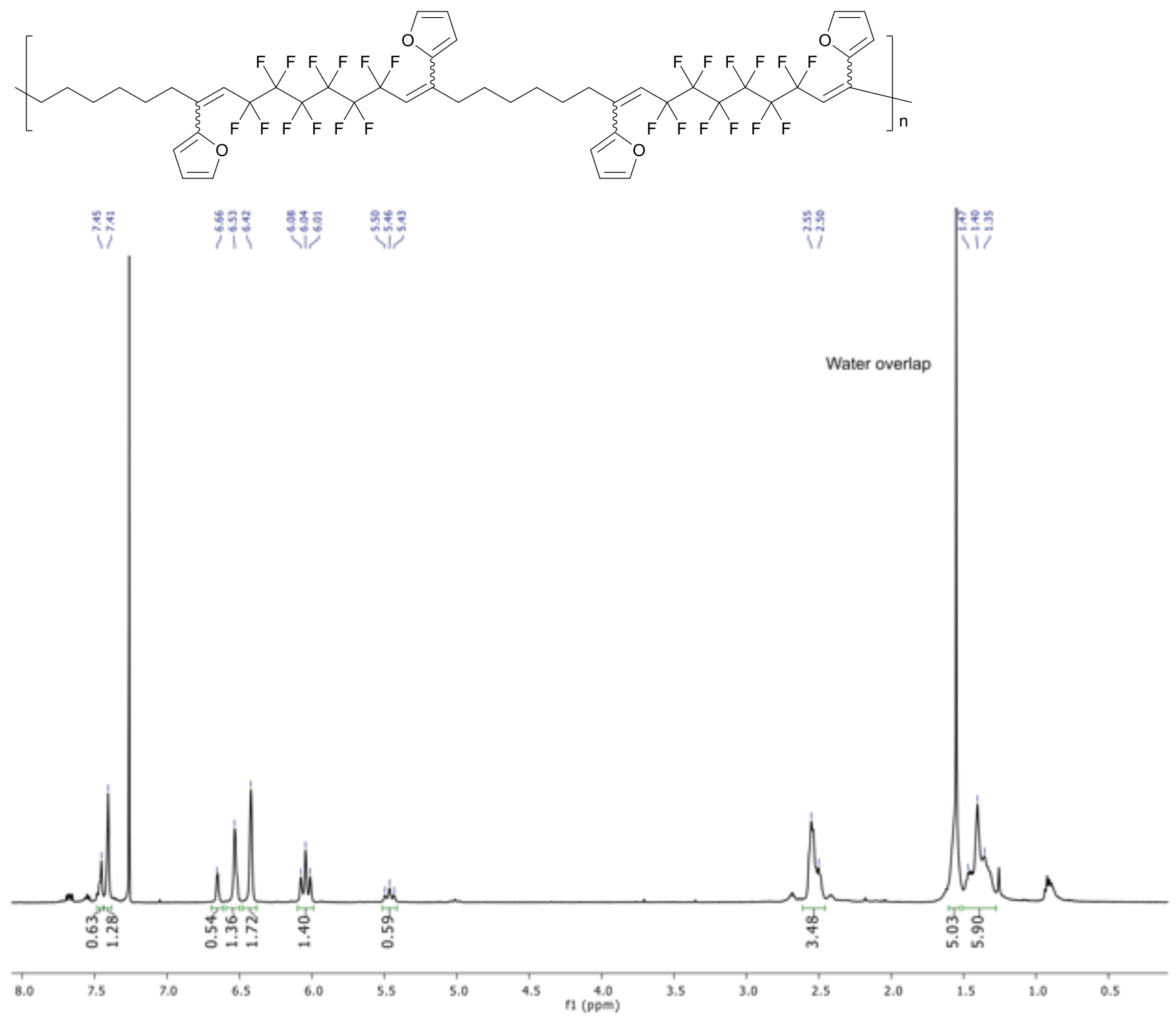
Suzuki coupling of polymer 4,7 :
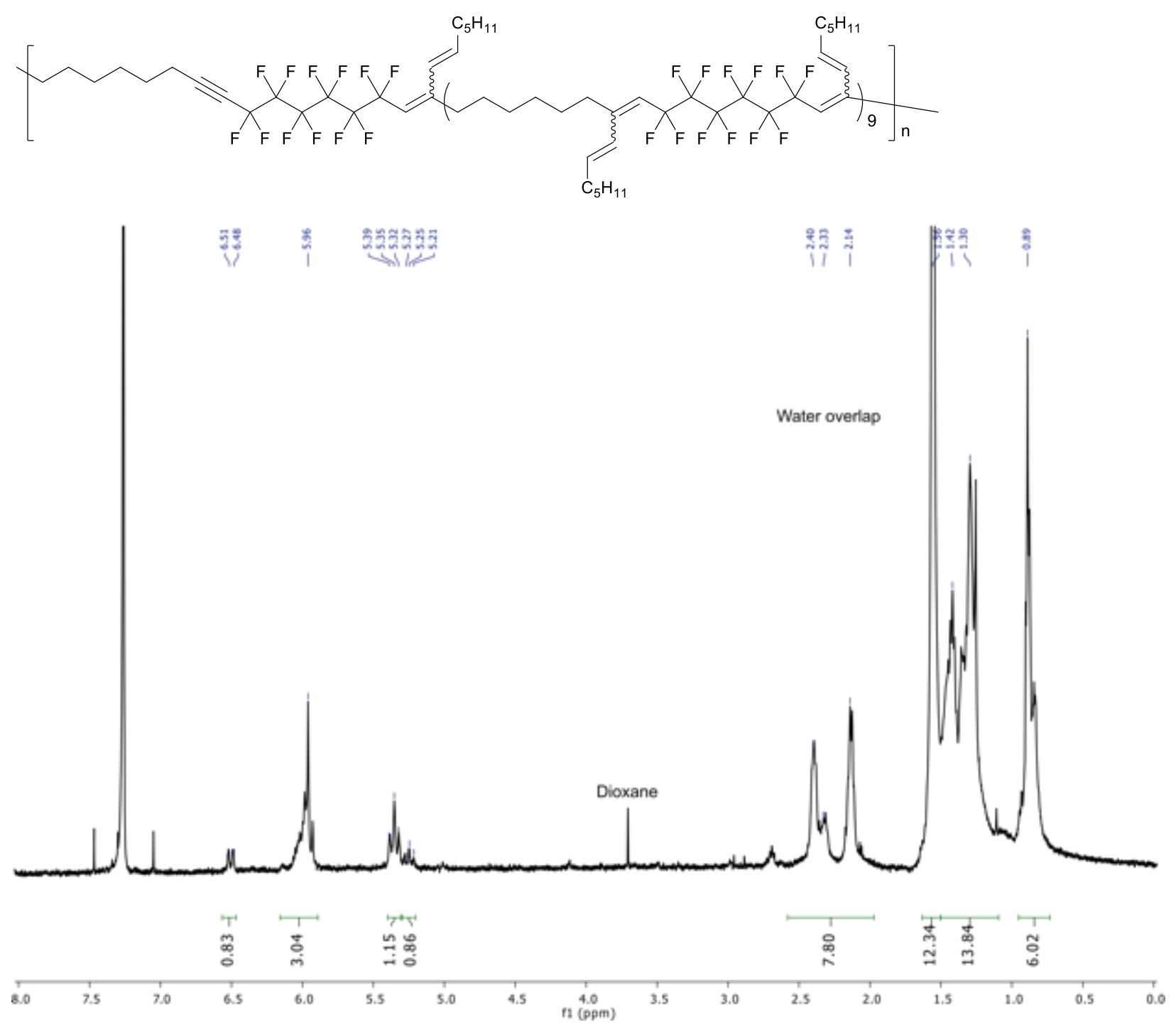
Phenol coupling of polymer 4, 8:

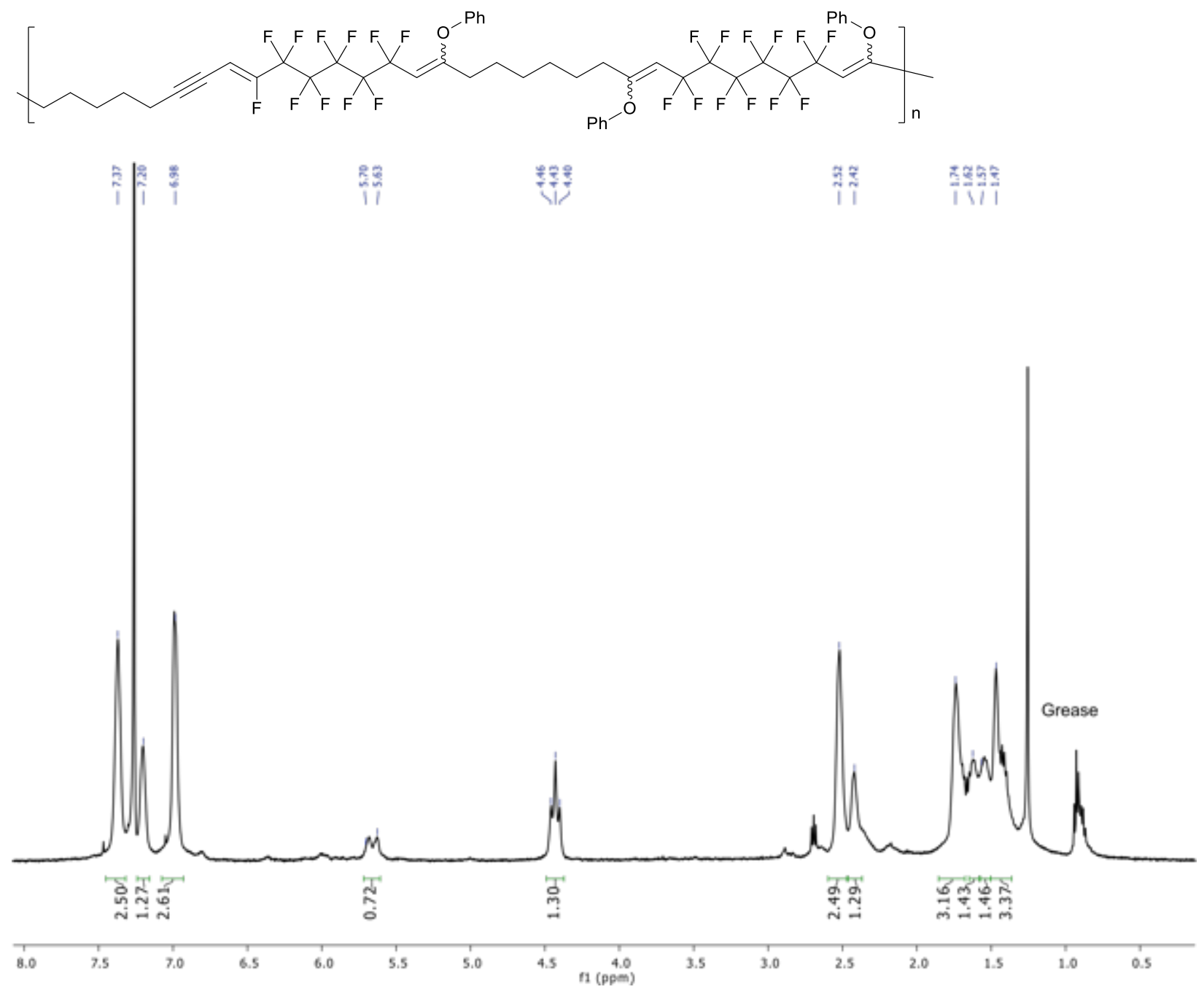


Thiophenol coupling of polymer 4, 9:
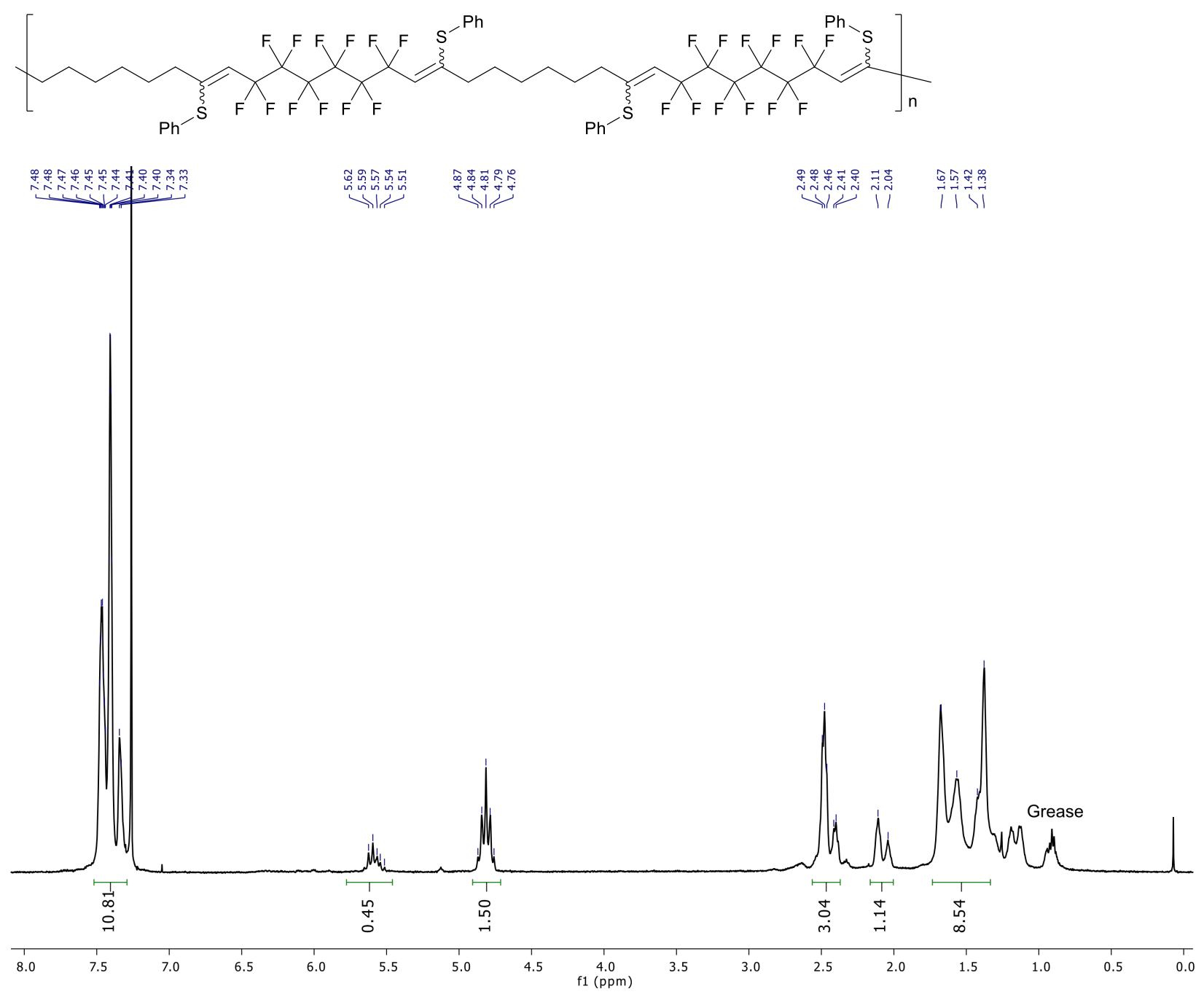
Kumada coupling of polymer 3,10 :
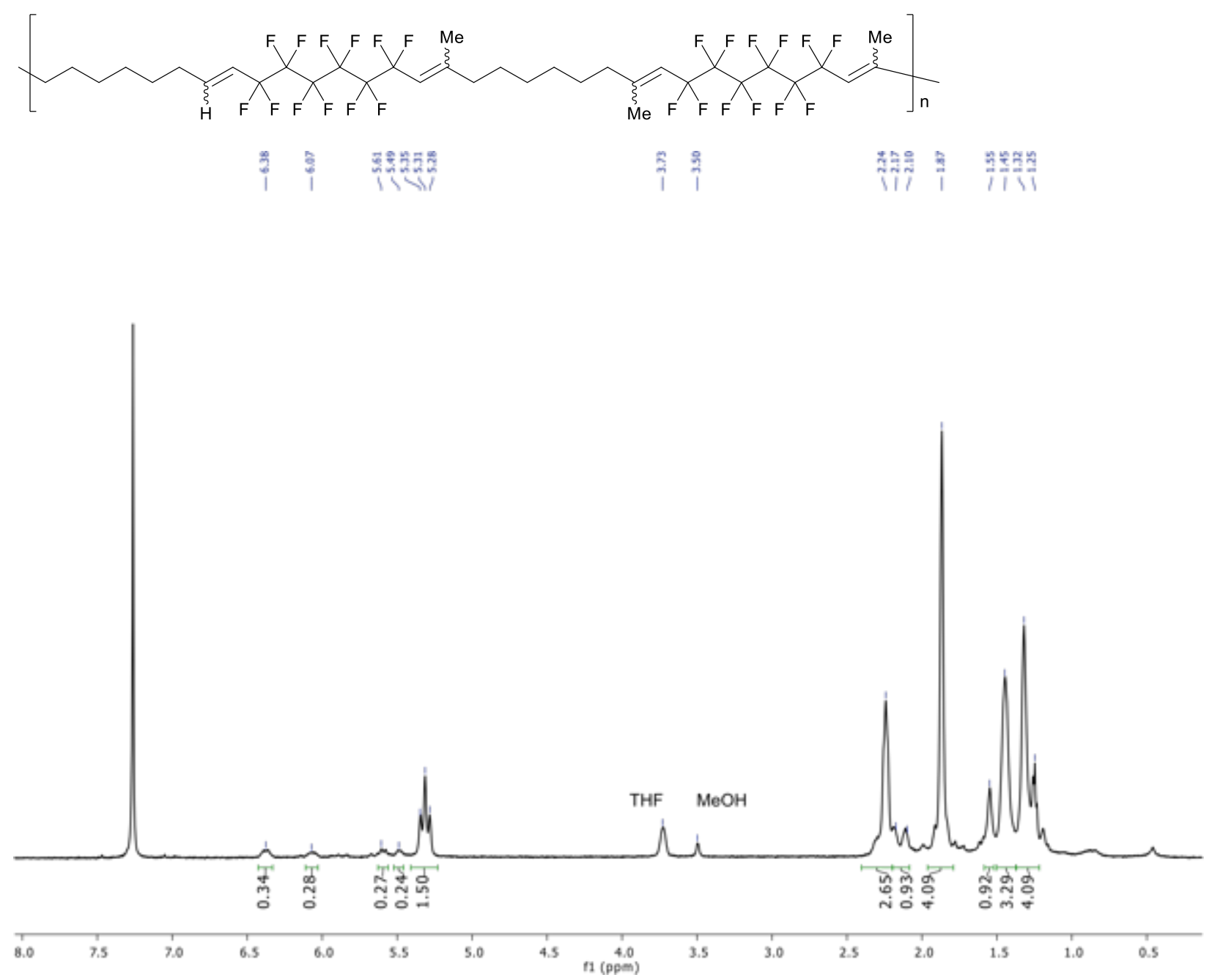
Elimination of iodide from polymer 3,11 :

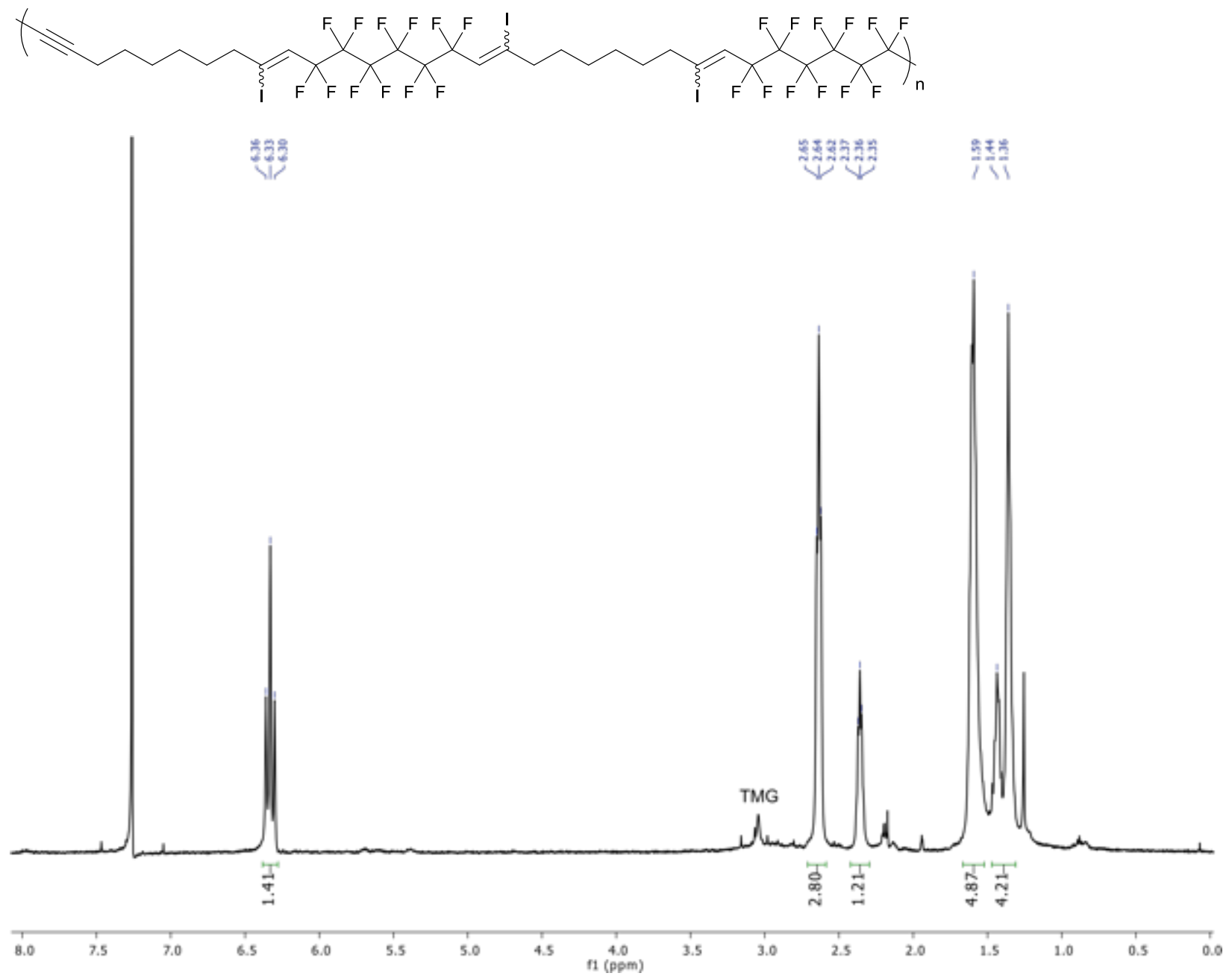


Alkyne-azide coupling of polymer 11, 12:

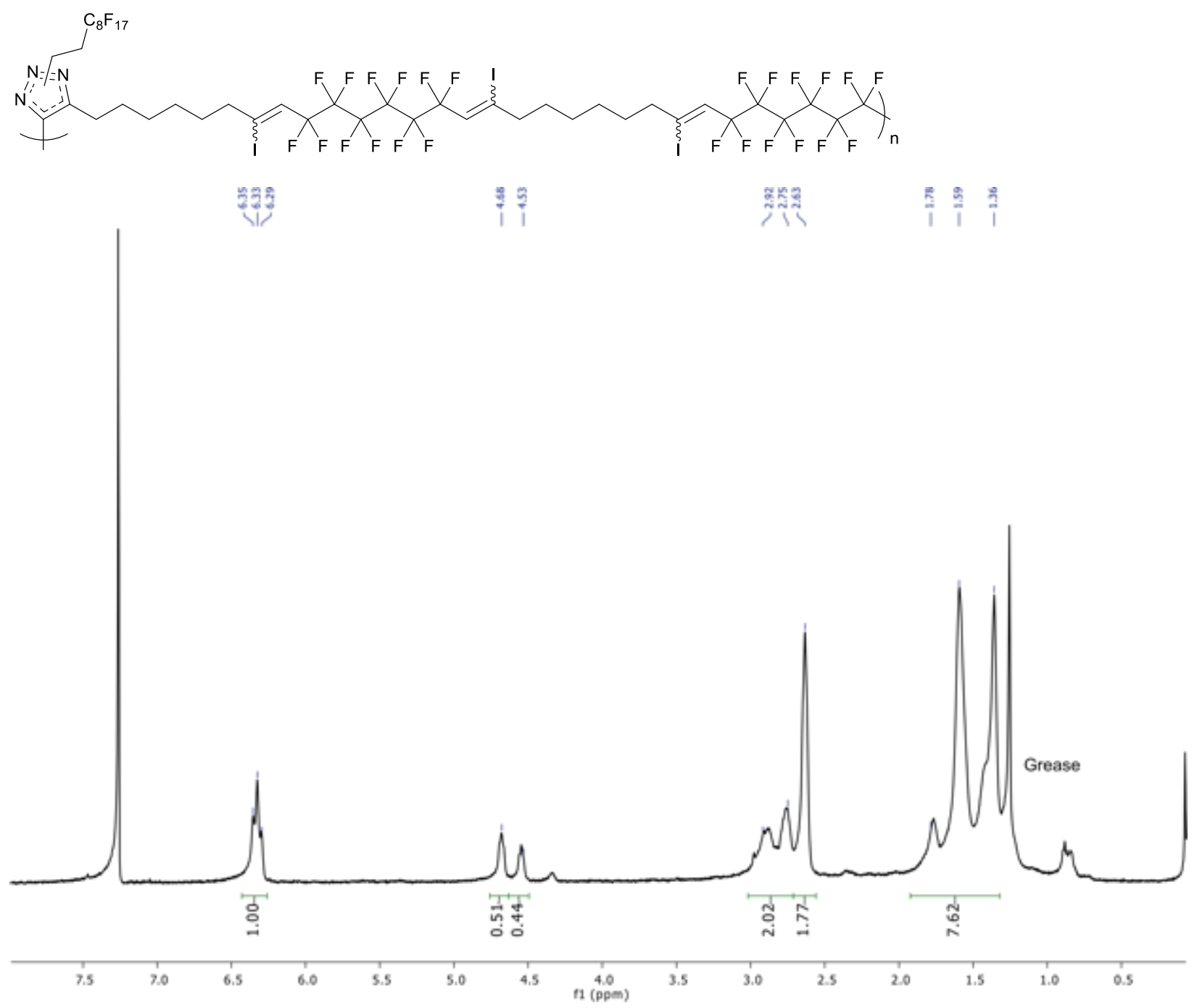


${ }^{13}$ C NMR Spectra:

9,9,10,10,11,11,12,12,13,13,14,14-dodecafluoro-7,16-diiododocosa-7,15-diene, S1, S2:

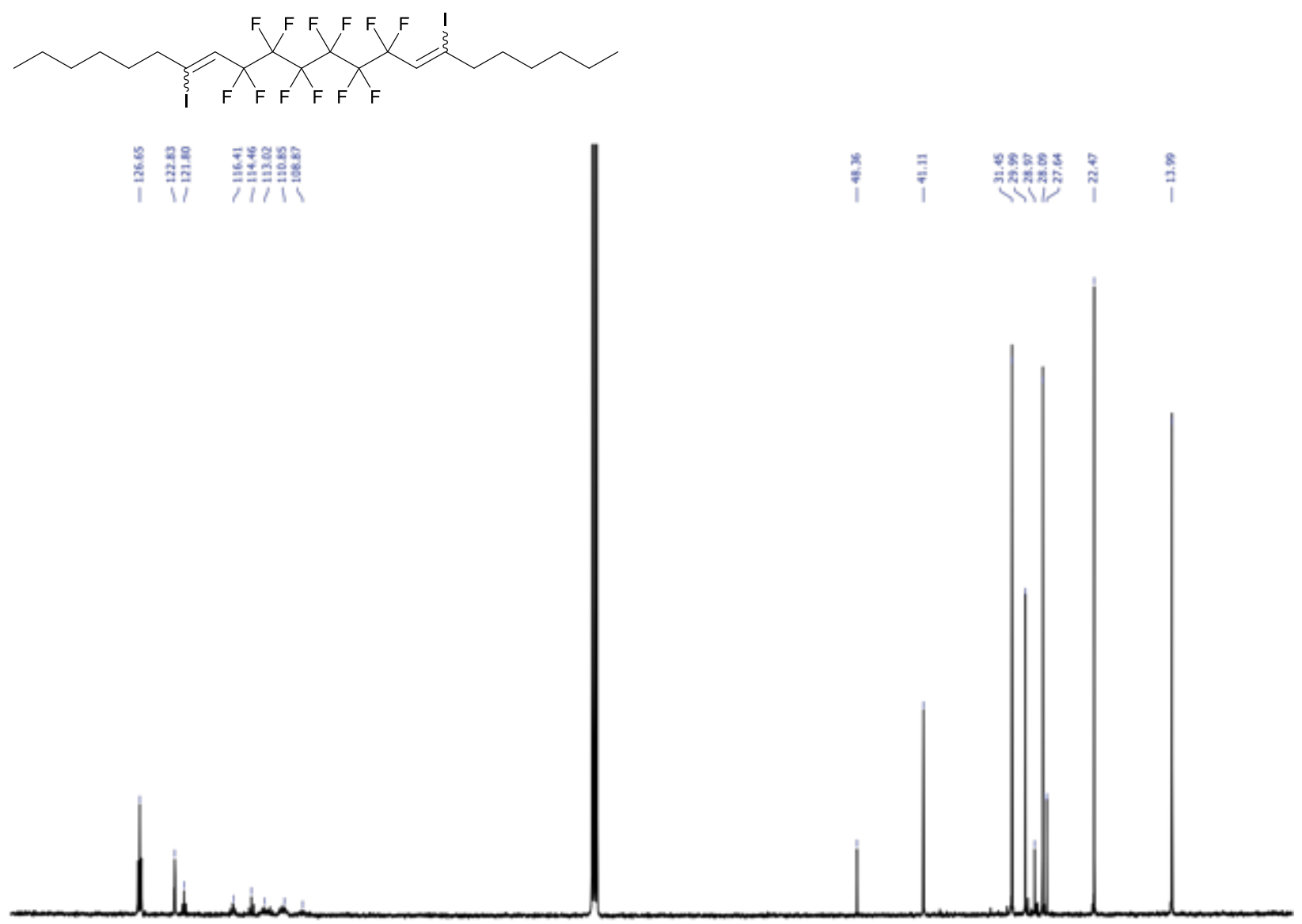

$\begin{array}{llllllllllllllllllllllllllll}140 & 135 & 130 & 125 & 120 & 115 & 110 & 105 & 100 & 95 & 90 & 85 & 80 & 75 & 70 & 65 & 60 & 55 & 50 & 45 & 40 & 35 & 30 & 25 & 20 & 15 & 10 & 5\end{array}$ 
$((9,9,10,10,11,11,12,12,13,13,14,14,14-$ tridecafluorotetradec-7-en-7-yl)oxy)benzene, S4:

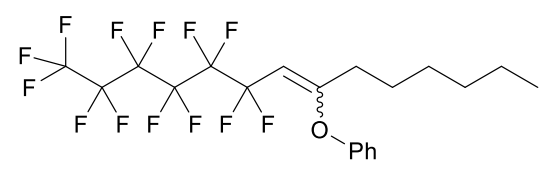
1

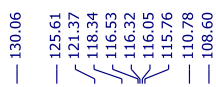

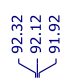

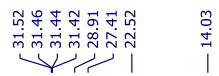

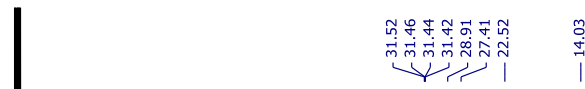

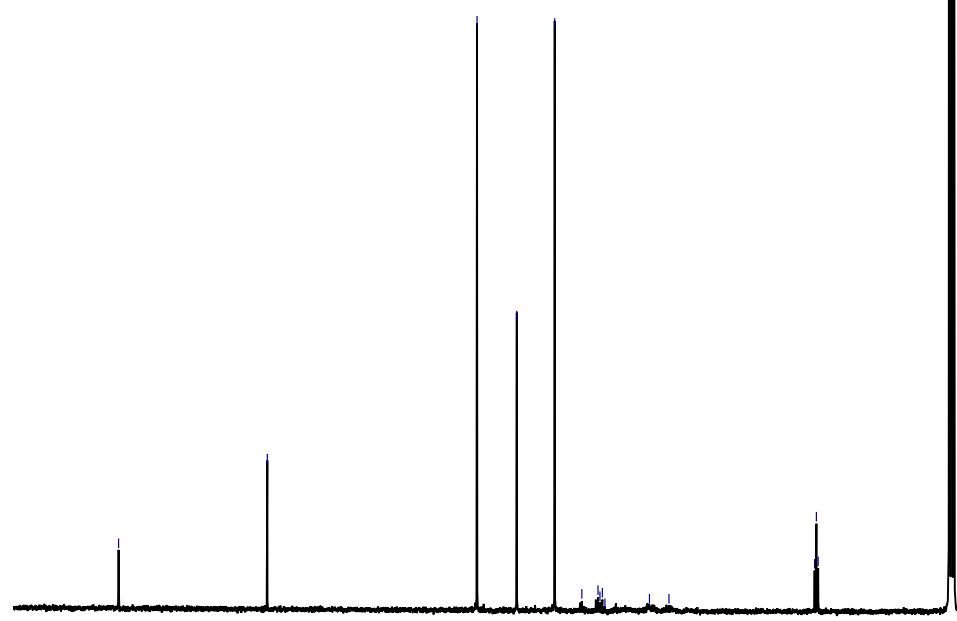

180

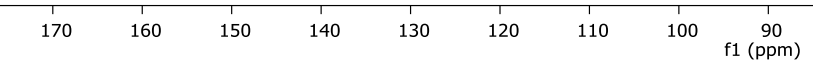

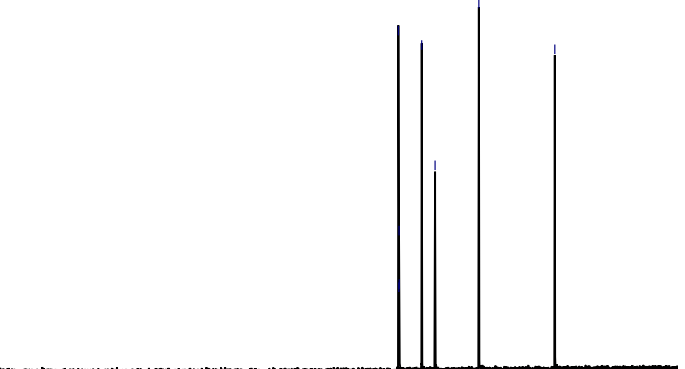


1,1,1,2,2,3,3,4,4,5,5,6-dodecafluorotetradec-6-en-8-yne, S5:
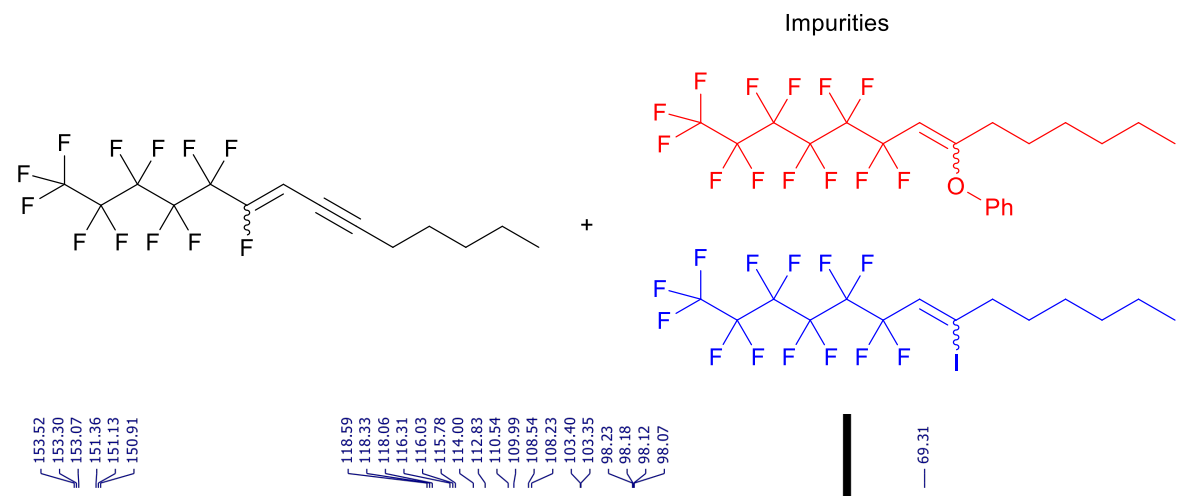

斗齊 等 m

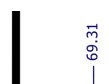

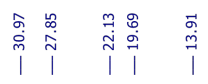

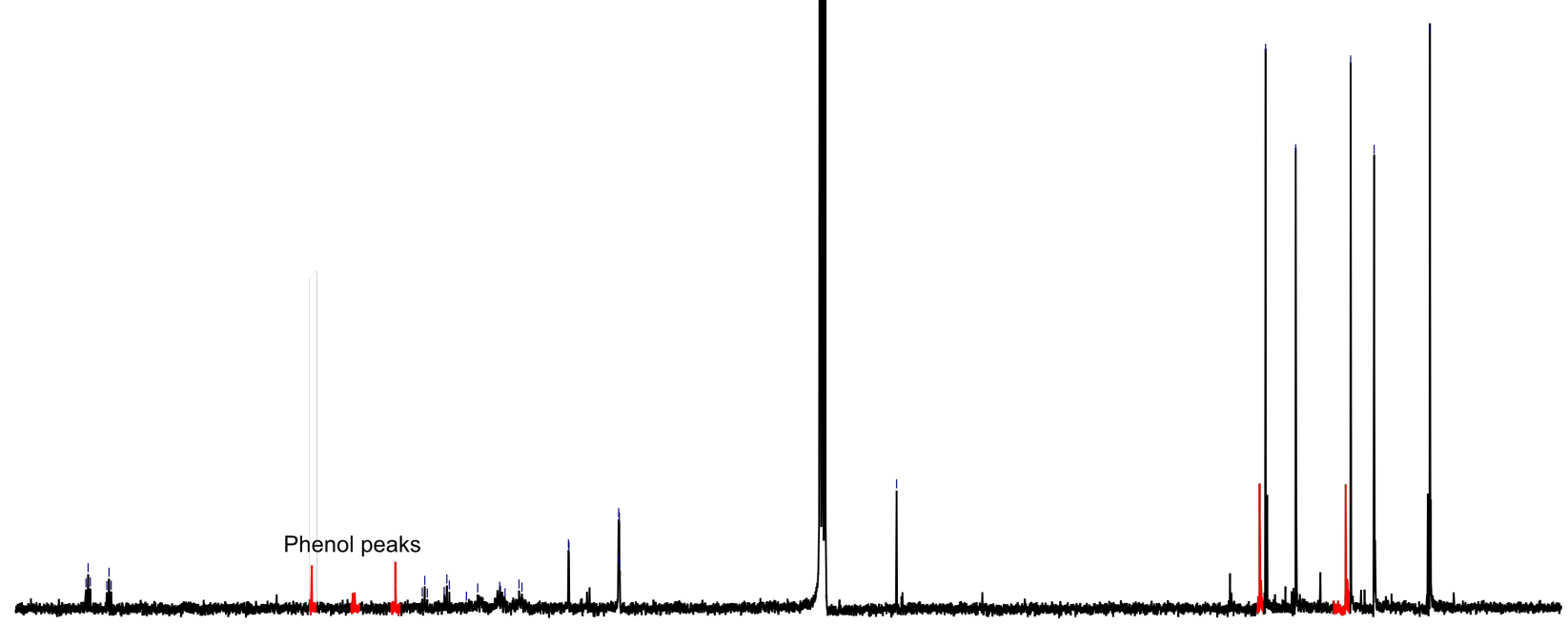

160 130 120 110 100 90 80
$1(\mathrm{ppm})$

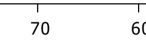

$60 \quad 50$ 


\section{${ }^{19}$ F NMR Spectra:}

9,9,10,10,11,11,12,12,13,13,14,14-dodecafluoro-7,16-diiododocosa-7,15-diene, S1, S2:
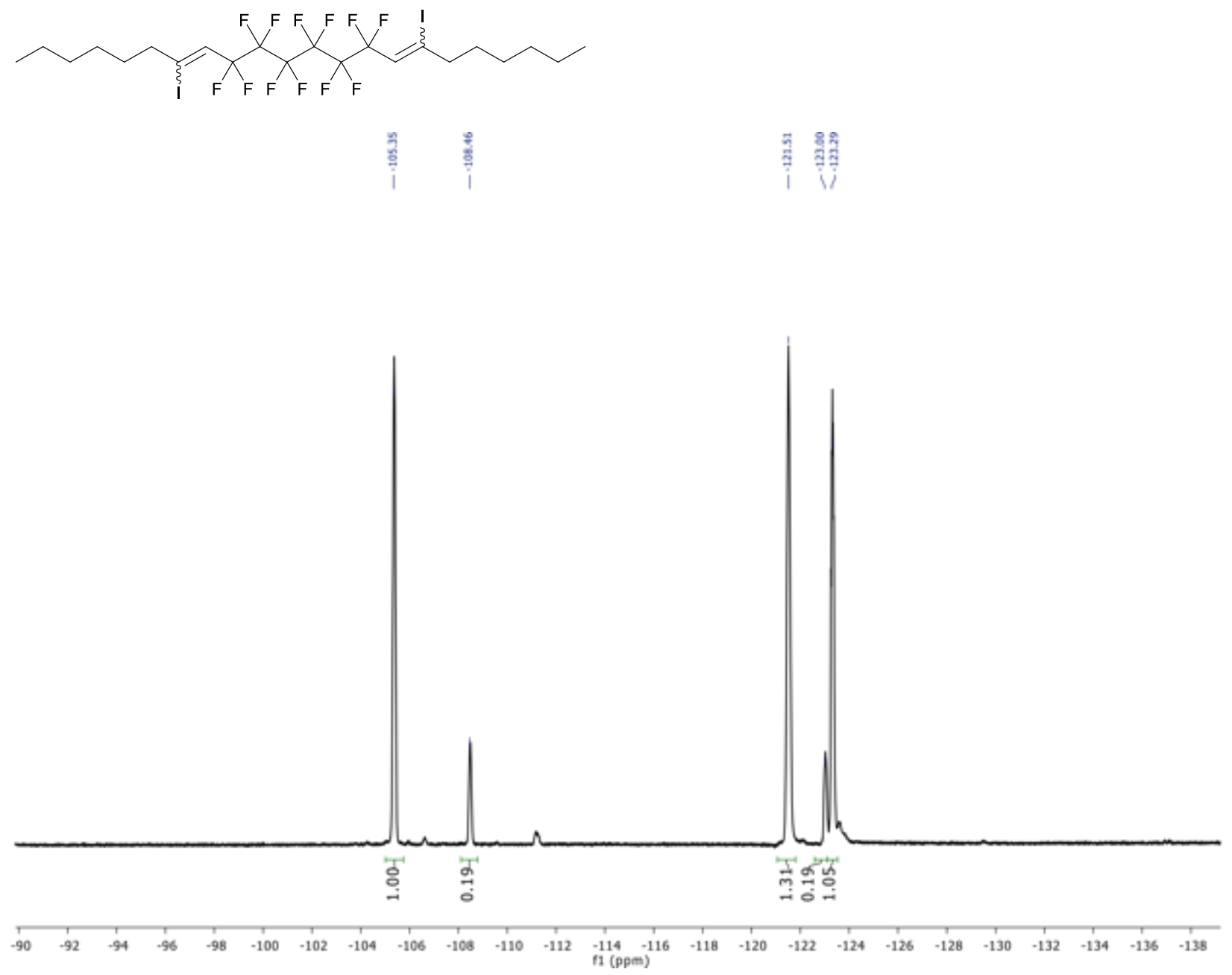
$((9,9,10,10,11,11,12,12,13,13,14,14,14-$ tridecafluorotetradec-7-en-7-yl)oxy)benzene, S4:
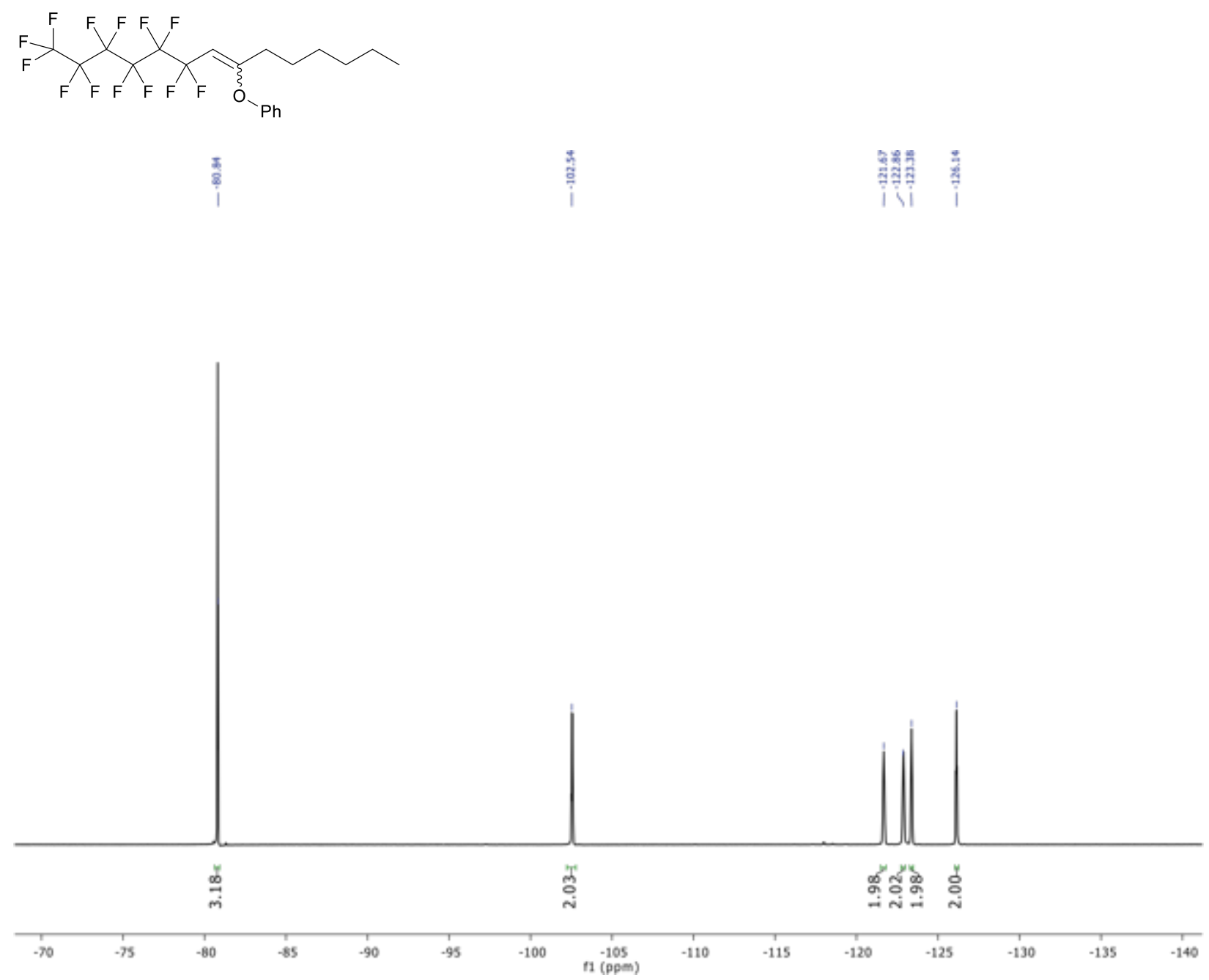


\section{1,1,1,2,2,3,3,4,4,5,5,6-dodecafluorotetradec-6-en-8-yne, S5:}
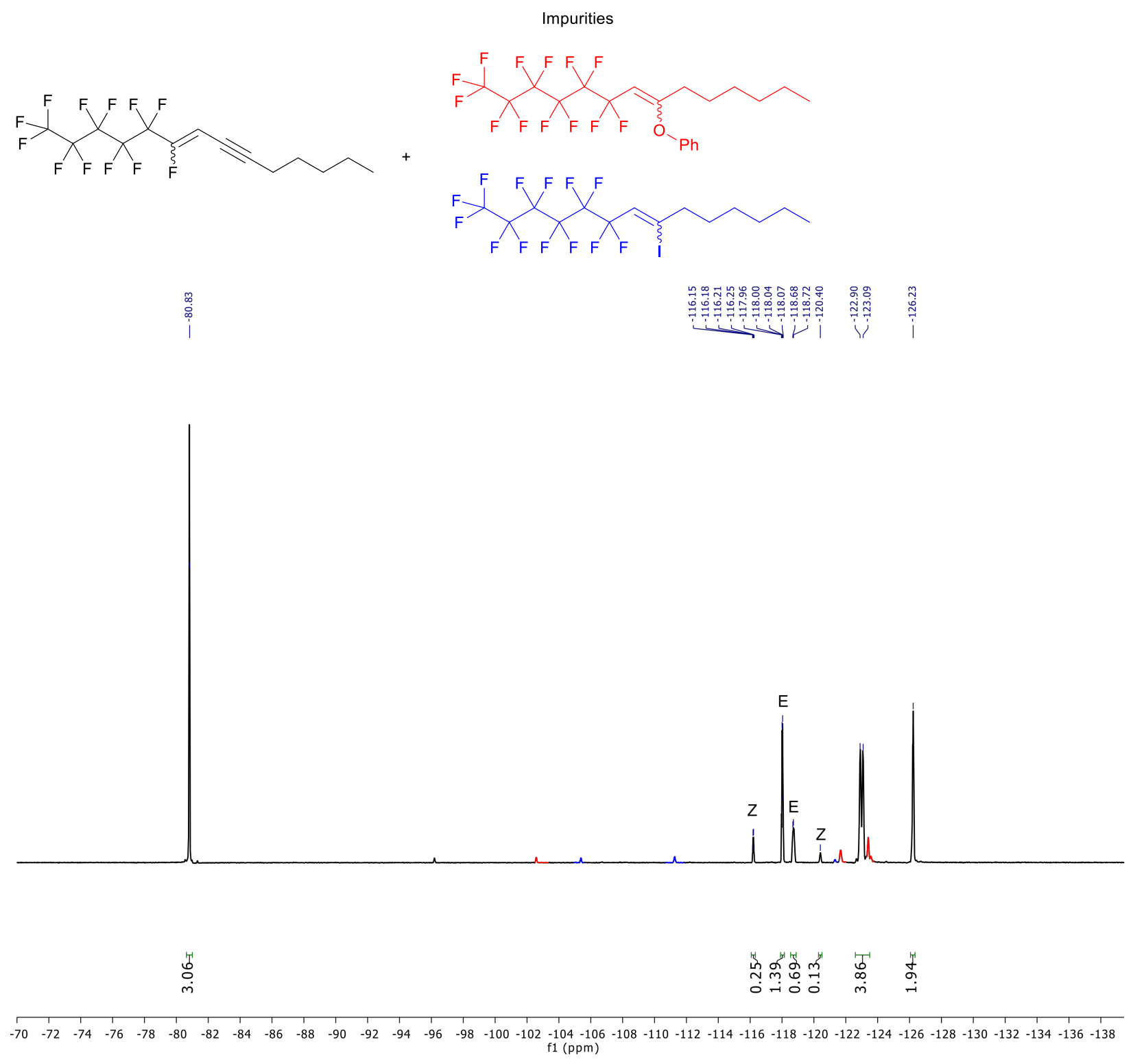
1-perfluorohexyl-2,9-diiodo-1,9-decadiene block polymer, 3:
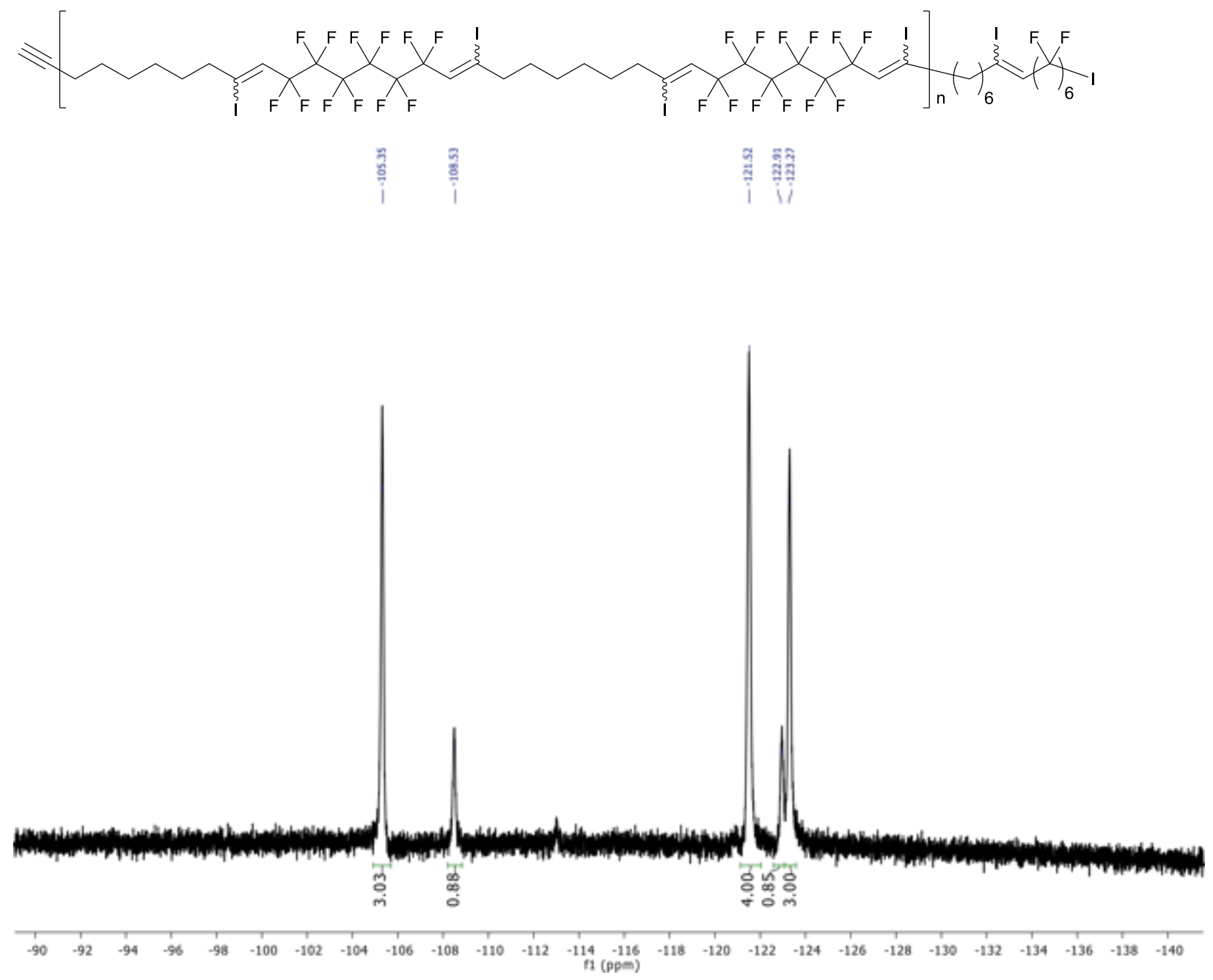
Thiol capping of polymer 3,4 :
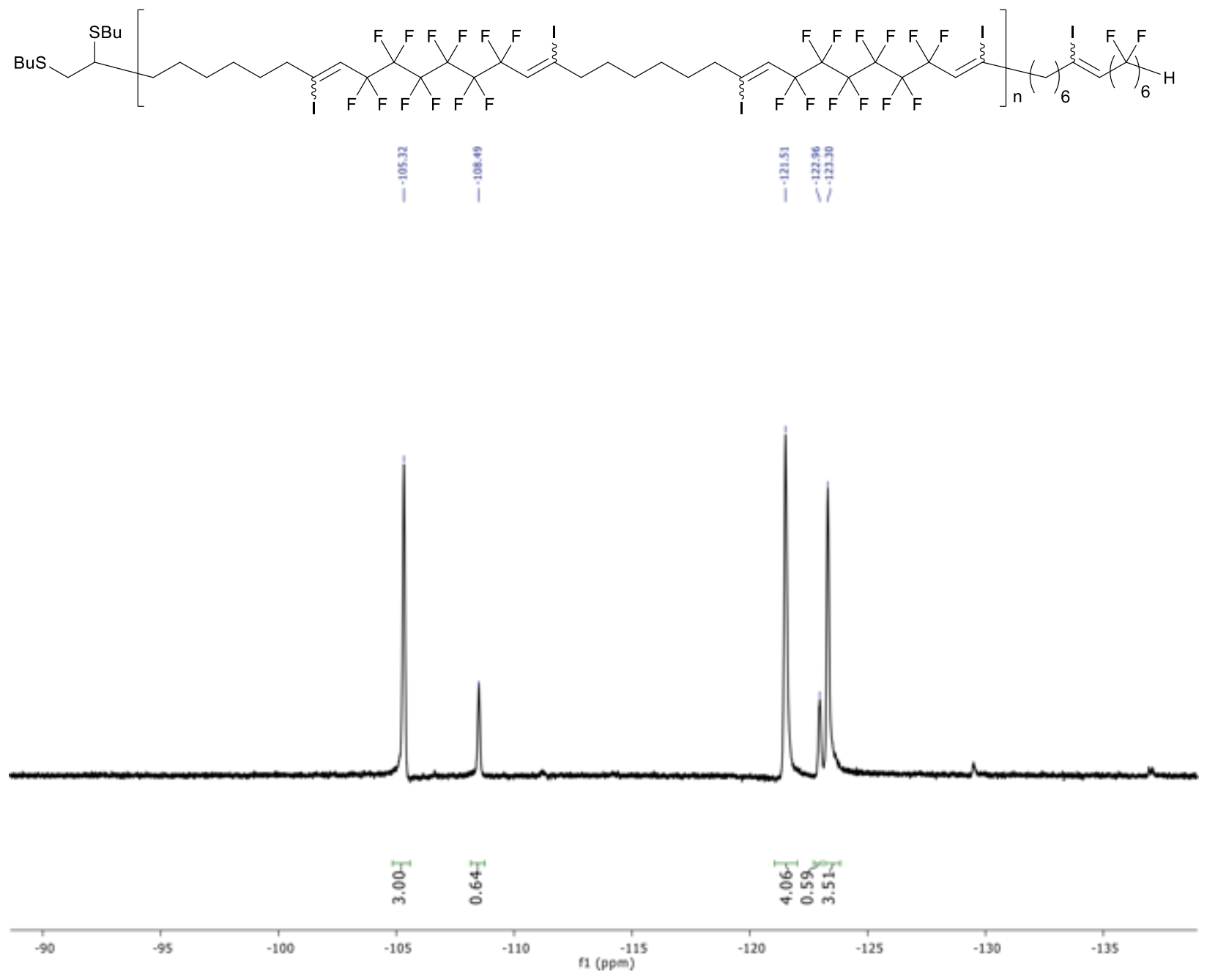
Sonogashira coupling of polymer 4,5 :
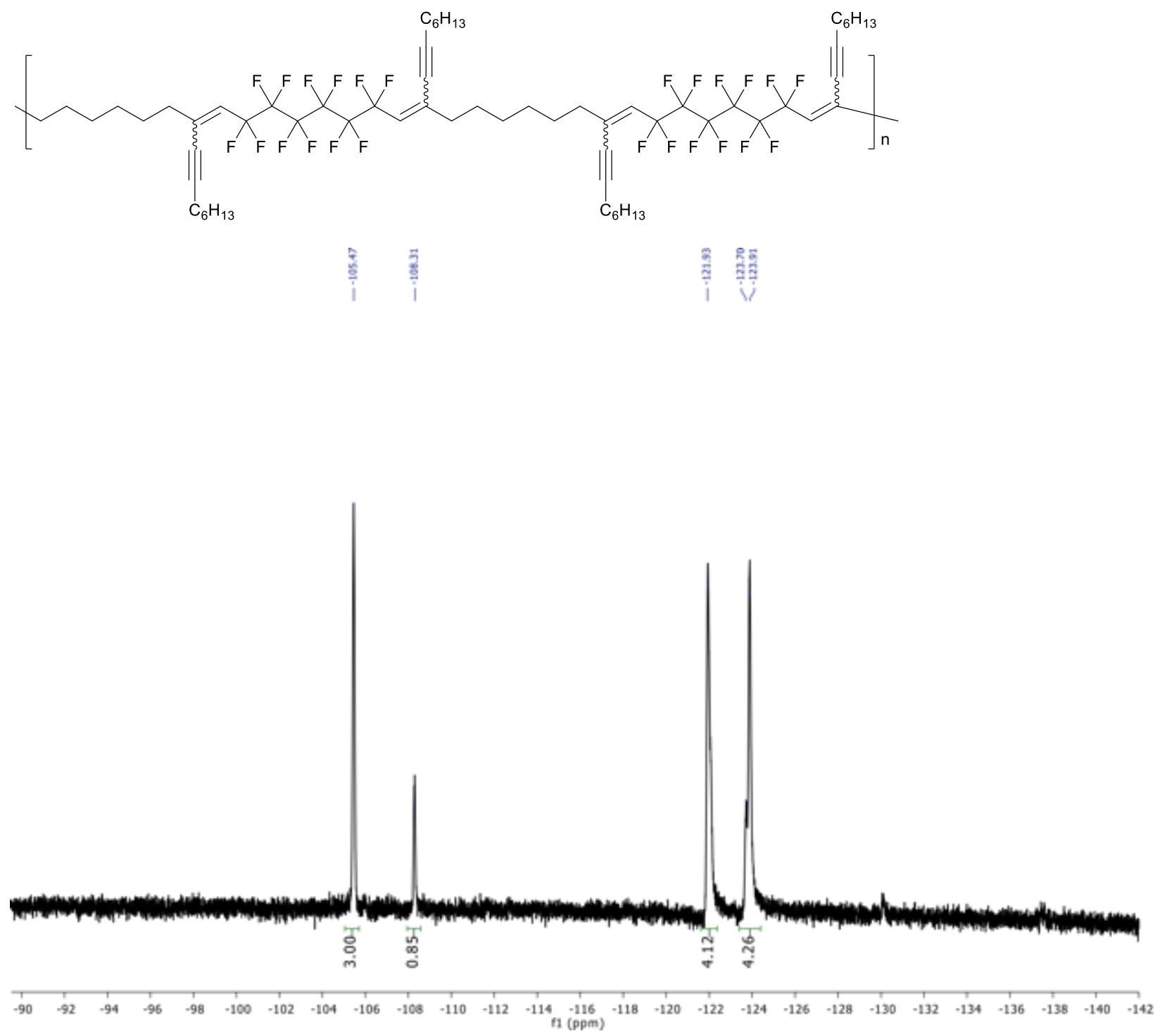
Stille coupling of polymer 4,6 :
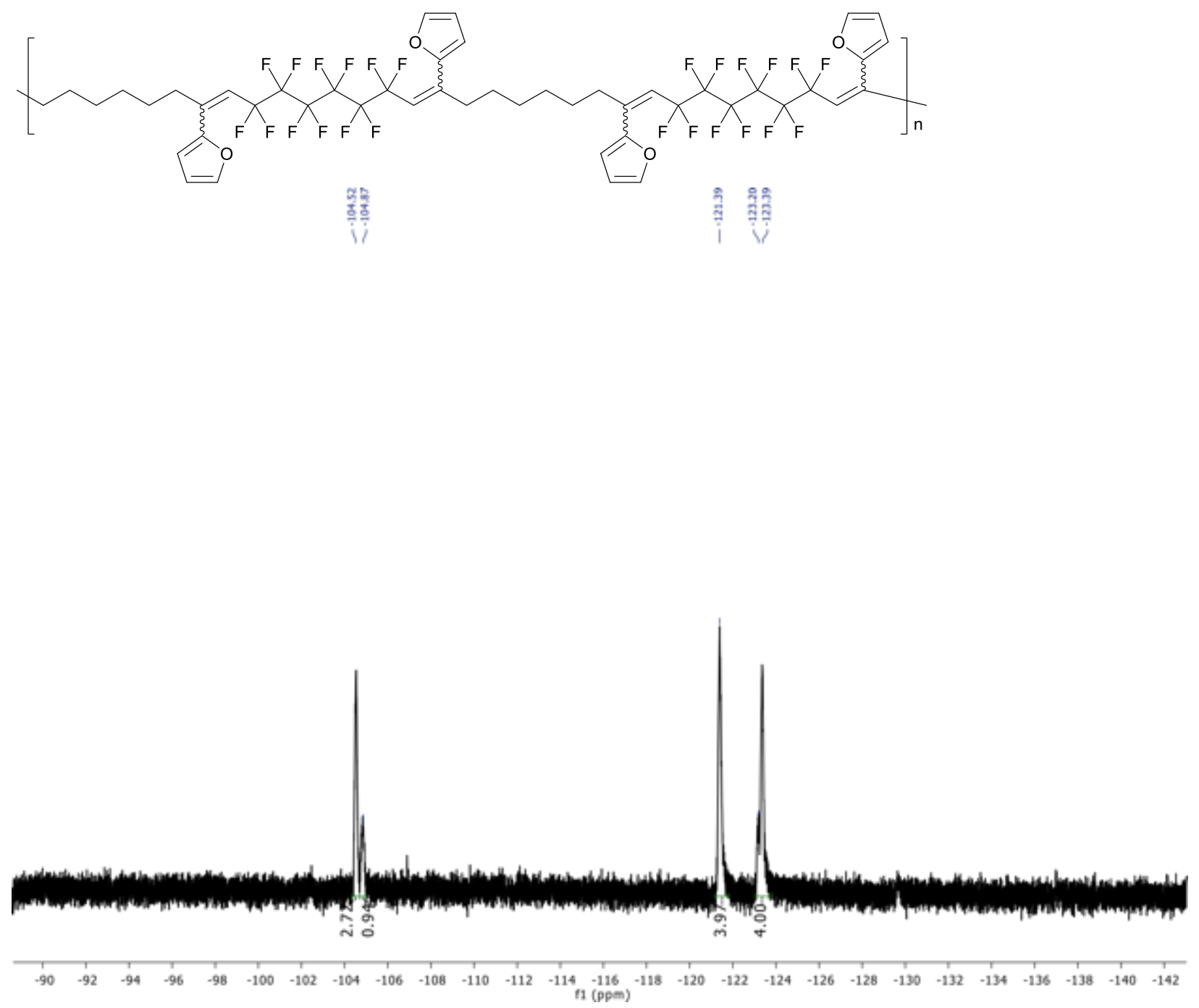
Suzuki coupling of polymer 4, 7:
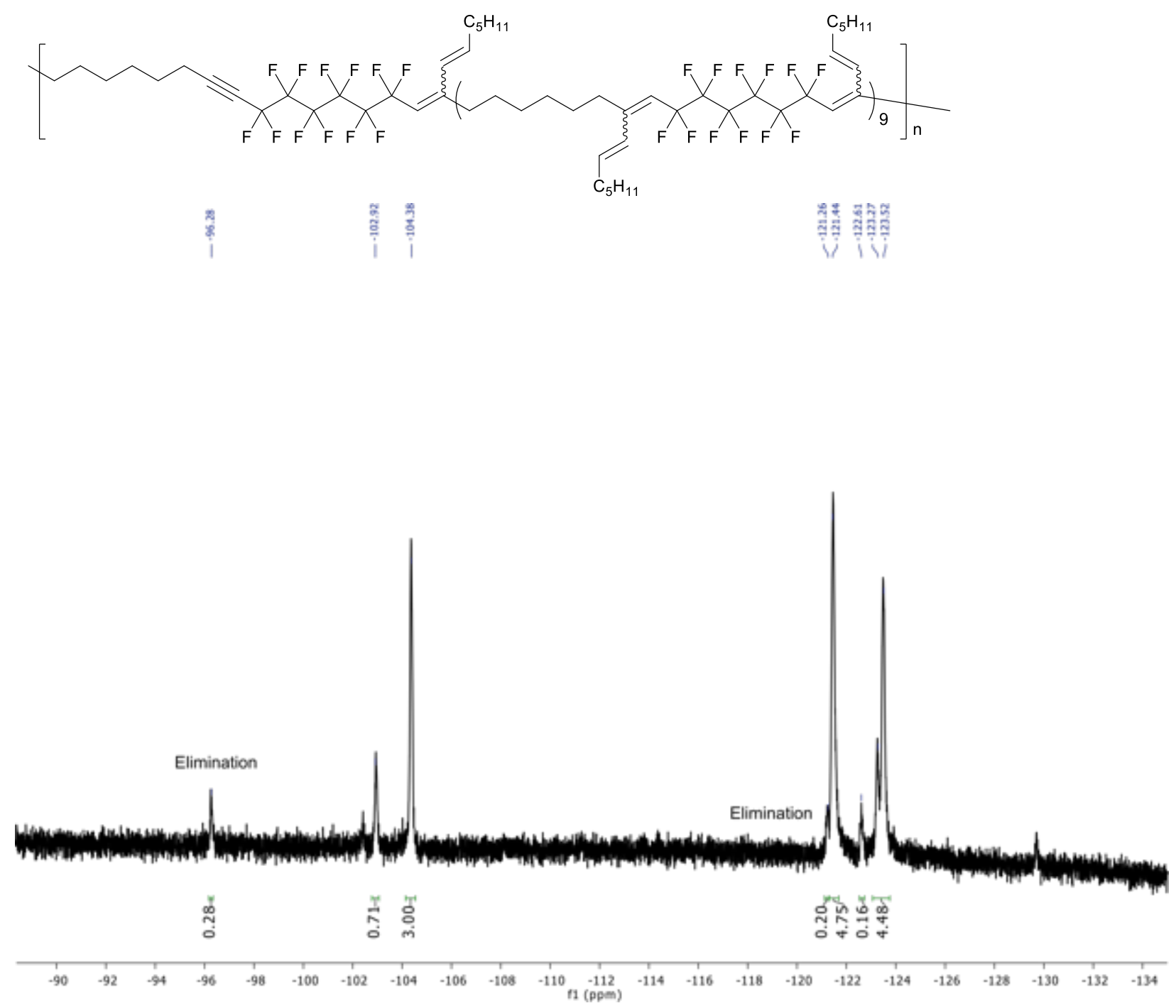
Phenol coupling of polymer 4, 8:
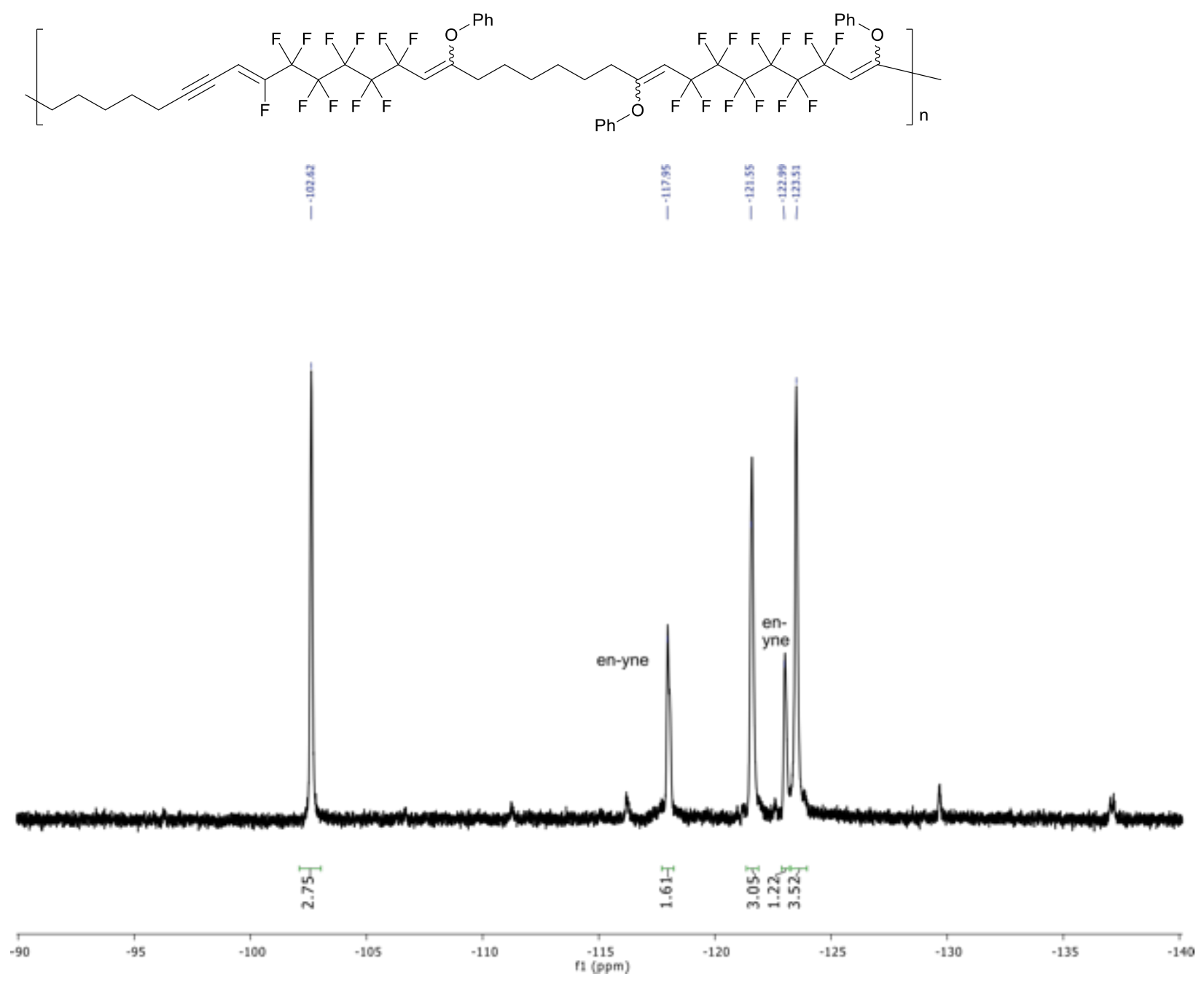
Thiophenol coupling of polymer 4, 9:
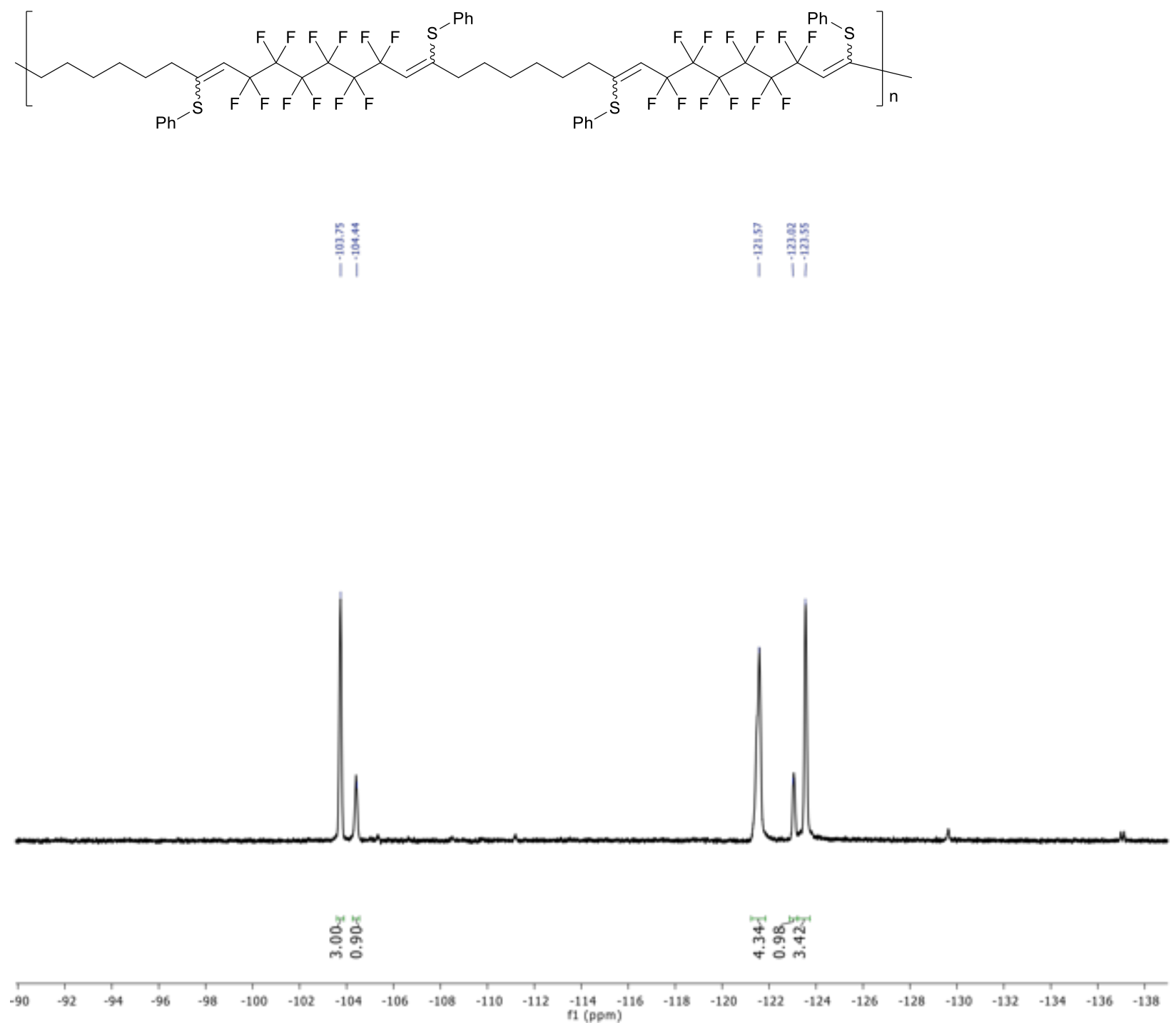
Kumada coupling of polymer 3,10 :
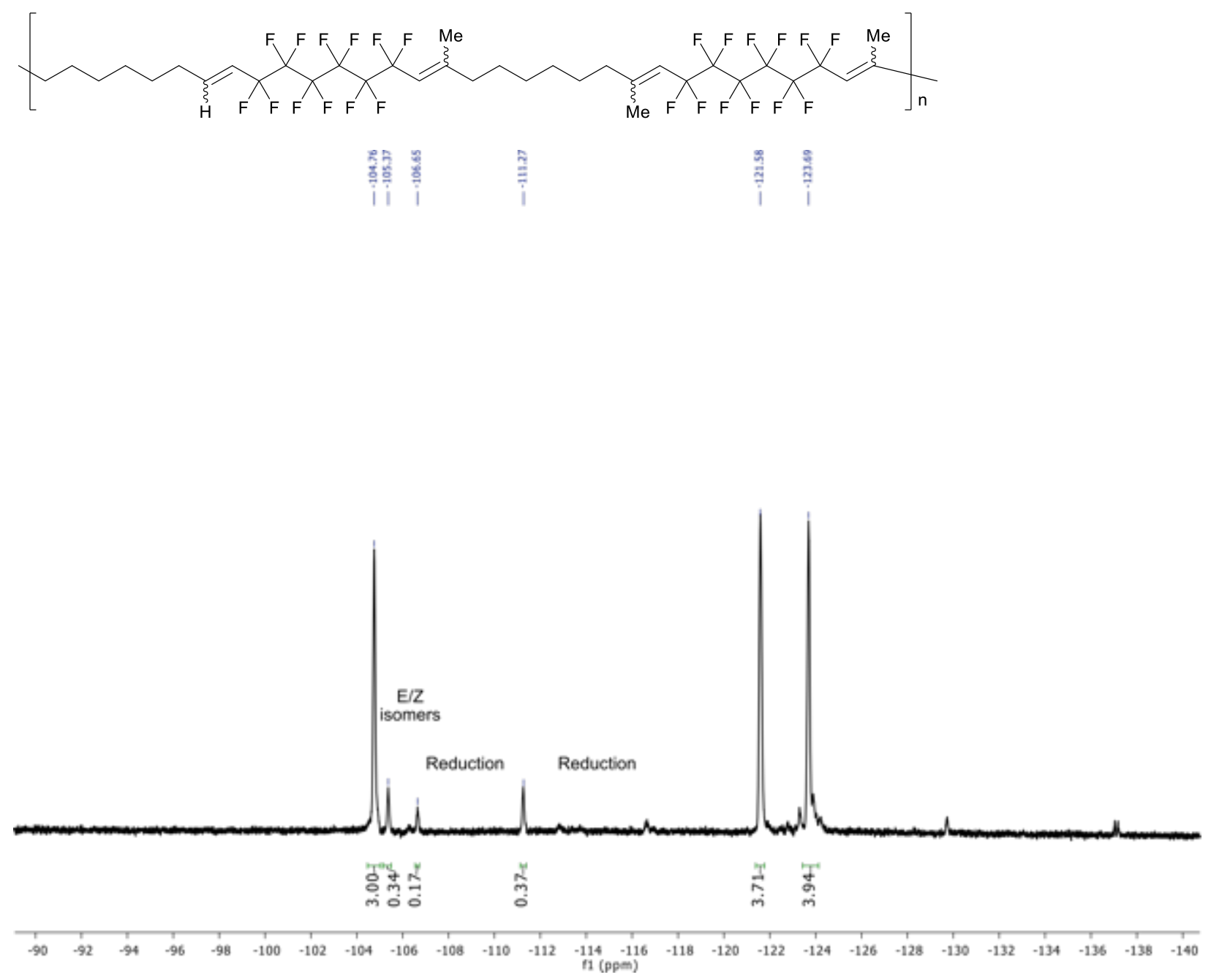
Elimination of iodide from polymer 3, 11:
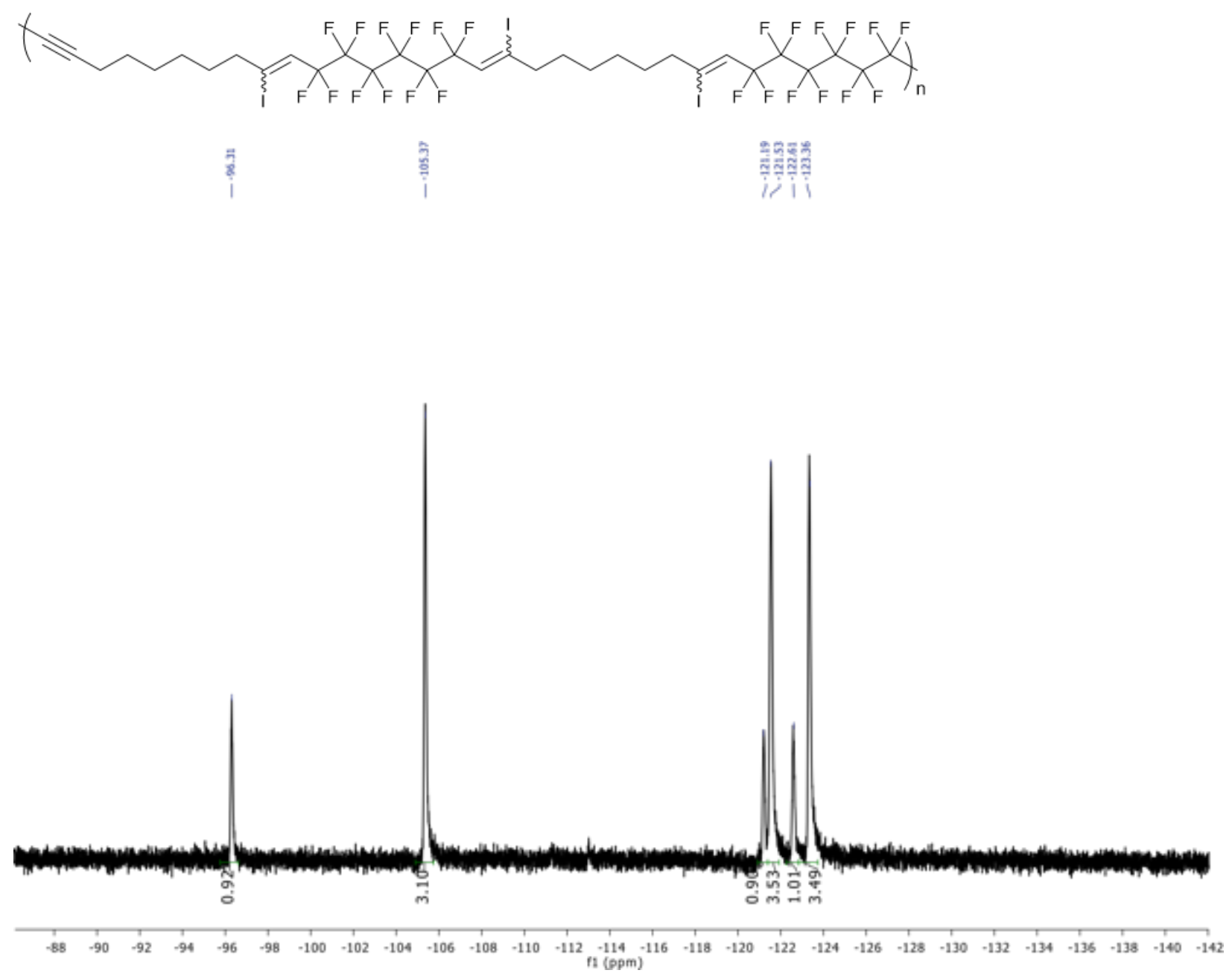
Alkyne-azide coupling of polymer 11, 12:
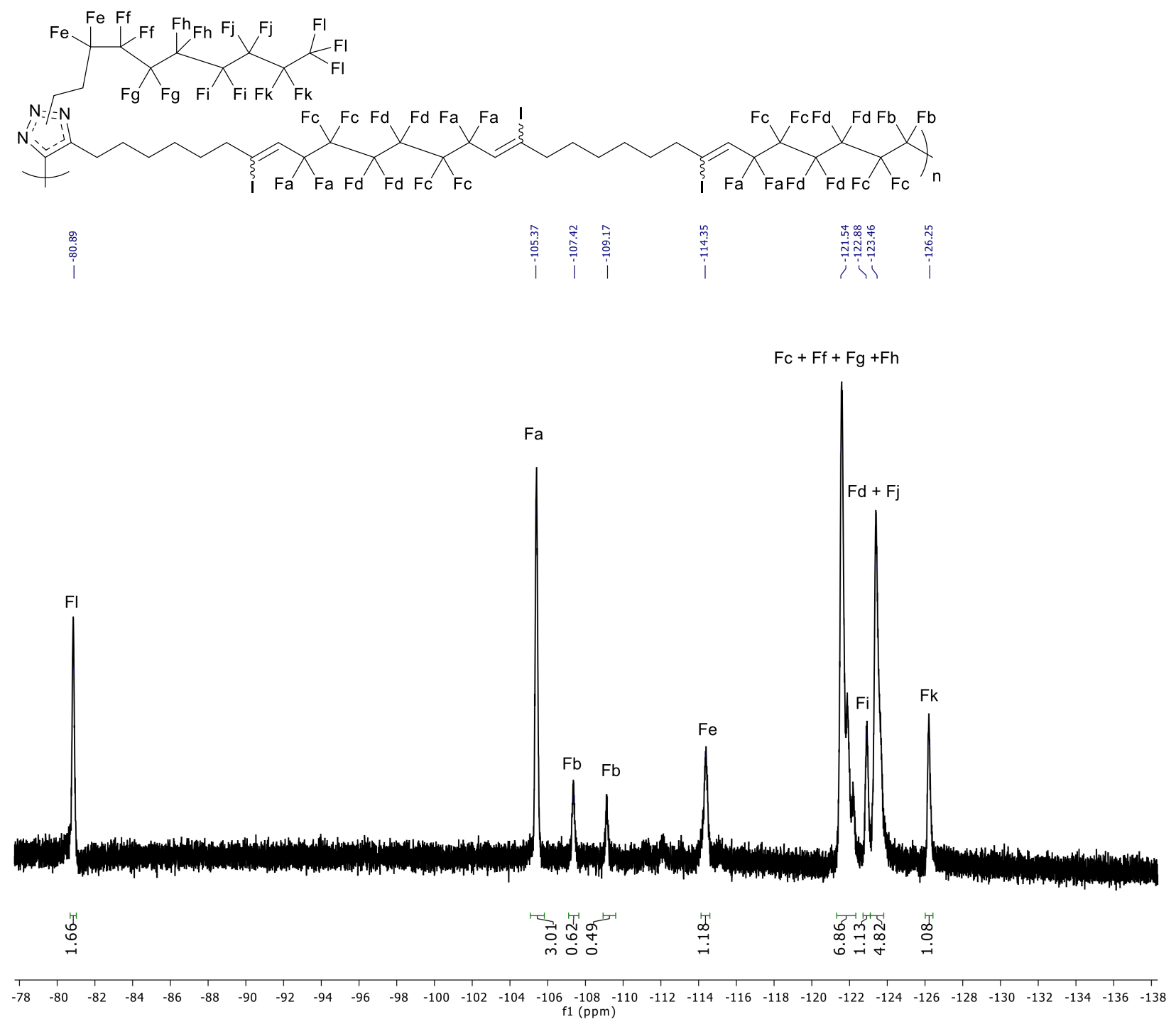


\section{IR Traces:}

1-perfluorohexyl-2,9-diiodo-1,9-decadiene block polymer, 3:
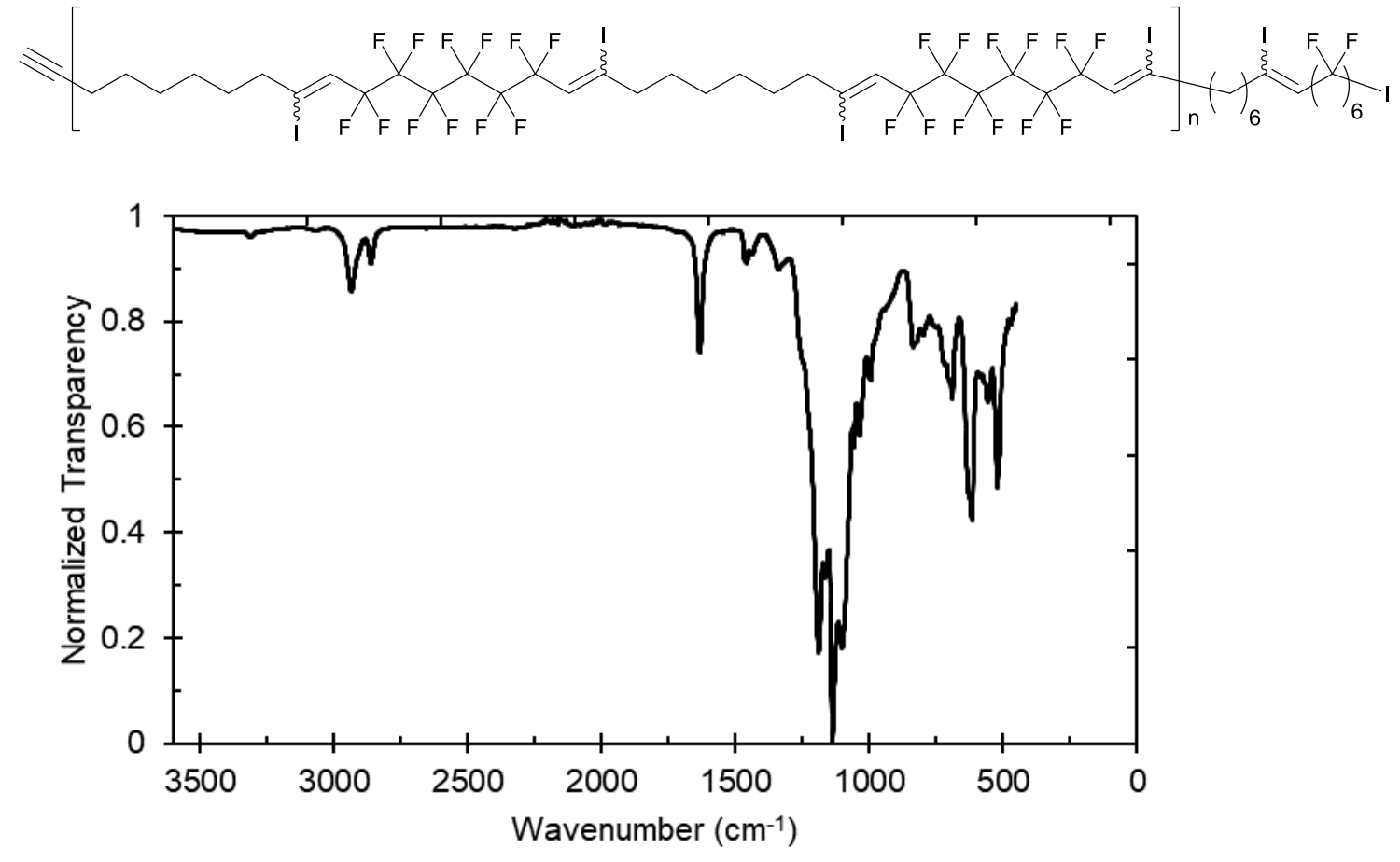
Thiol capping of polymer 3,4 :
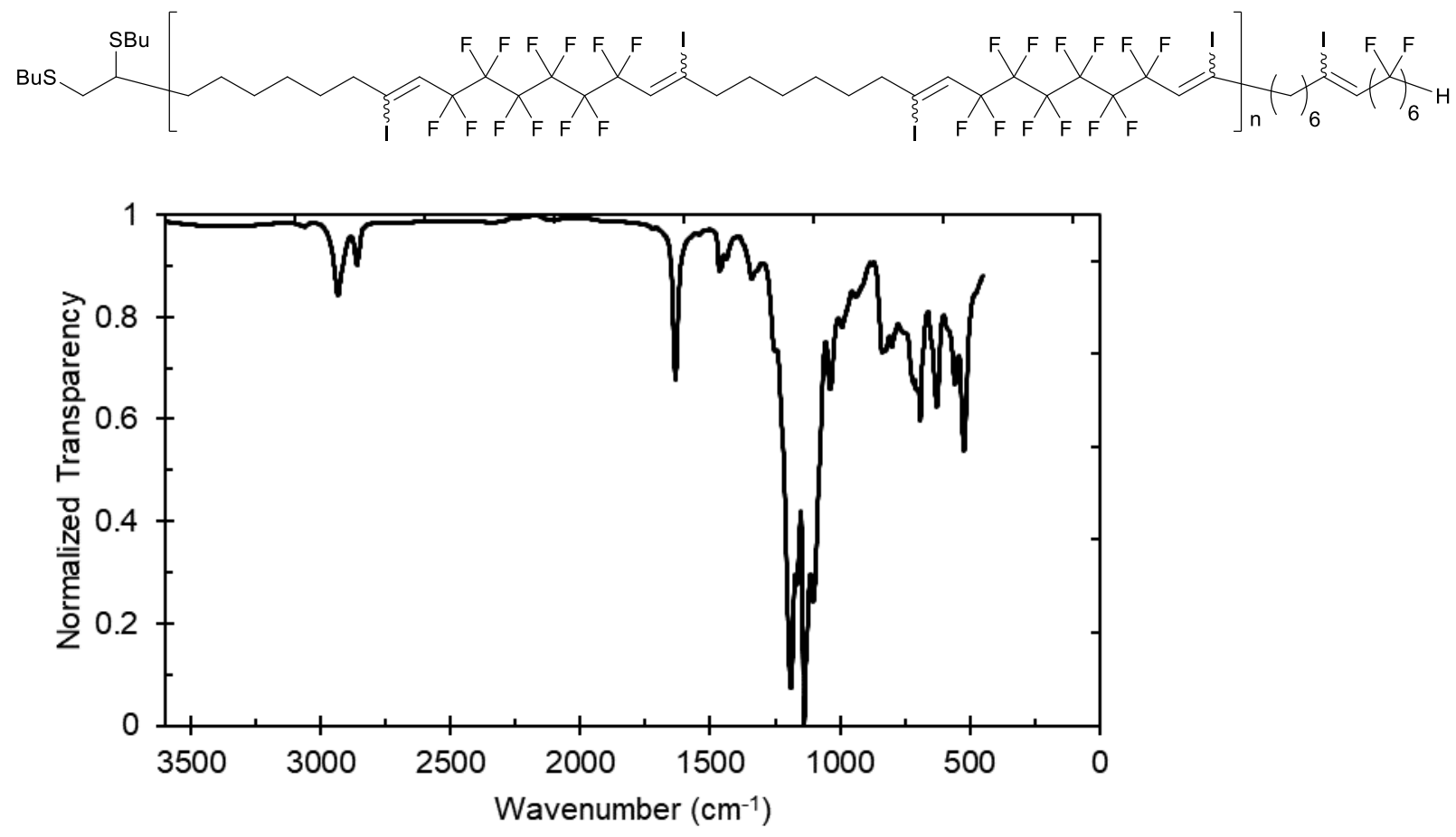
Sonogashira coupling of polymer 4, 5 :
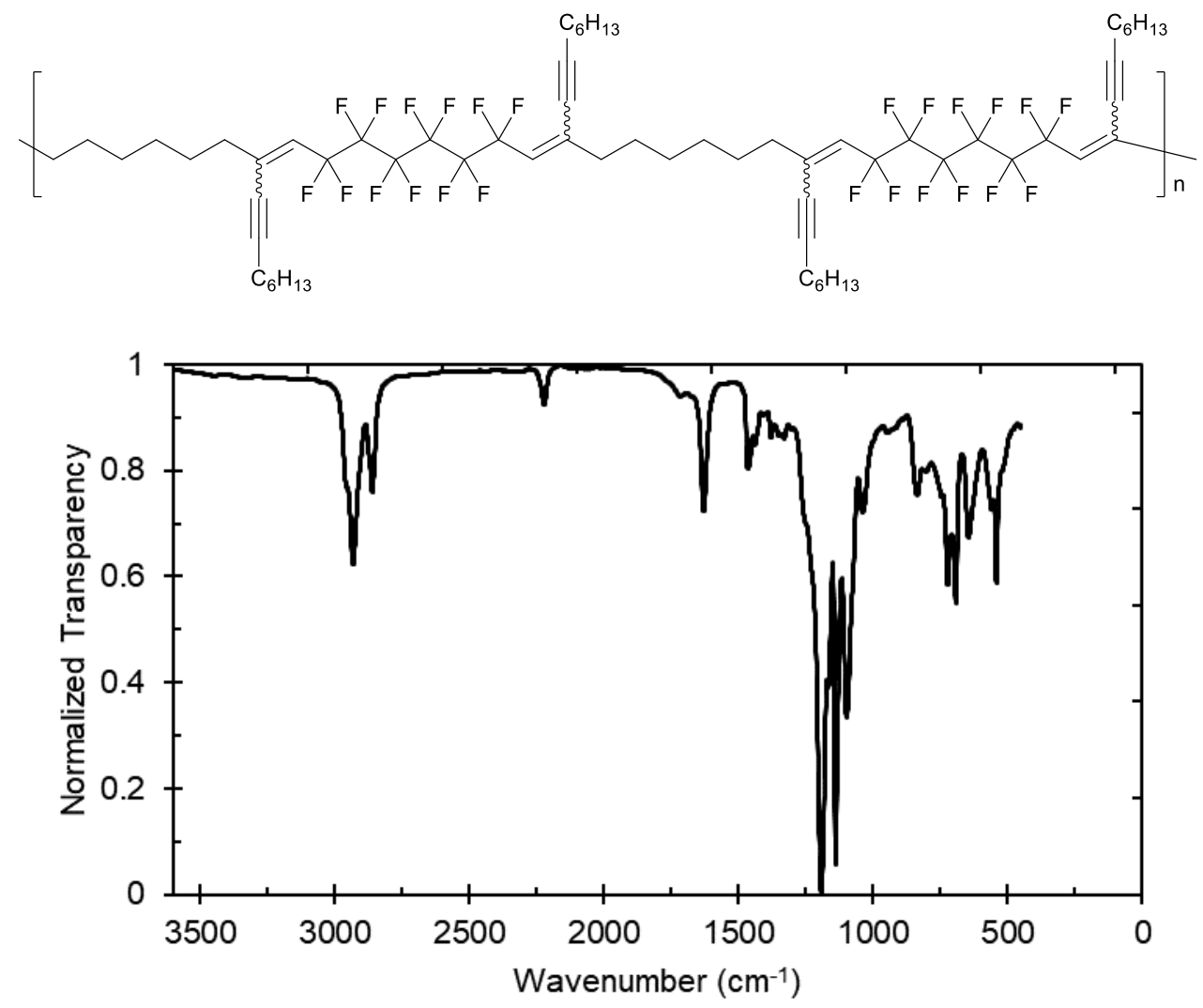
Stille coupling of polymer 4,6 :
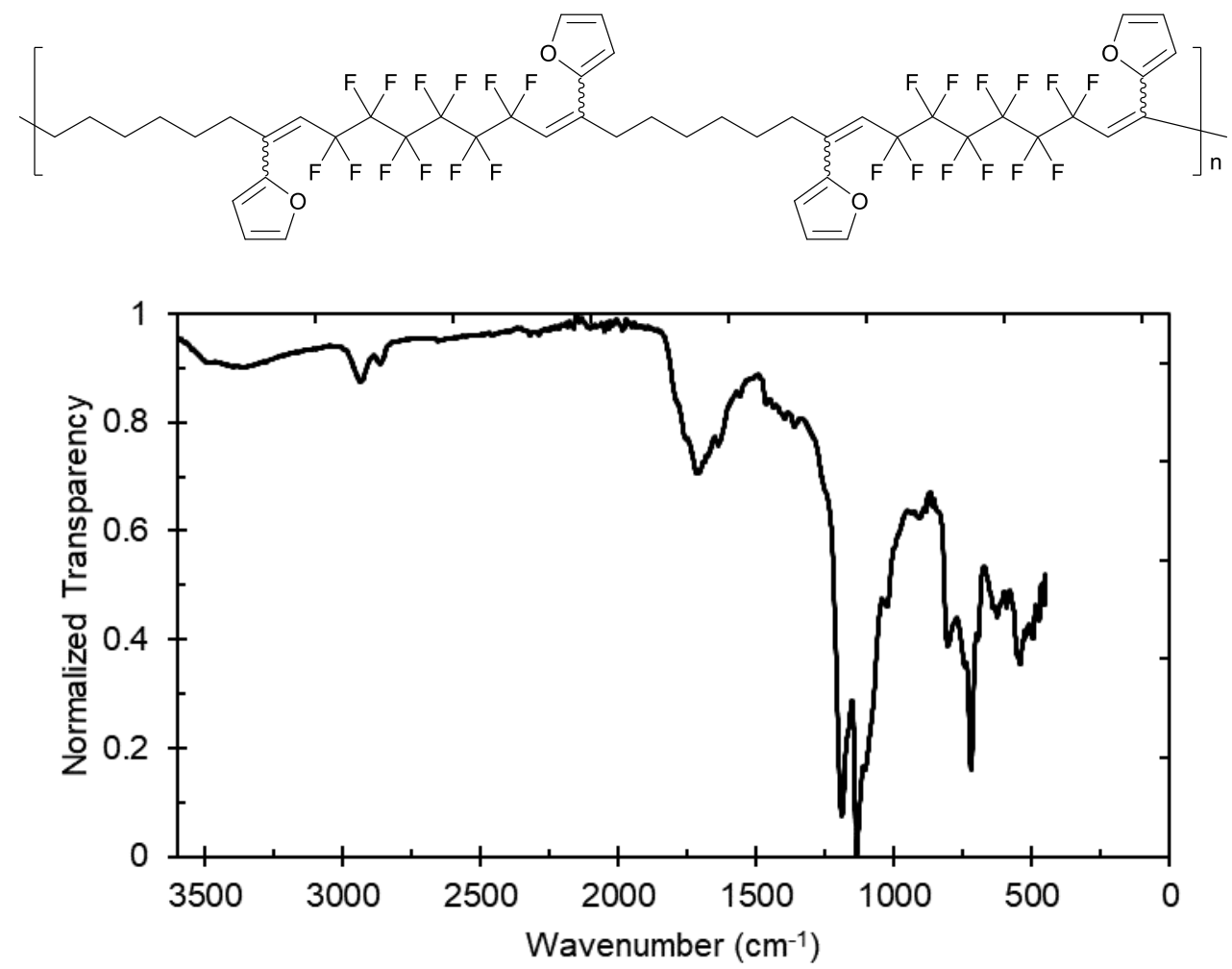
Suzuki coupling of polymer 4,7 :
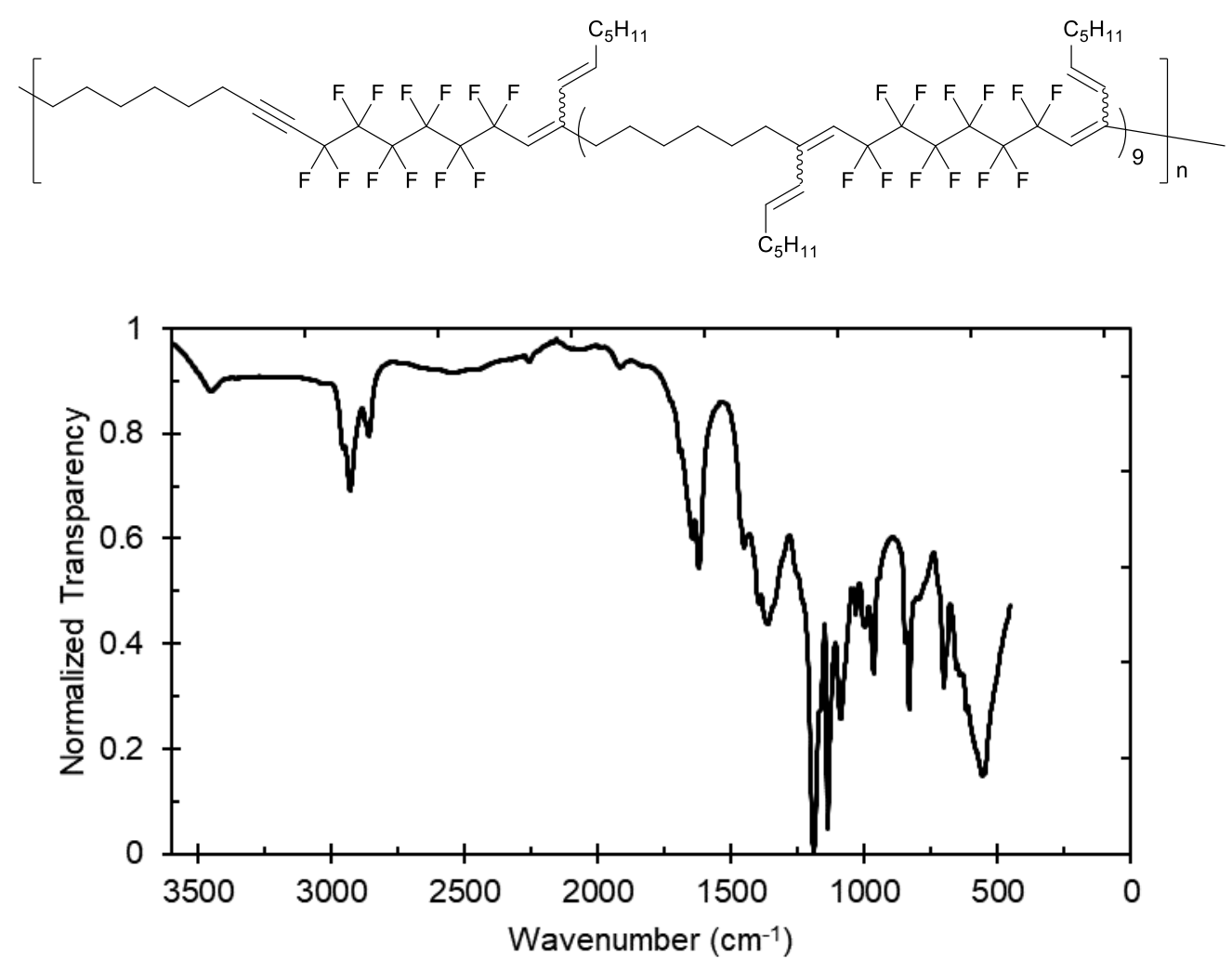
Phenol coupling of polymer 4, 8 :
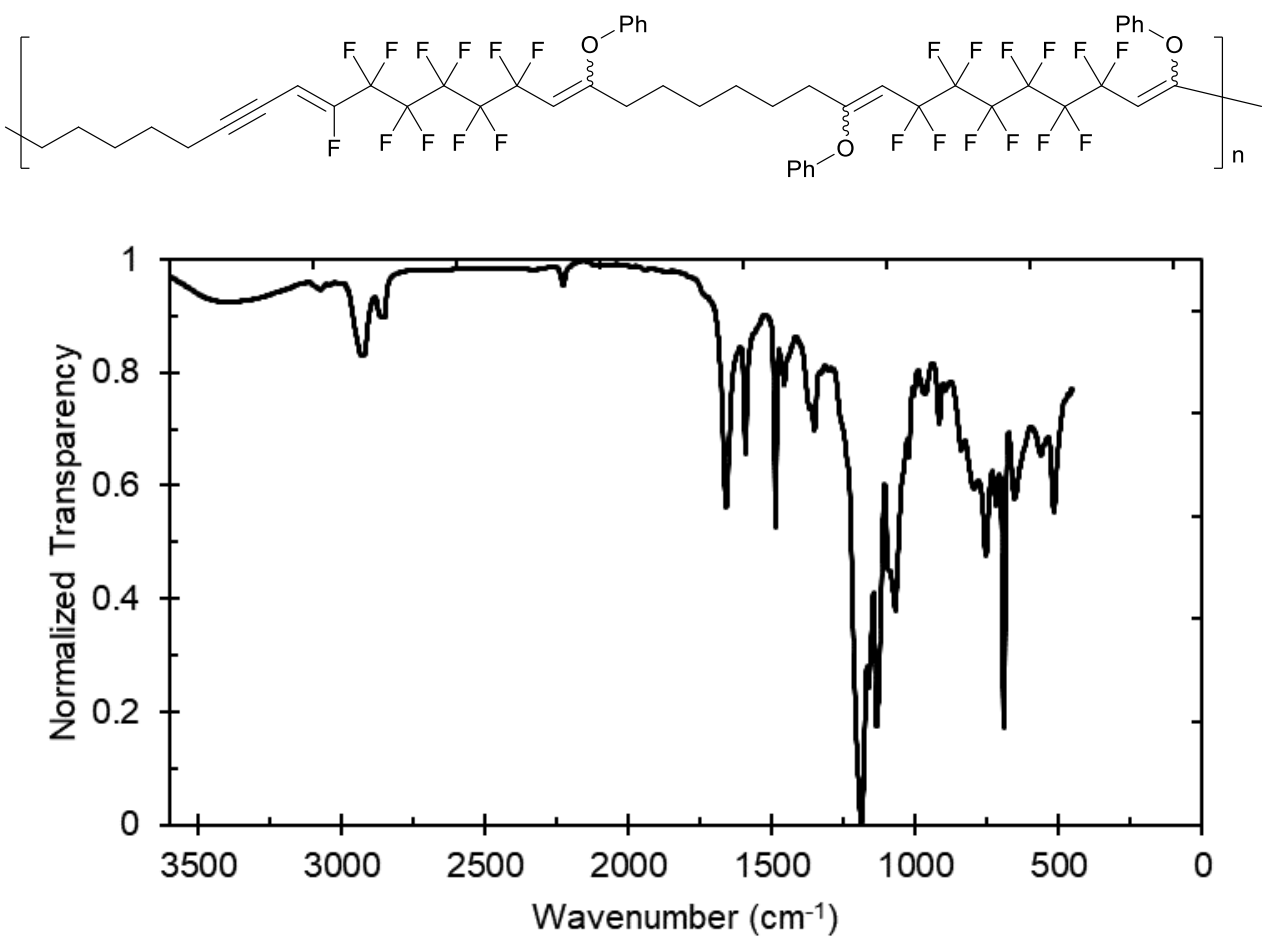
Thiophenol coupling of polymer 4, 9:
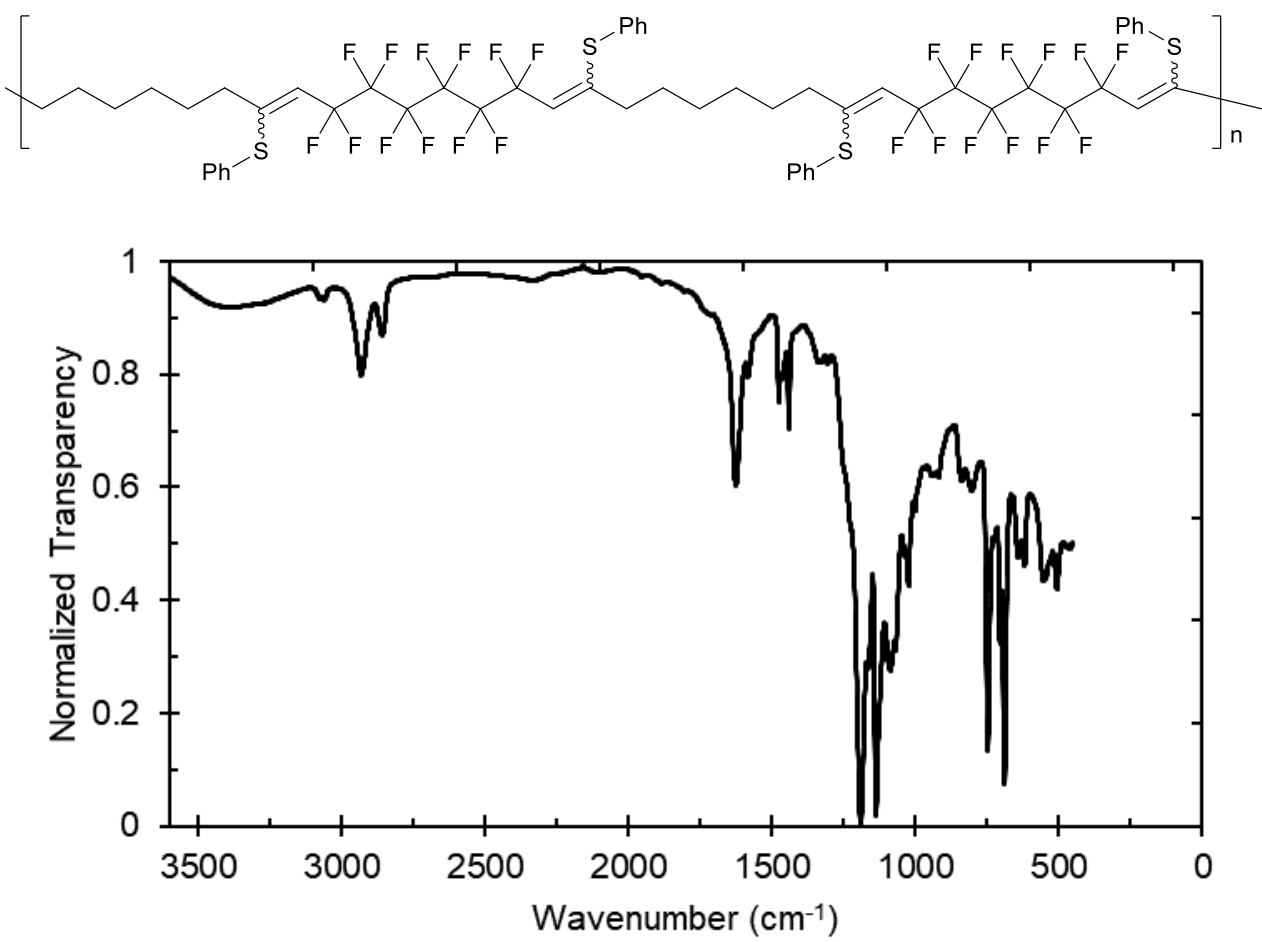
Kumada coupling of polymer 3,10 :
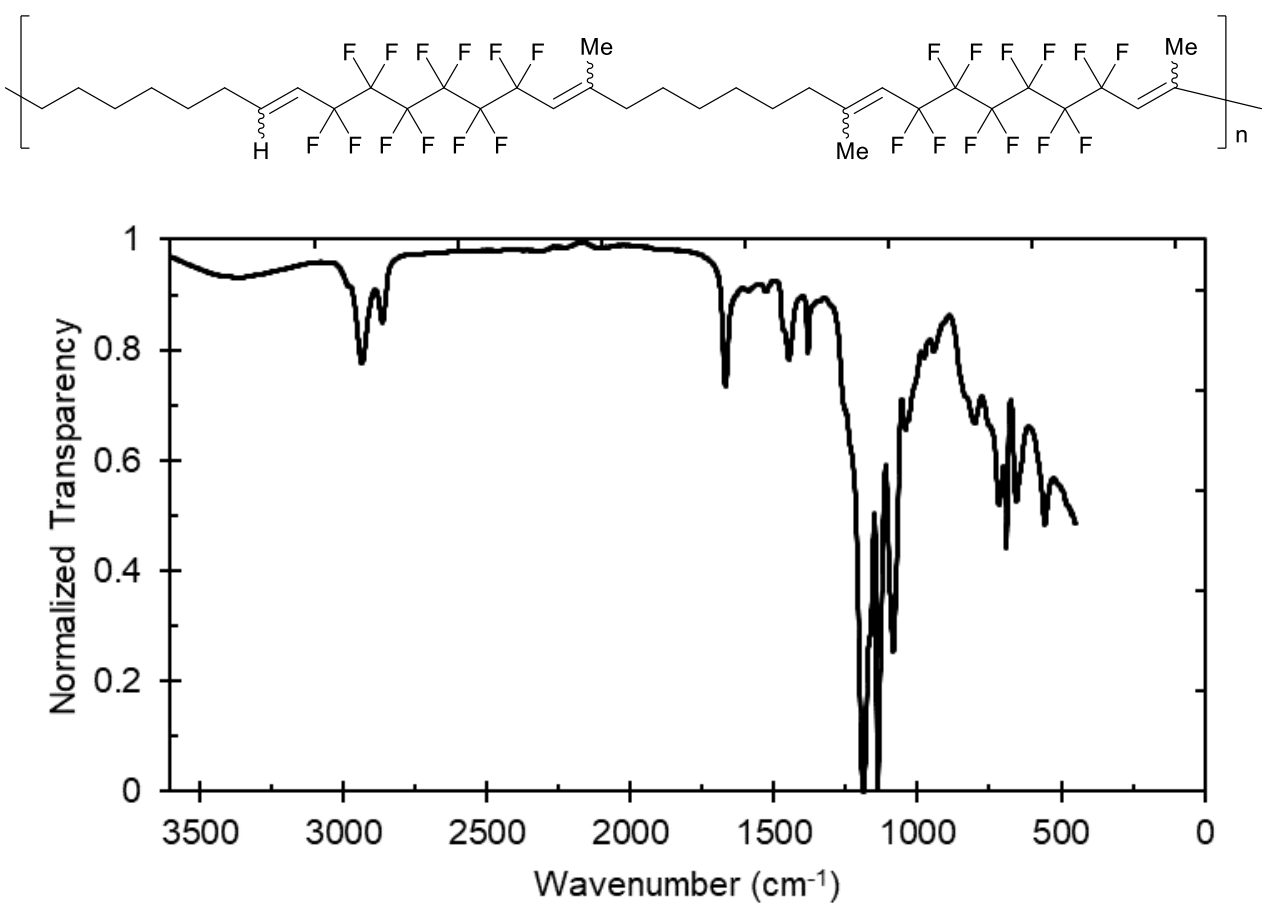
Elimination of iodide from polymer 3, 11:
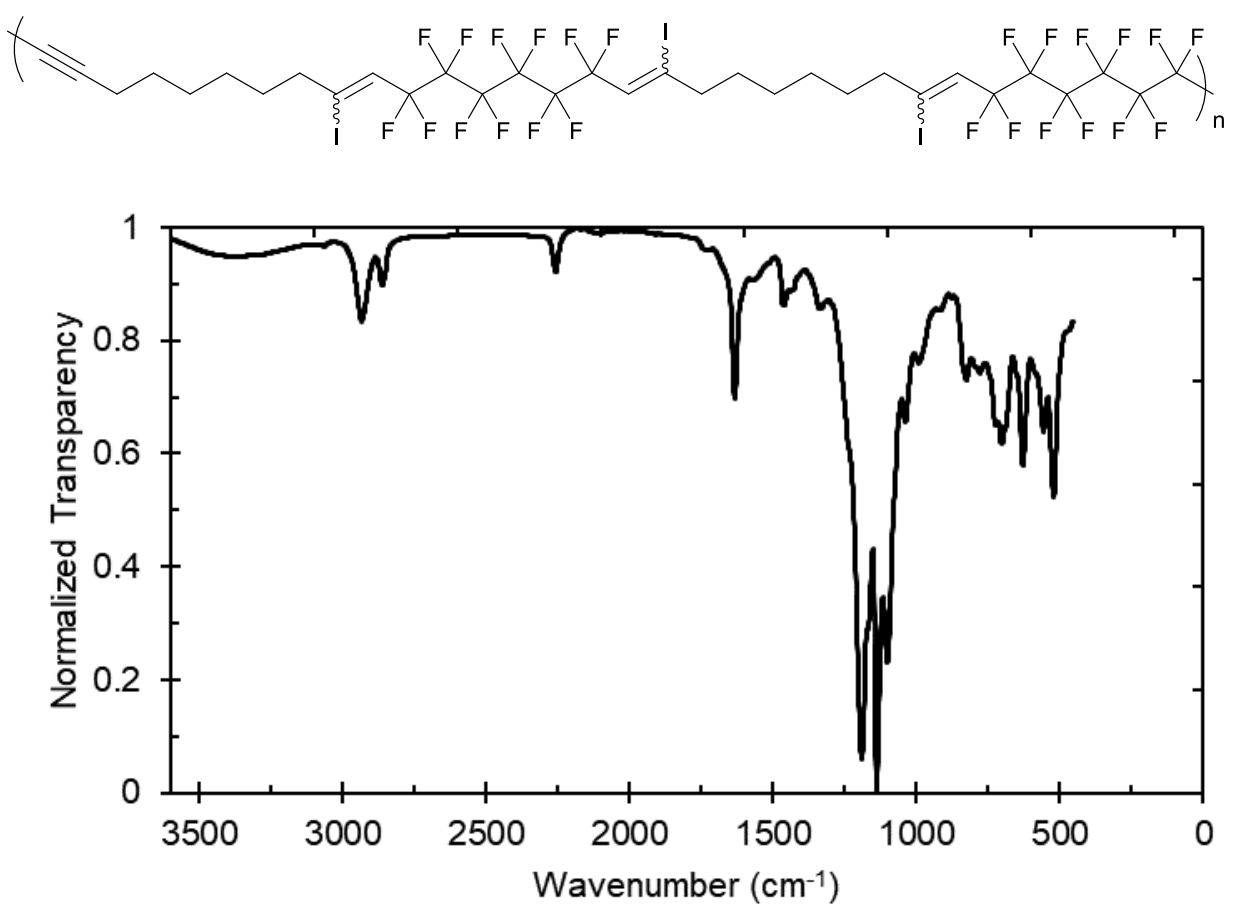
Alkyne-azide coupling of polymer 11, 12:
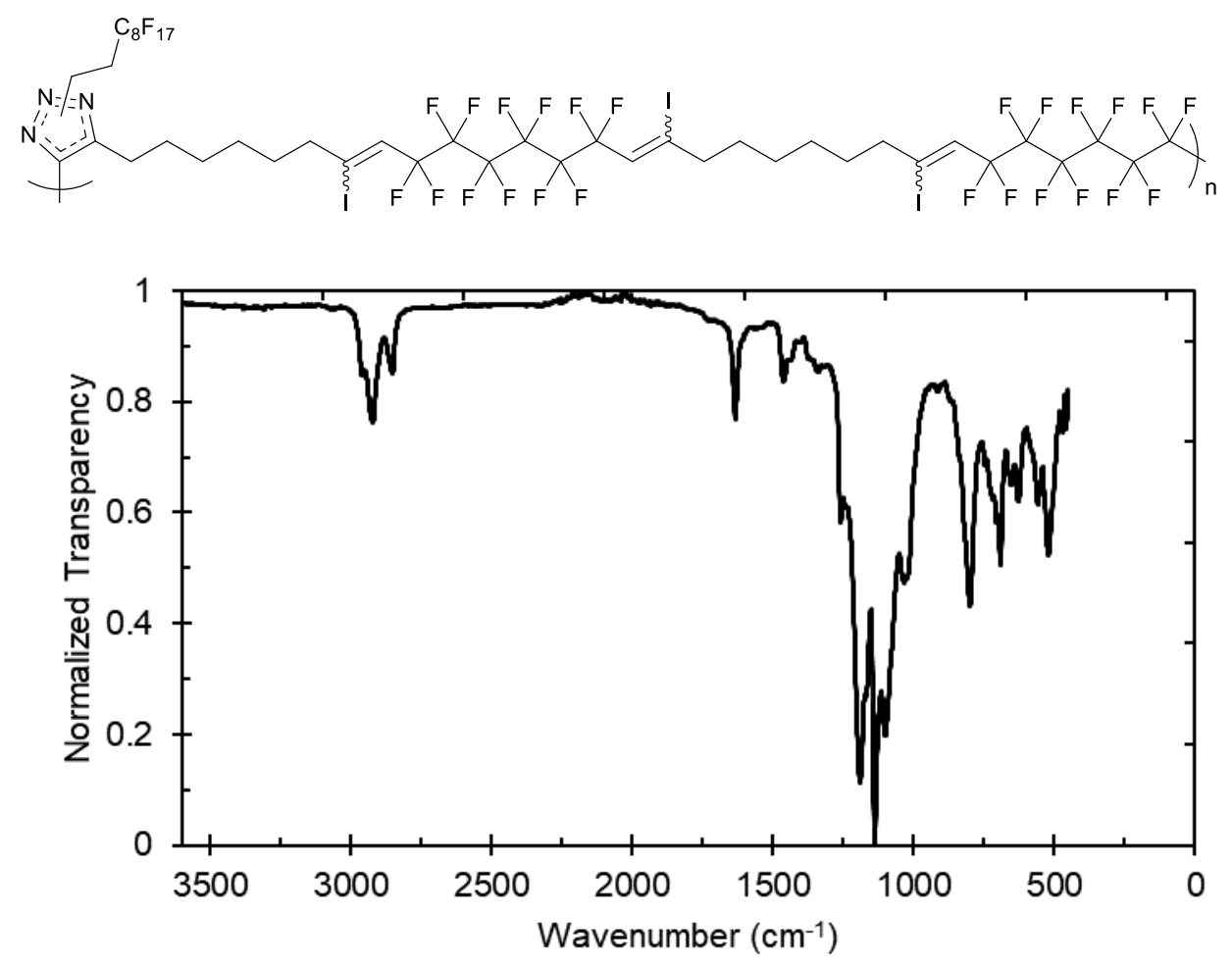


\section{GPC Traces:}

1-perfluorohexyl-2,9-diiodo-1,9-decadiene block polymer, 3, HPLC SEC:
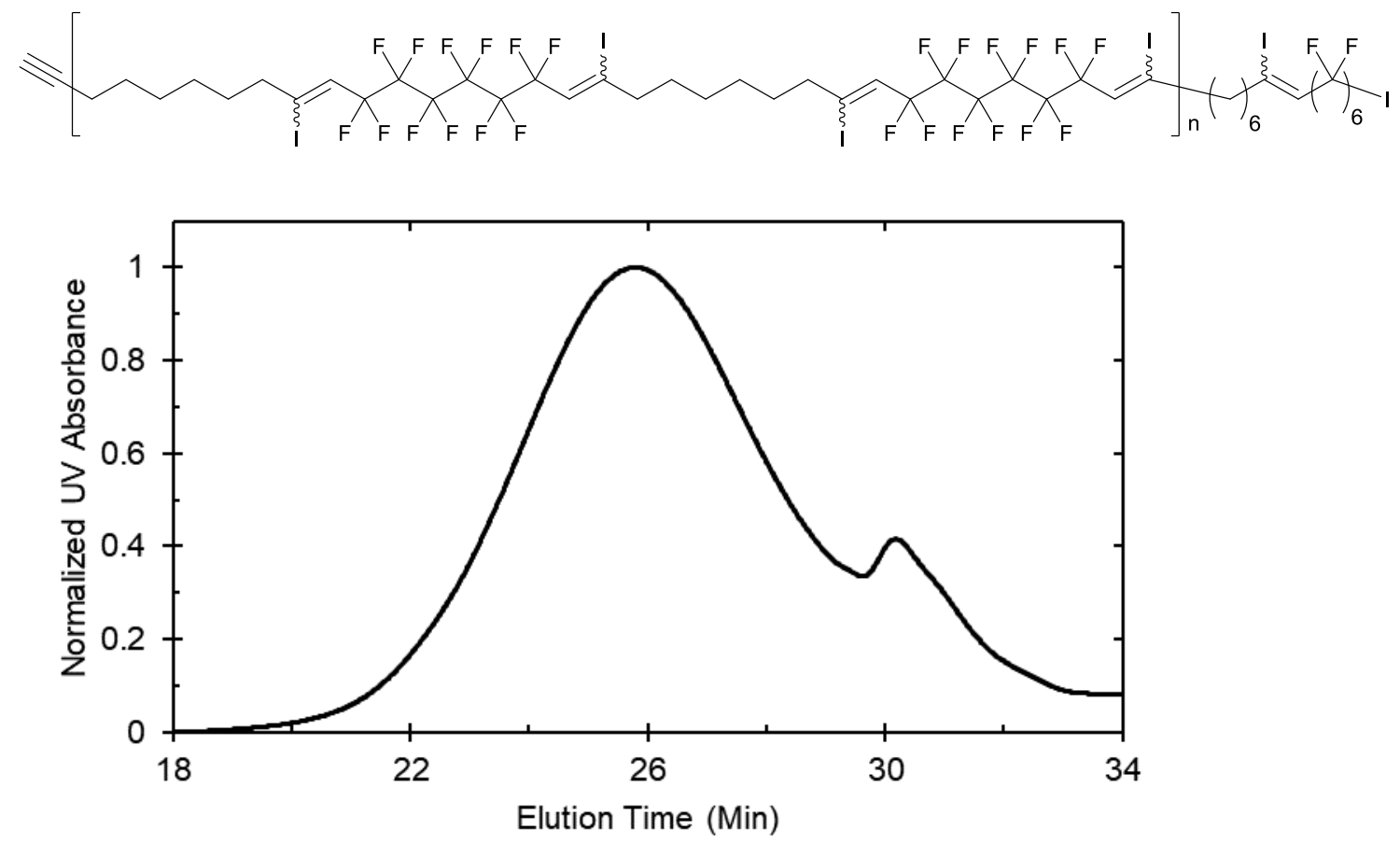

*We hypothesize that the low molecular shoulder is due to a small amount of oligomer or cyclic product that couldn't be removed by precipitation. 
1-perfluorohexyl-2,9-diiodo-1,9-decadiene block polymer, 3, UHPLC SEC, Table 1, Entry 1:
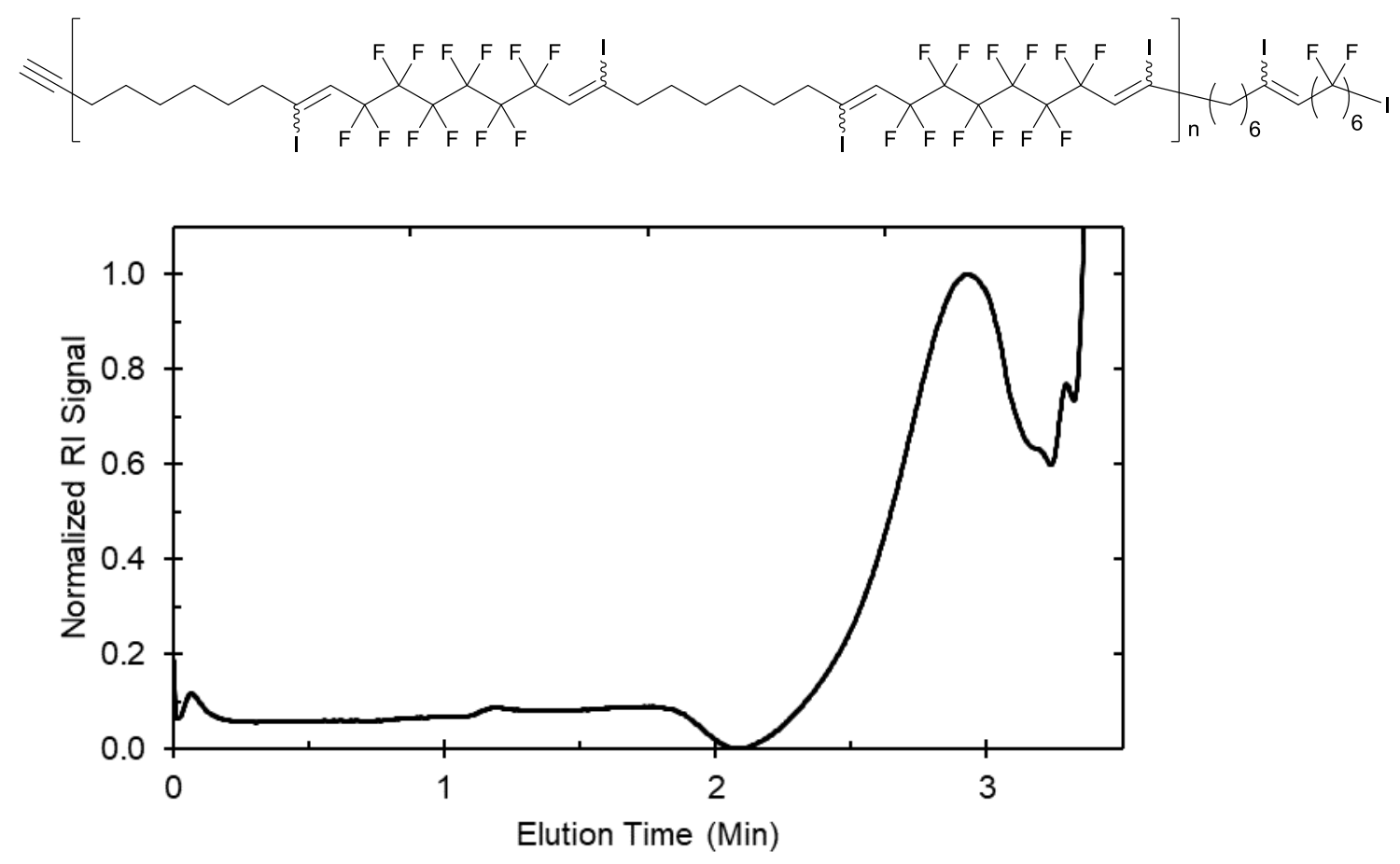

*Peak following 3.1 minutes belongs to solvent delay. 
1-perfluorohexyl-2,9-diiodo-1,9-decadiene block polymer, 3, UHPLC SEC, Table 1, Entry 2
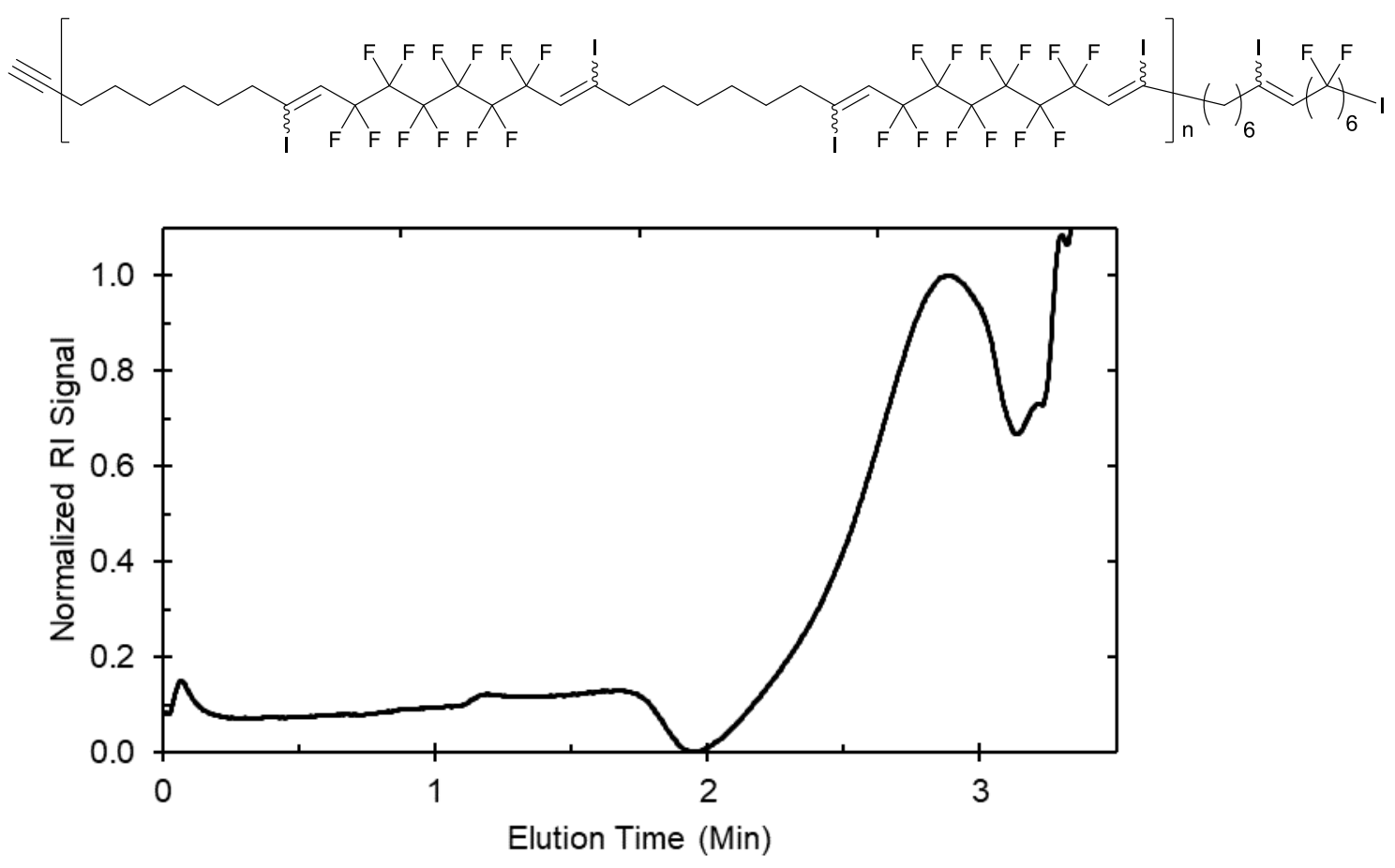

*Peak following 3.1 minutes belongs to solvent delay. 
1-perfluorohexyl-2,9-diiodo-1,9-decadiene block polymer, 3, UHPLC SEC, Table 1, Entry 3:
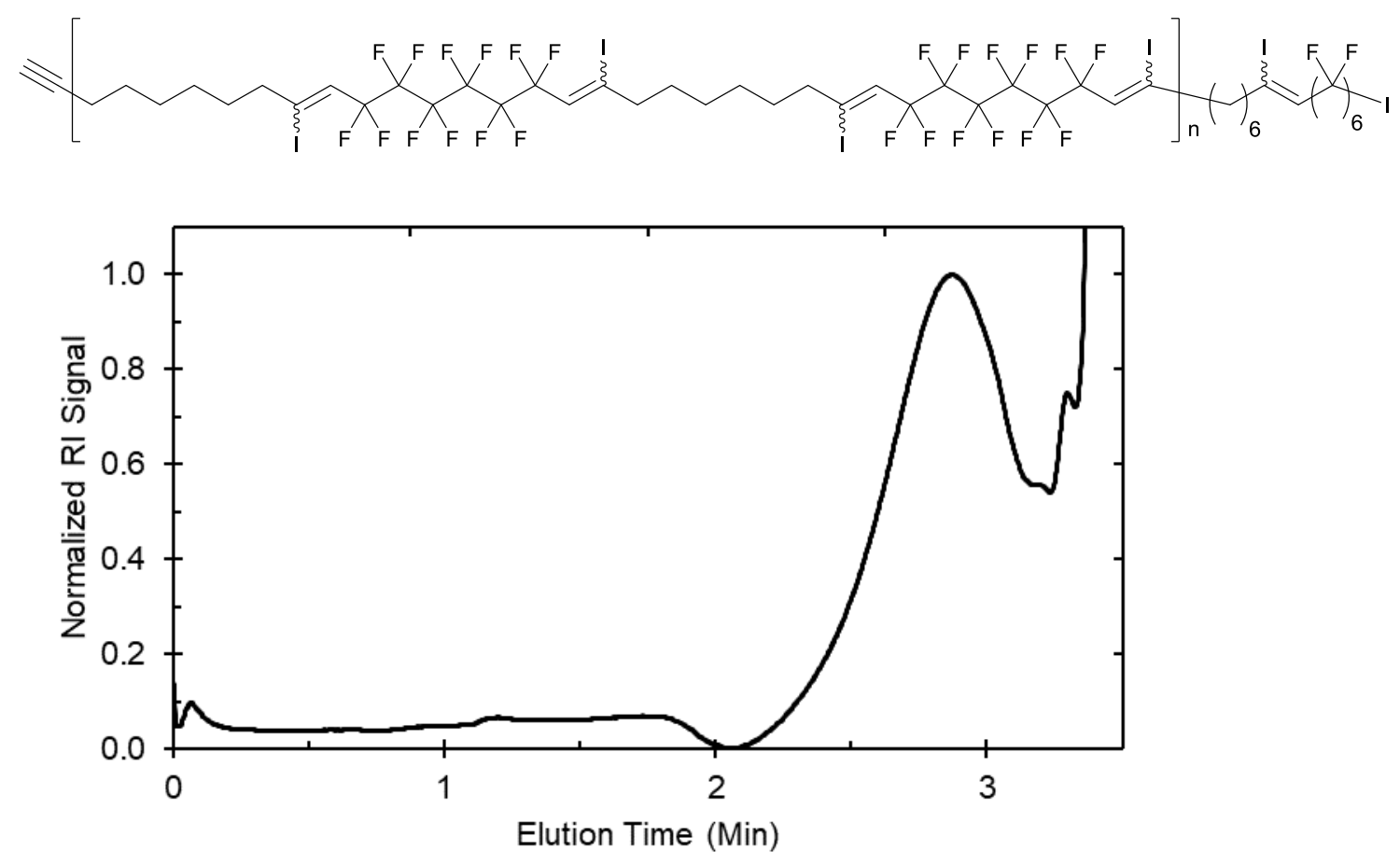

*Peak following 3.1 minutes belongs to solvent delay. 
1-perfluorohexyl-2,9-diiodo-1,9-decadiene block polymer, 3, UHPLC SEC, Table 1, Entry 4:
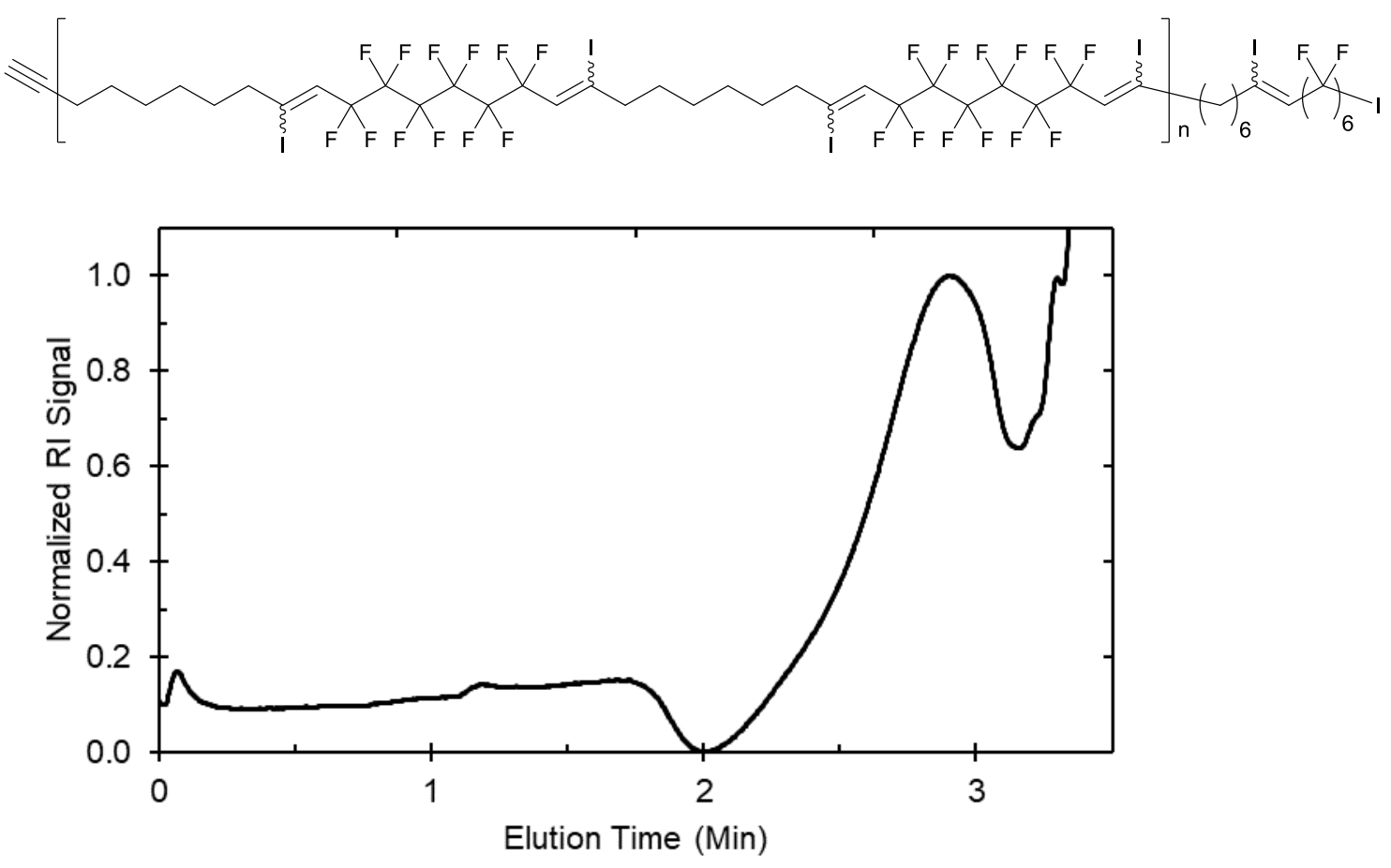

*Peak following 3.1 minutes belongs to solvent delay. 
1-perfluorohexyl-2,9-diiodo-1,9-decadiene block polymer, 3, UHPLC SEC, Table 1, Entry 5:
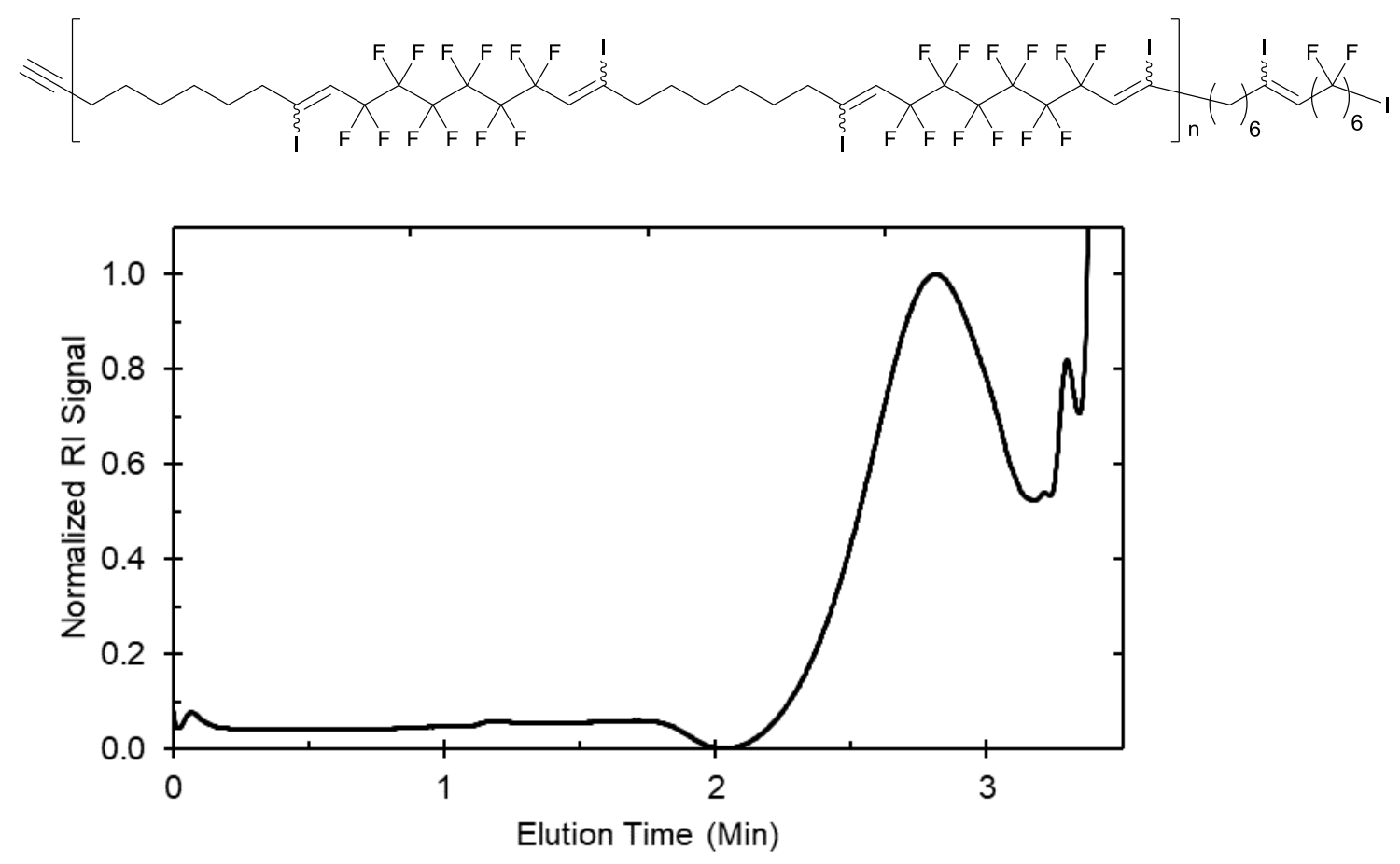

*Peak following 3.1 minutes belongs to solvent delay. 
1-perfluorohexyl-2,9-diiodo-1,9-decadiene block polymer, 3, UHPLC SEC, Table 1, Entry 6:
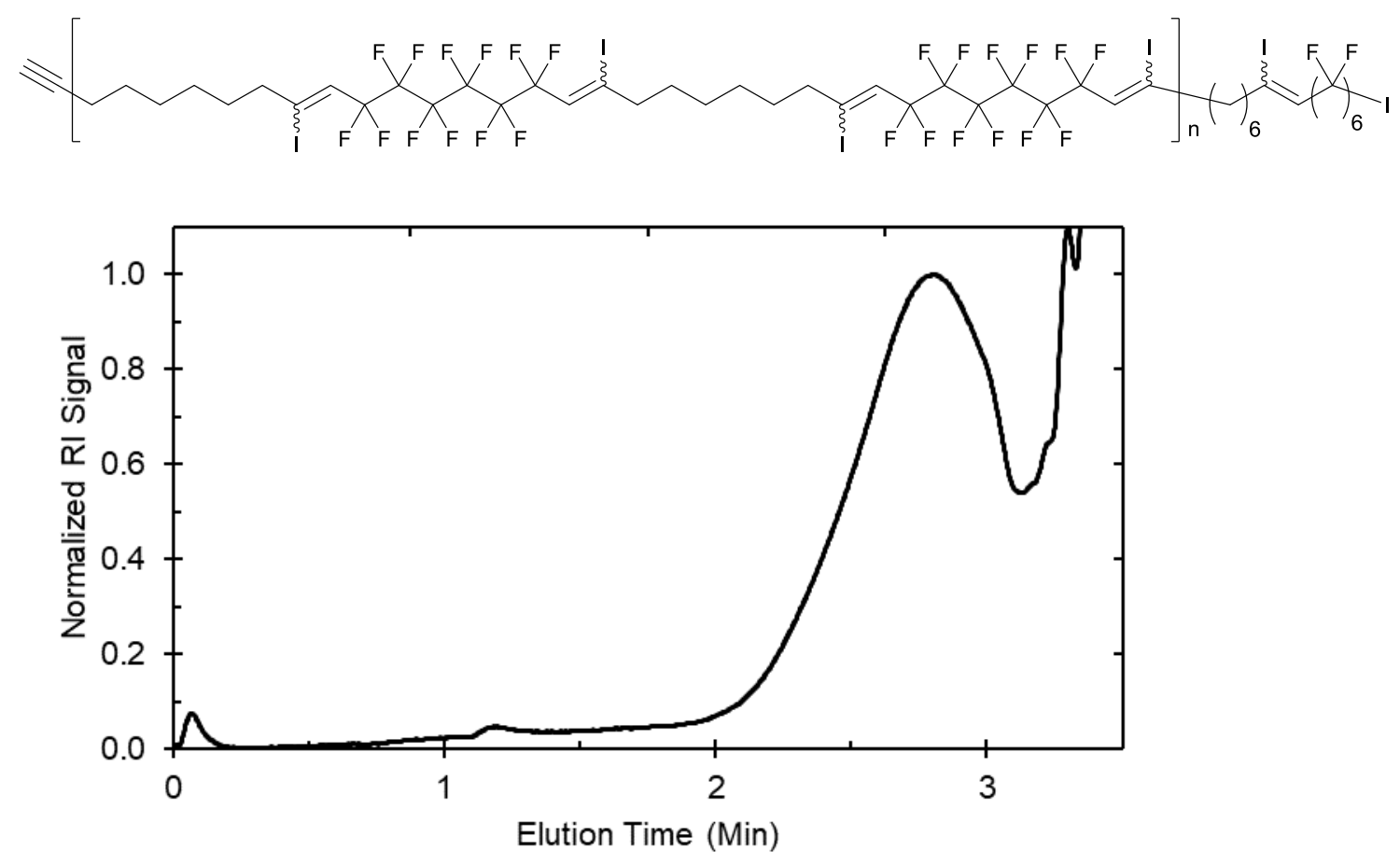

*Peak following 3.1 minutes belongs to solvent delay. 
1-perfluorohexyl-2,9-diiodo-1,9-decadiene block polymer, 3, UHPLC SEC, Table 1, Entry 7:
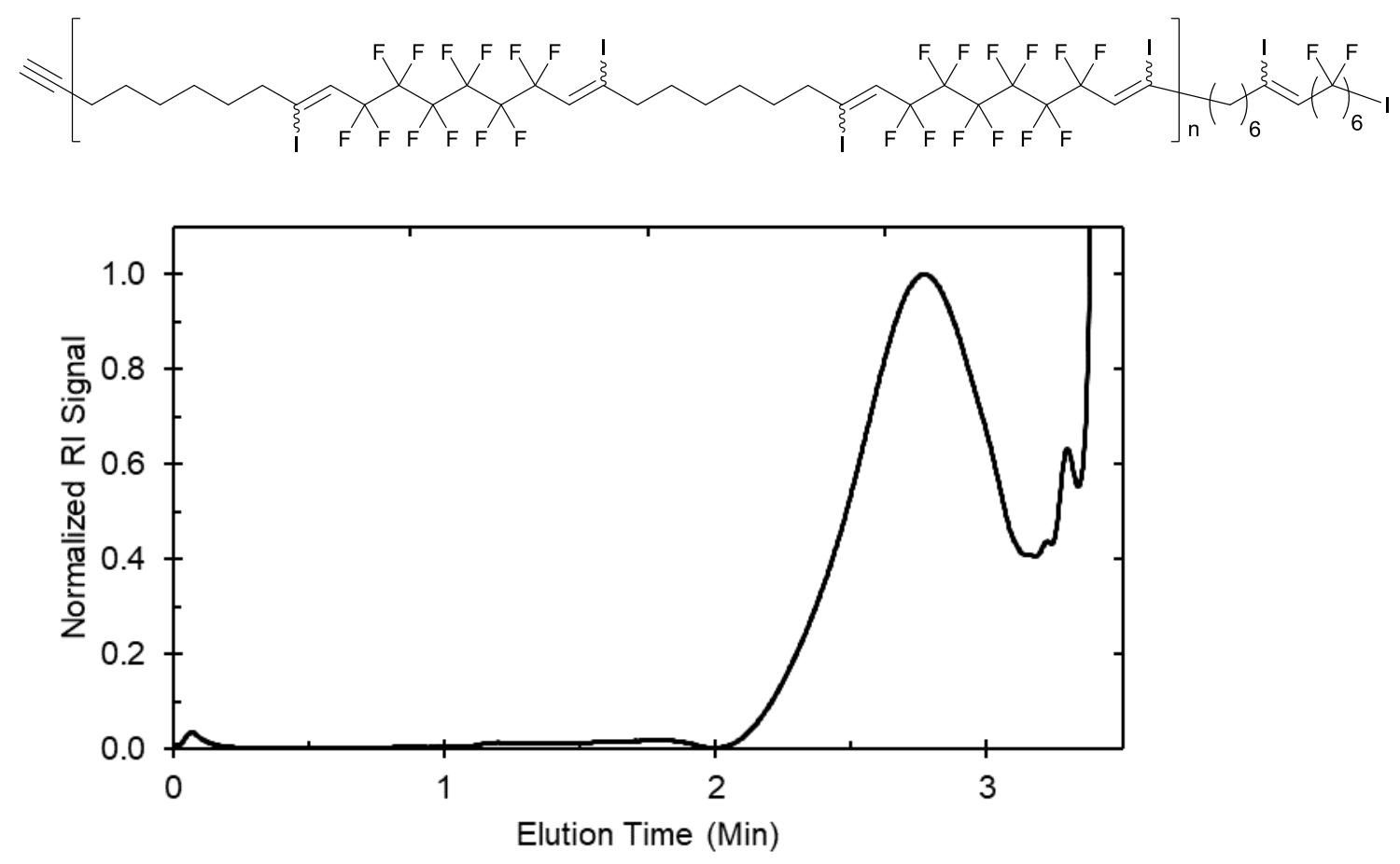

*Peak following 3.1 minutes belongs to solvent delay. 
1-perfluorohexyl-2,9-diiodo-1,9-decadiene block polymer, 3, UHPLC SEC, Table 1, Entry 8:
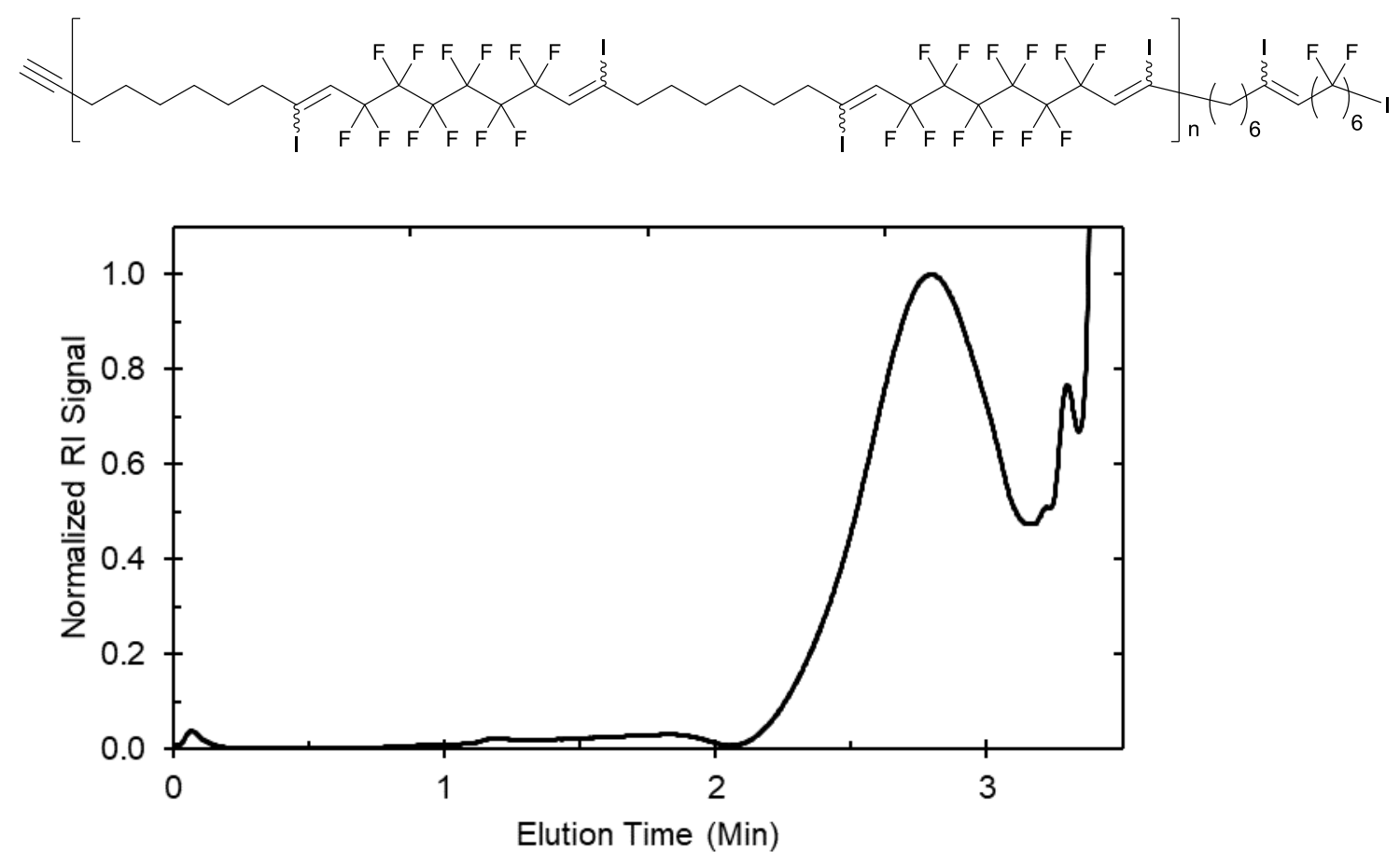

*Peak following 3.1 minutes belongs to solvent delay. 
1-perfluorohexyl-2,9-diiodo-1,9-decadiene block polymer, 3, UHPLC SEC, Table 1, Entry 1:
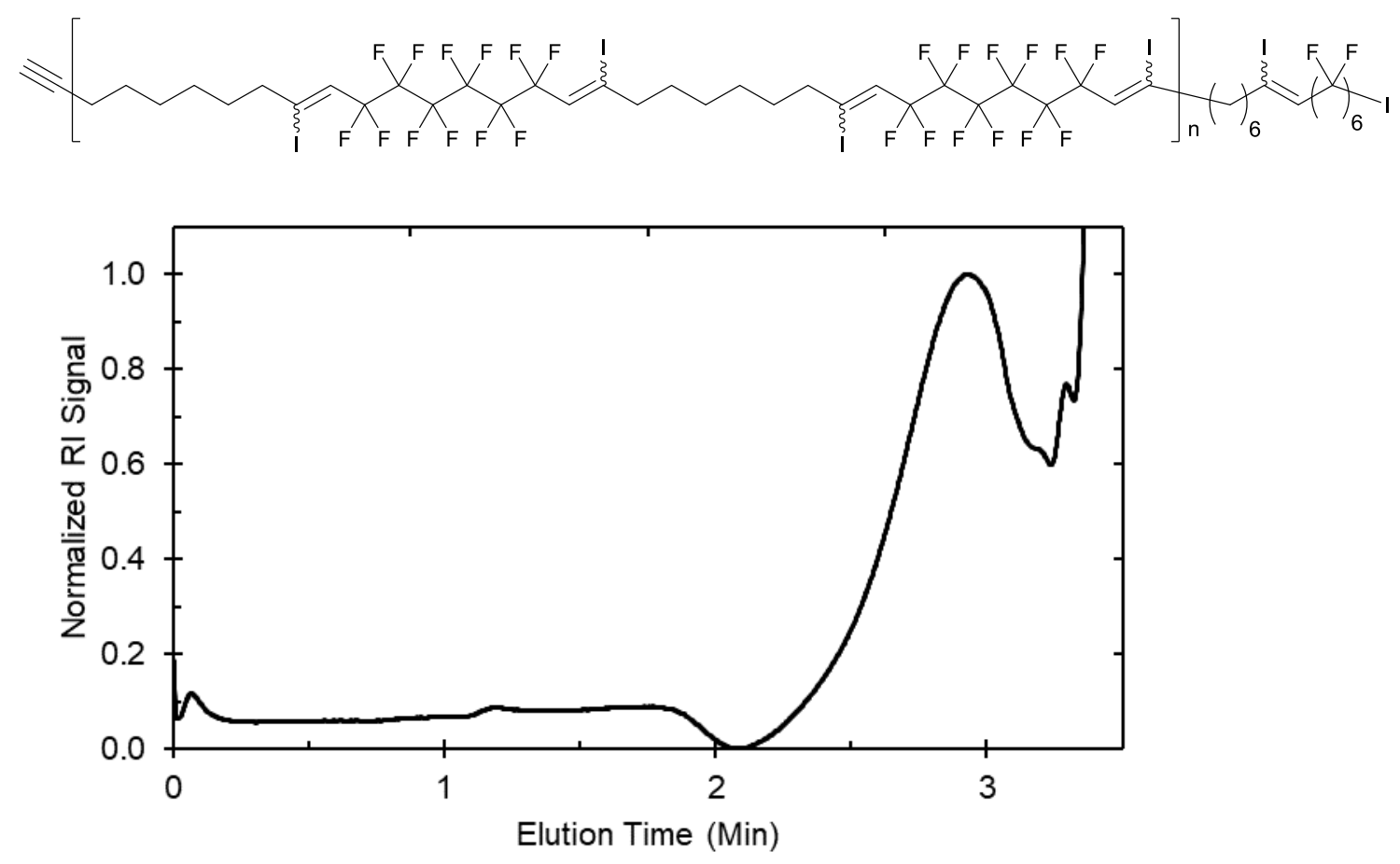

*Peak following 3.1 minutes belongs to solvent delay. 
Thiol capping of polymer 3,4 :
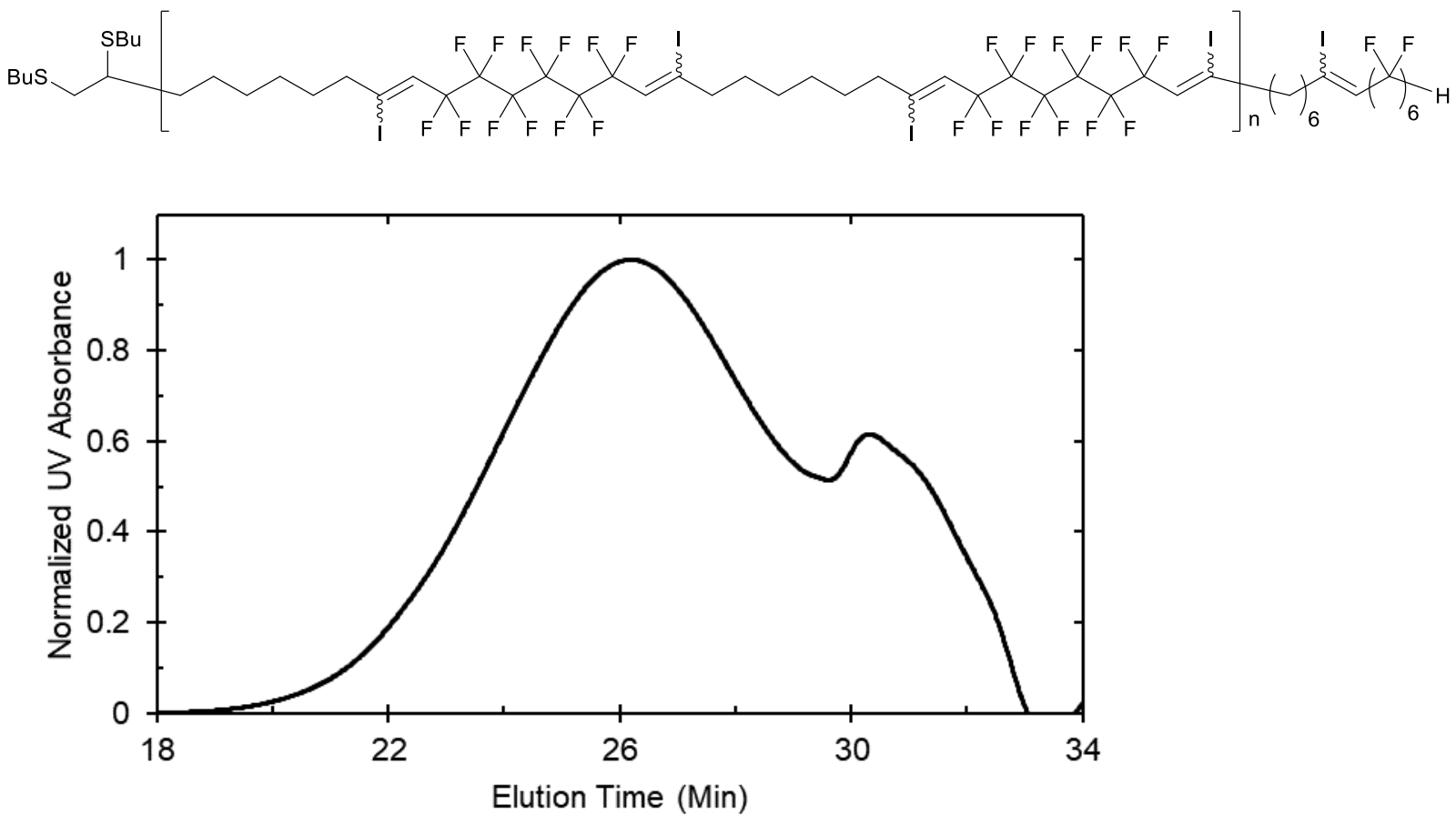
Sonogashira coupling of polymer 4, 5 :
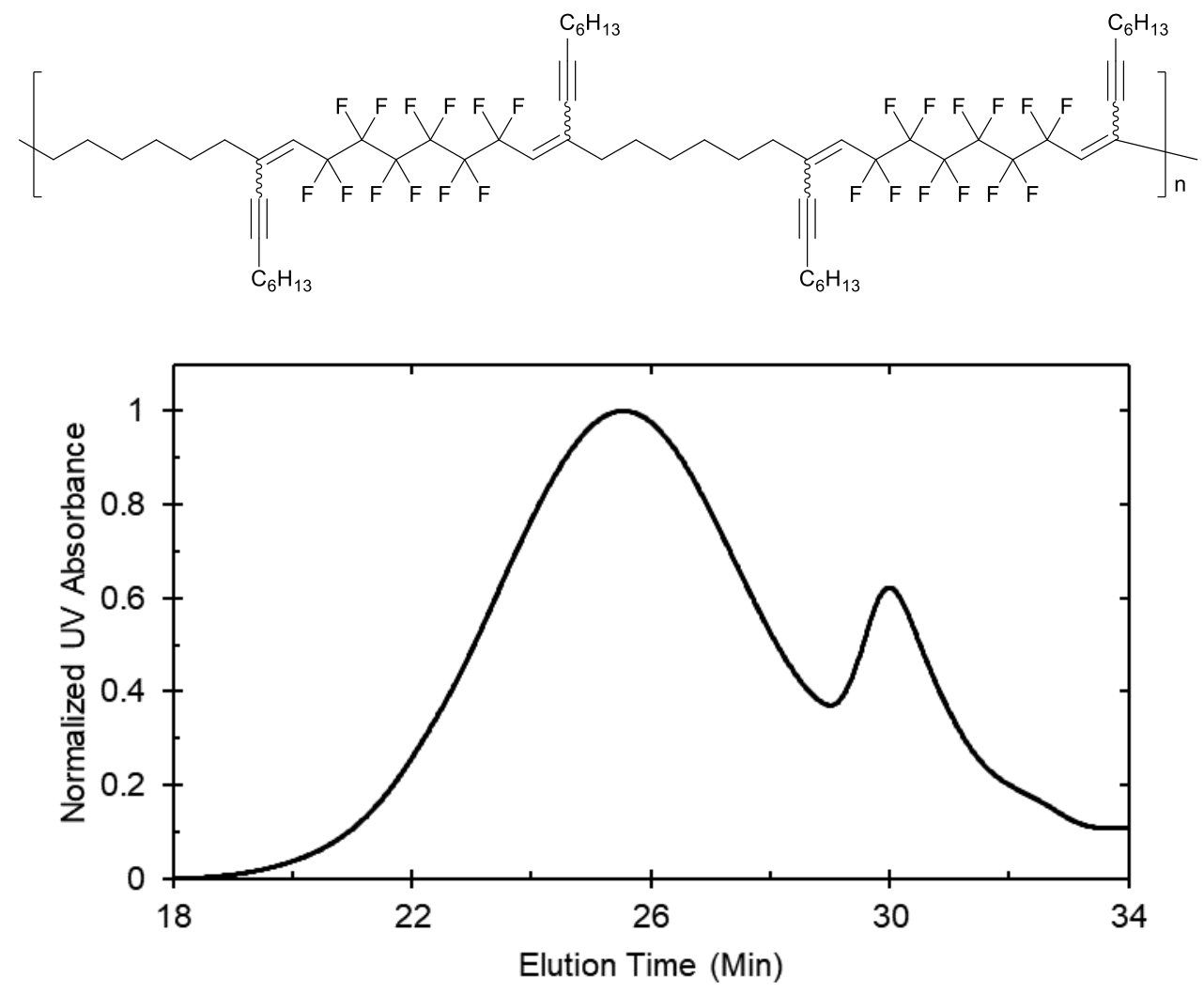
Stille coupling of polymer 4,6 :
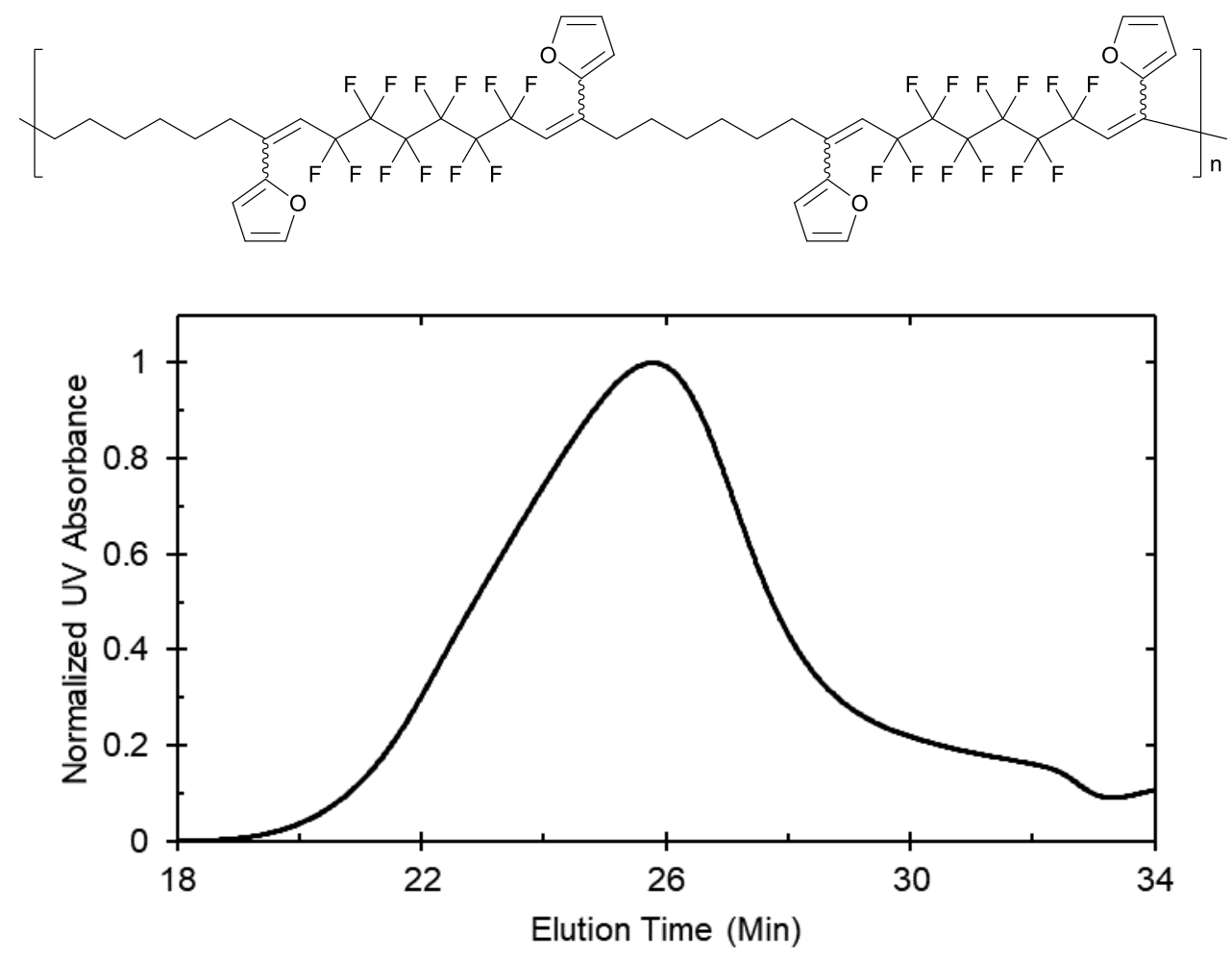
Suzuki coupling of polymer 4,7 :
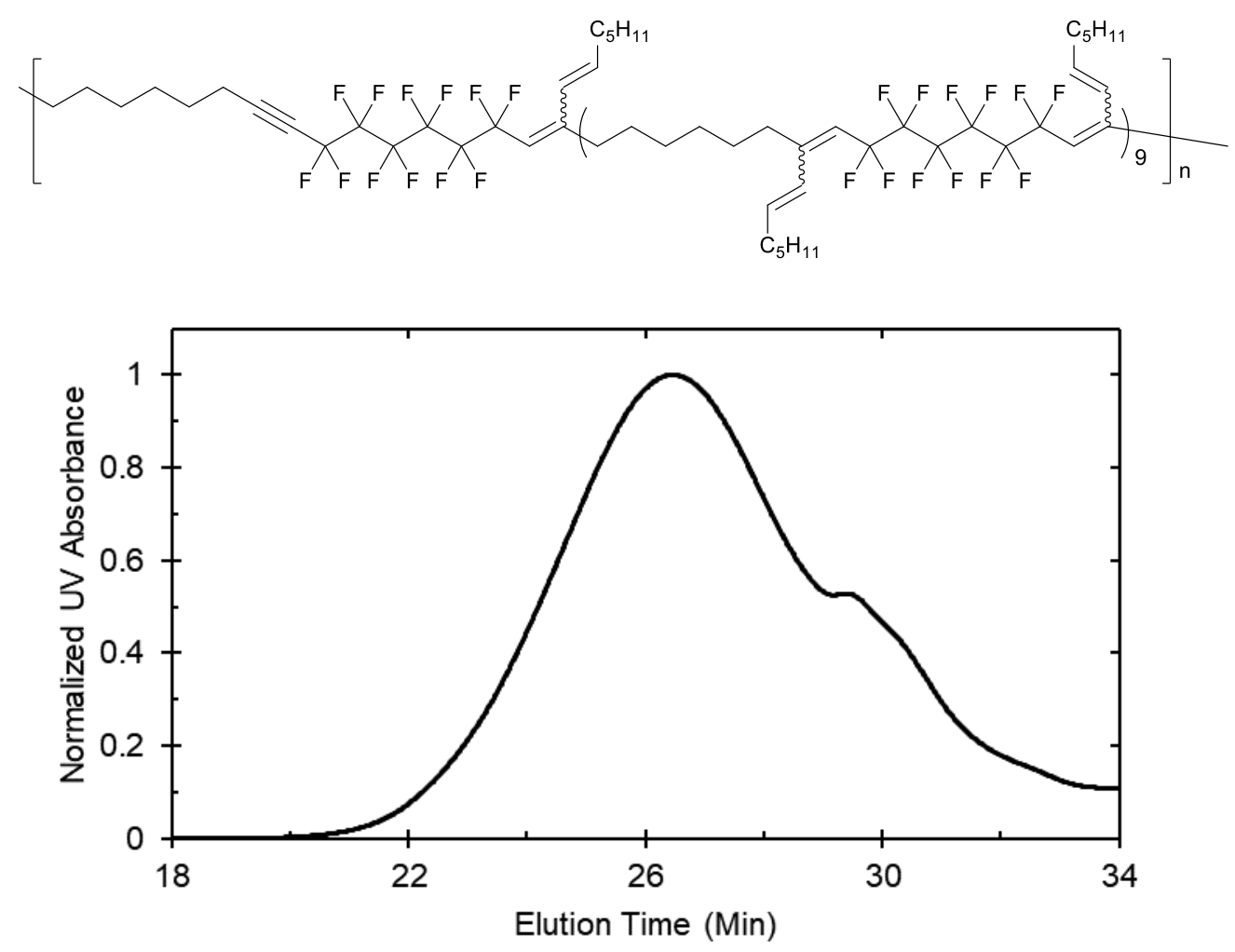
Phenol coupling of polymer 4, 8:
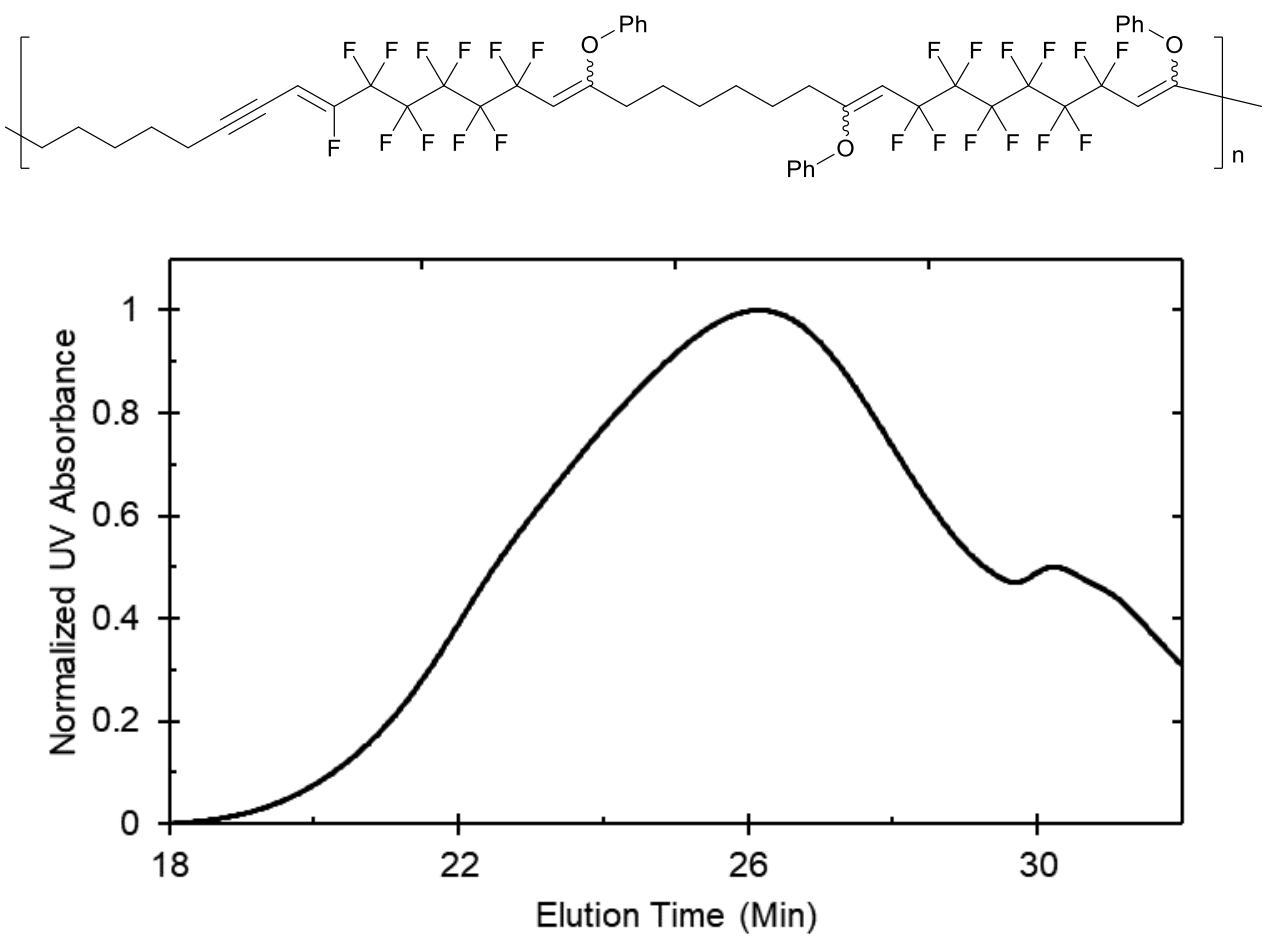
Thiophenol coupling of polymer 4, 9:
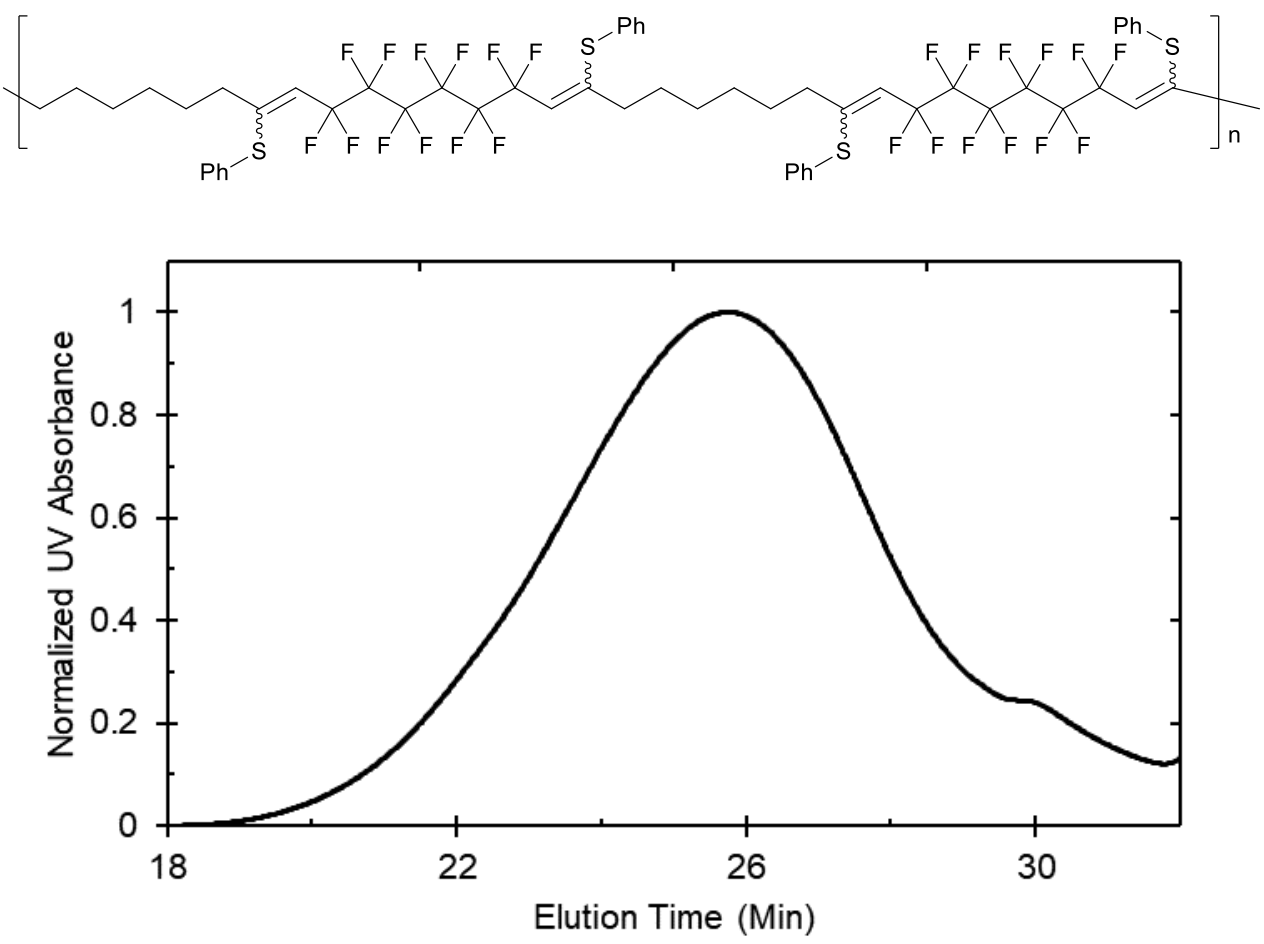
Kumada coupling of polymer 3,10 :
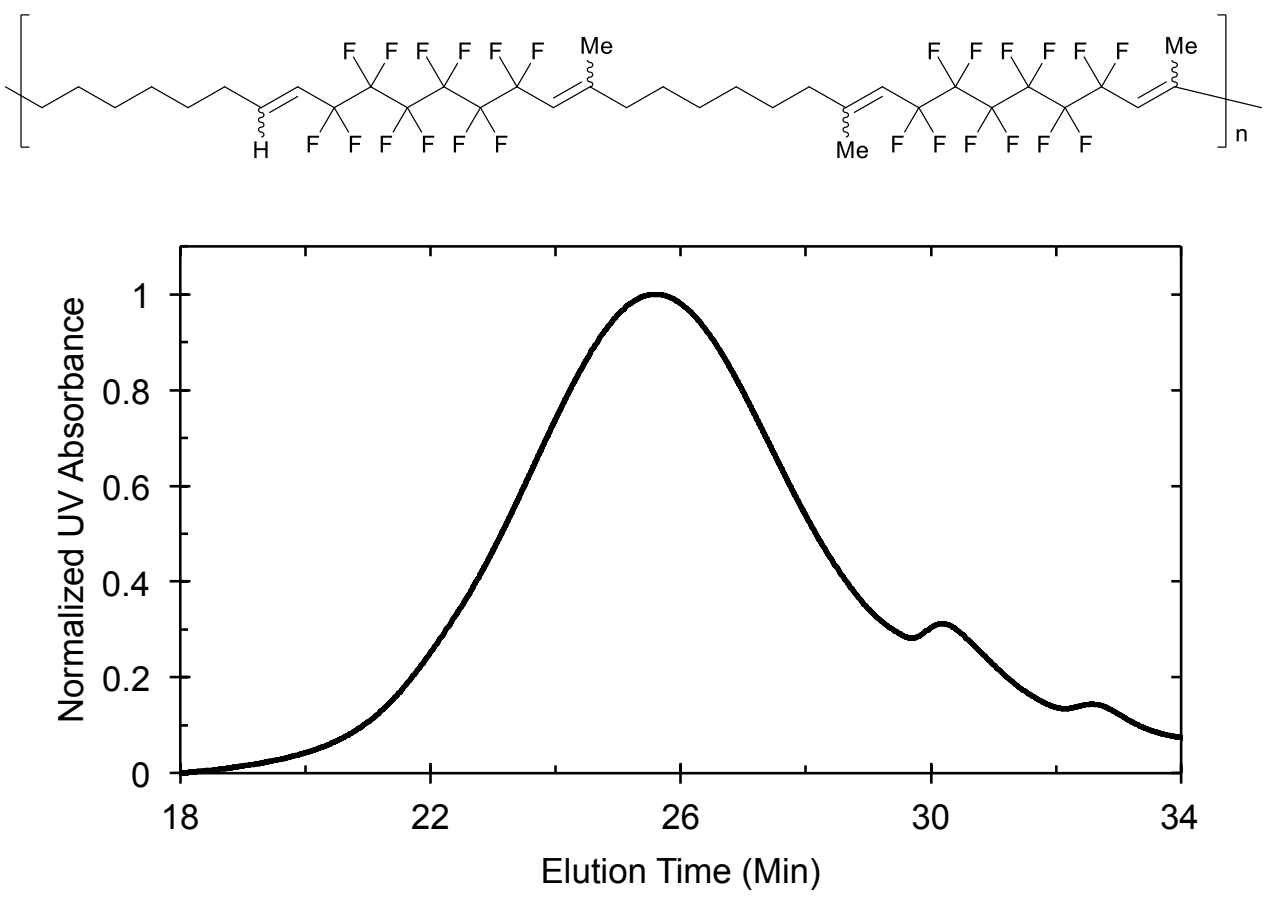


\section{TGA Traces:}

1-perfluorohexyl-2,9-diiodo-1,9-decadiene block polymer, 3:
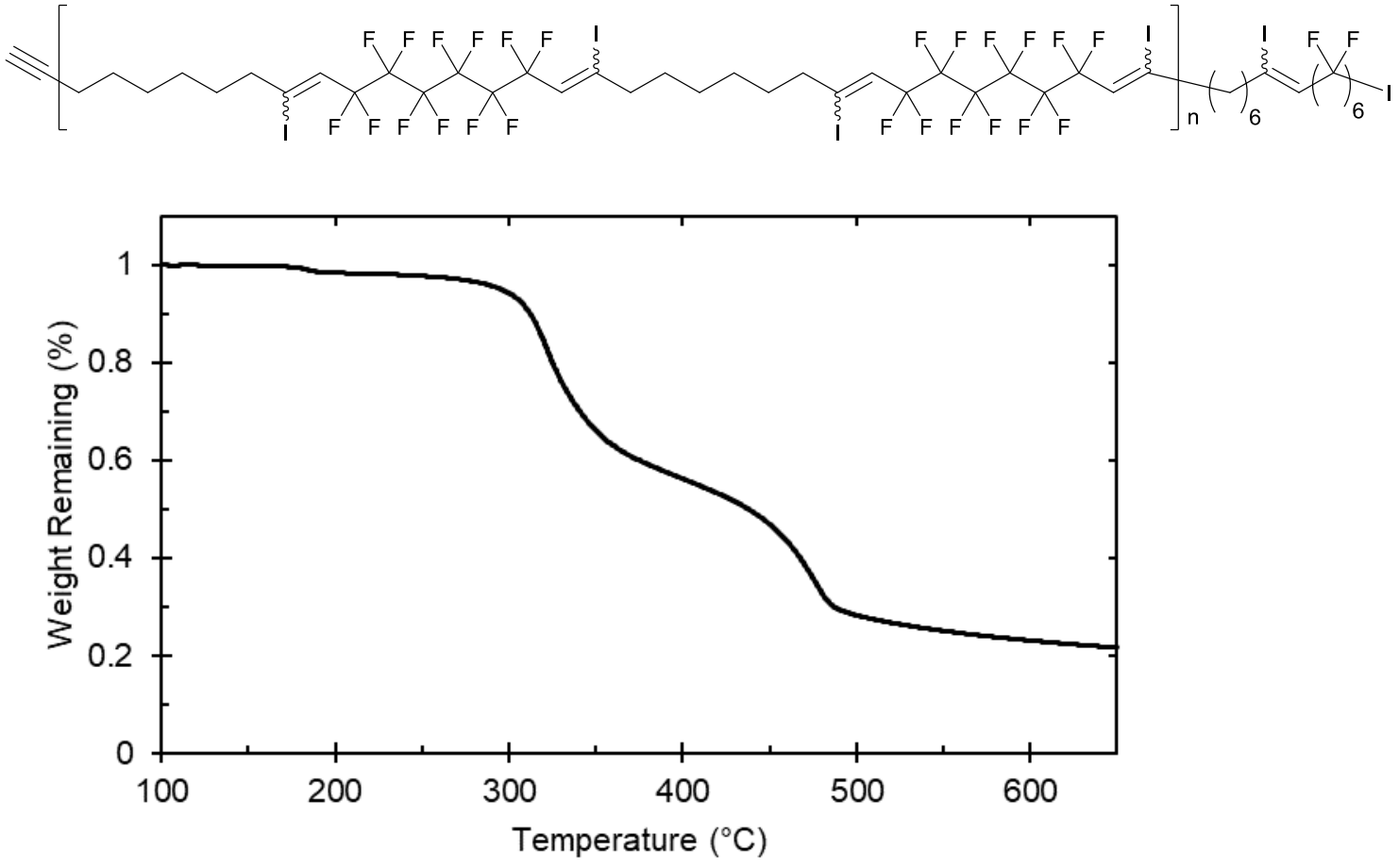
Sonogashira coupling of polymer 4, 5 :
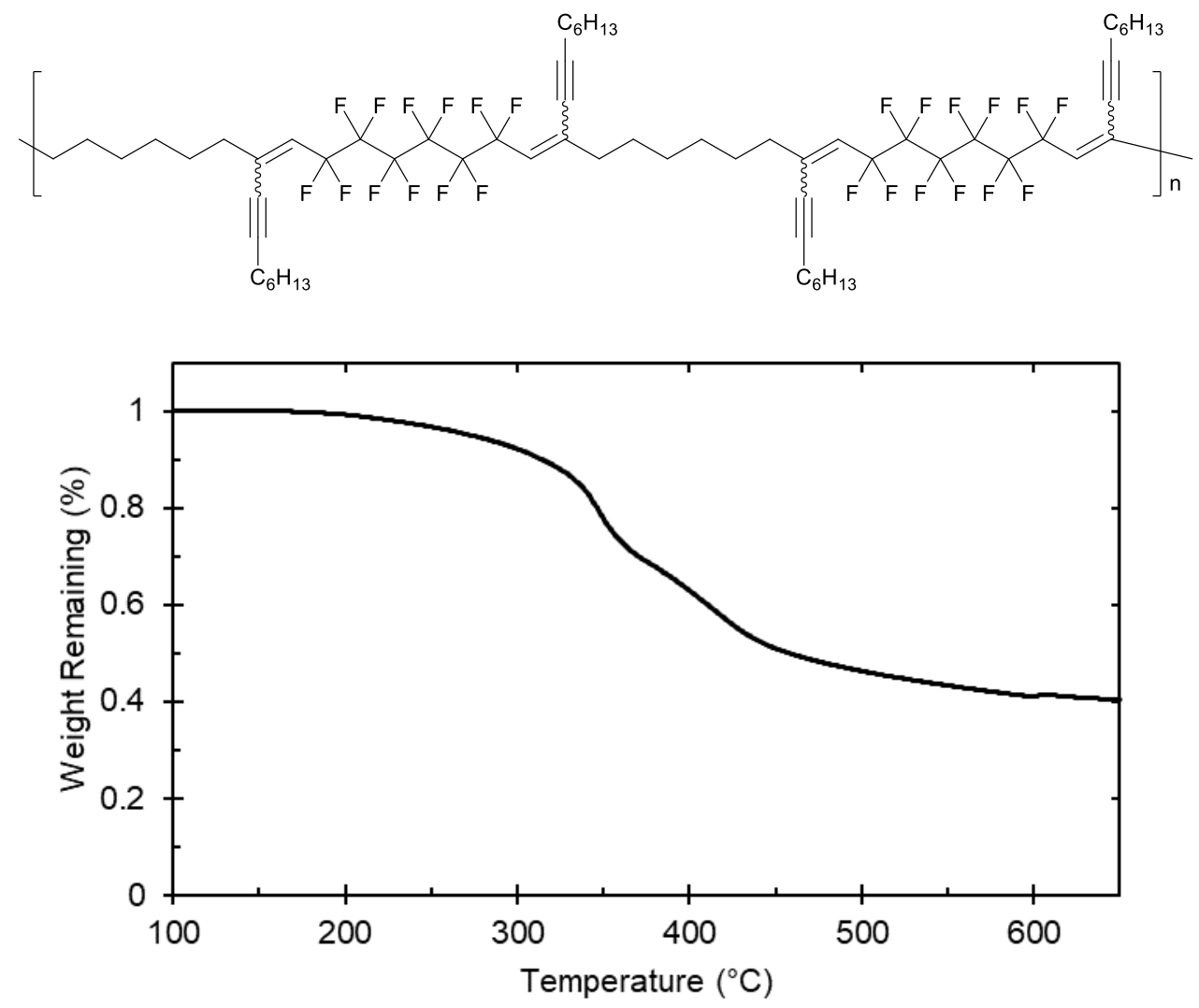
Stille coupling of polymer 4,6 :
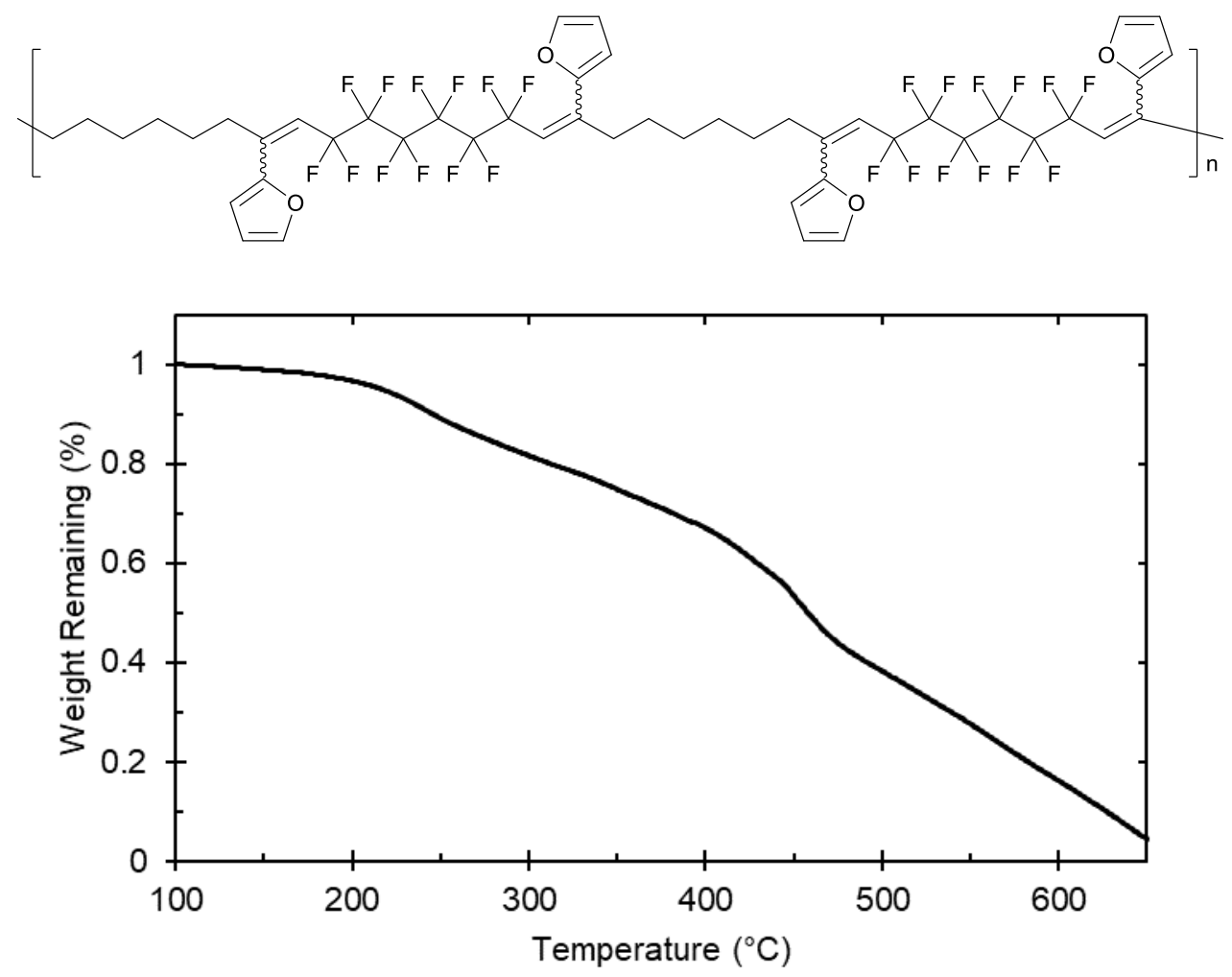
Suzuki coupling of polymer 4,7 :
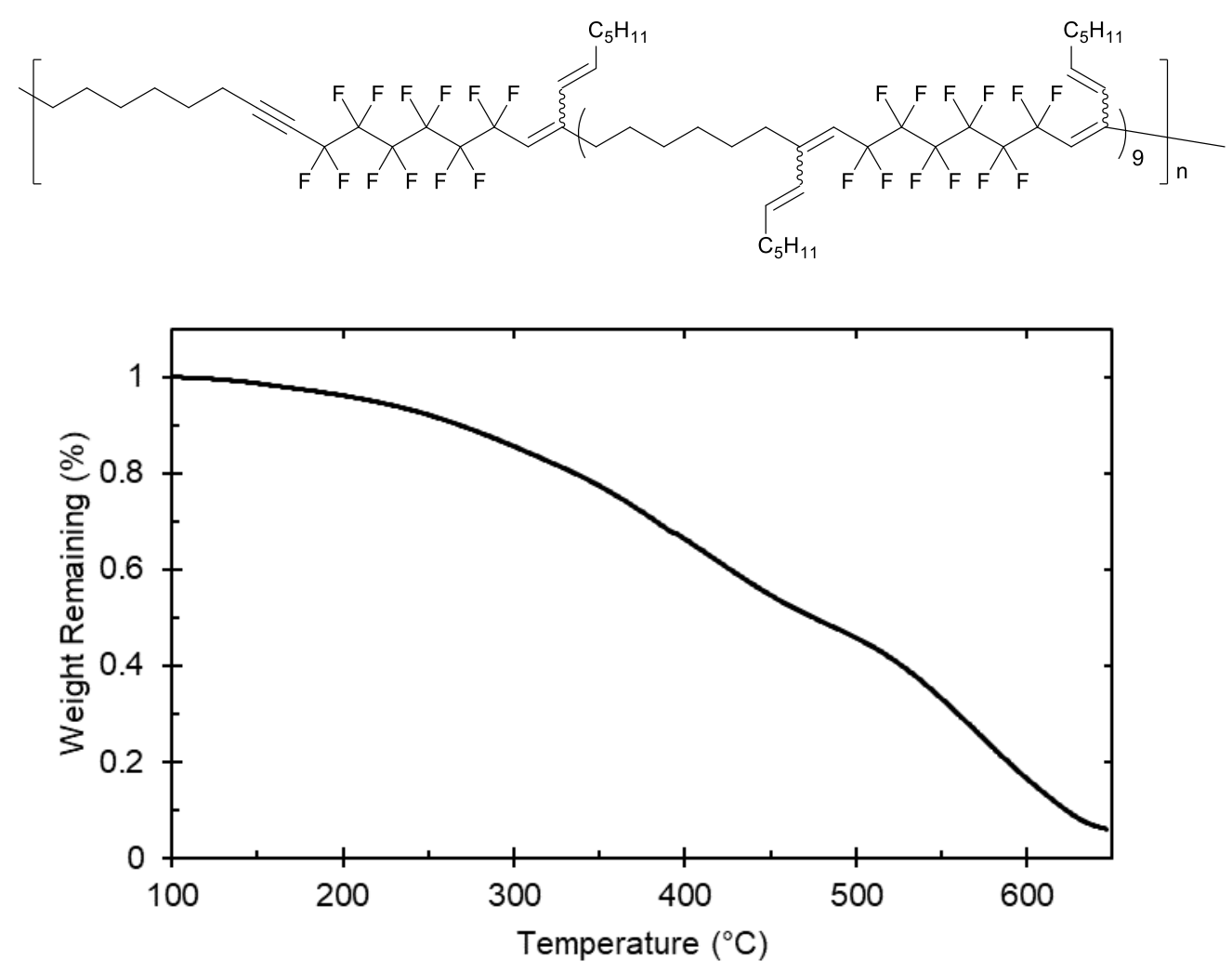
Phenol coupling of polymer 4, 8:
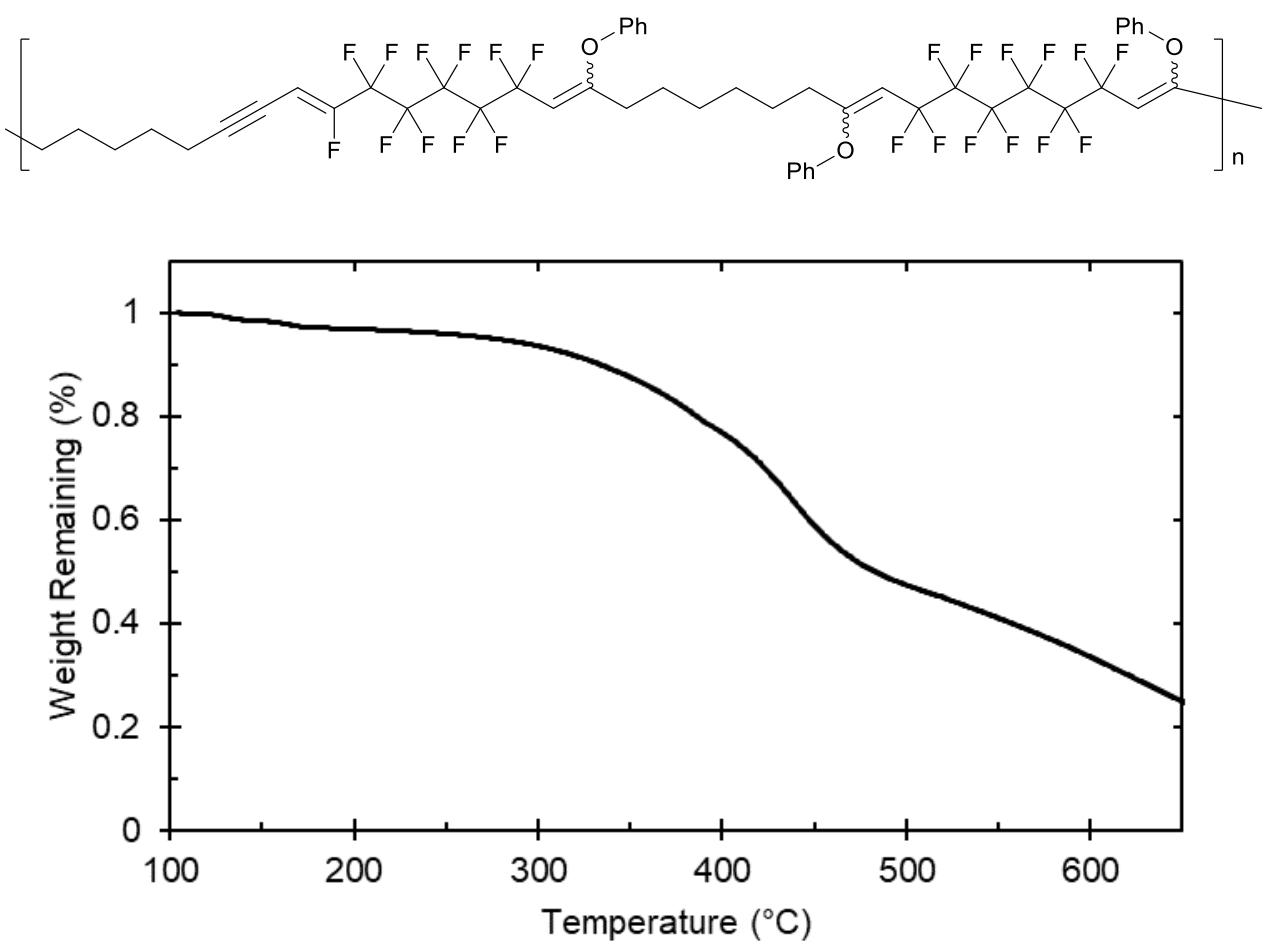
Thiophenol coupling of polymer 4, 9:
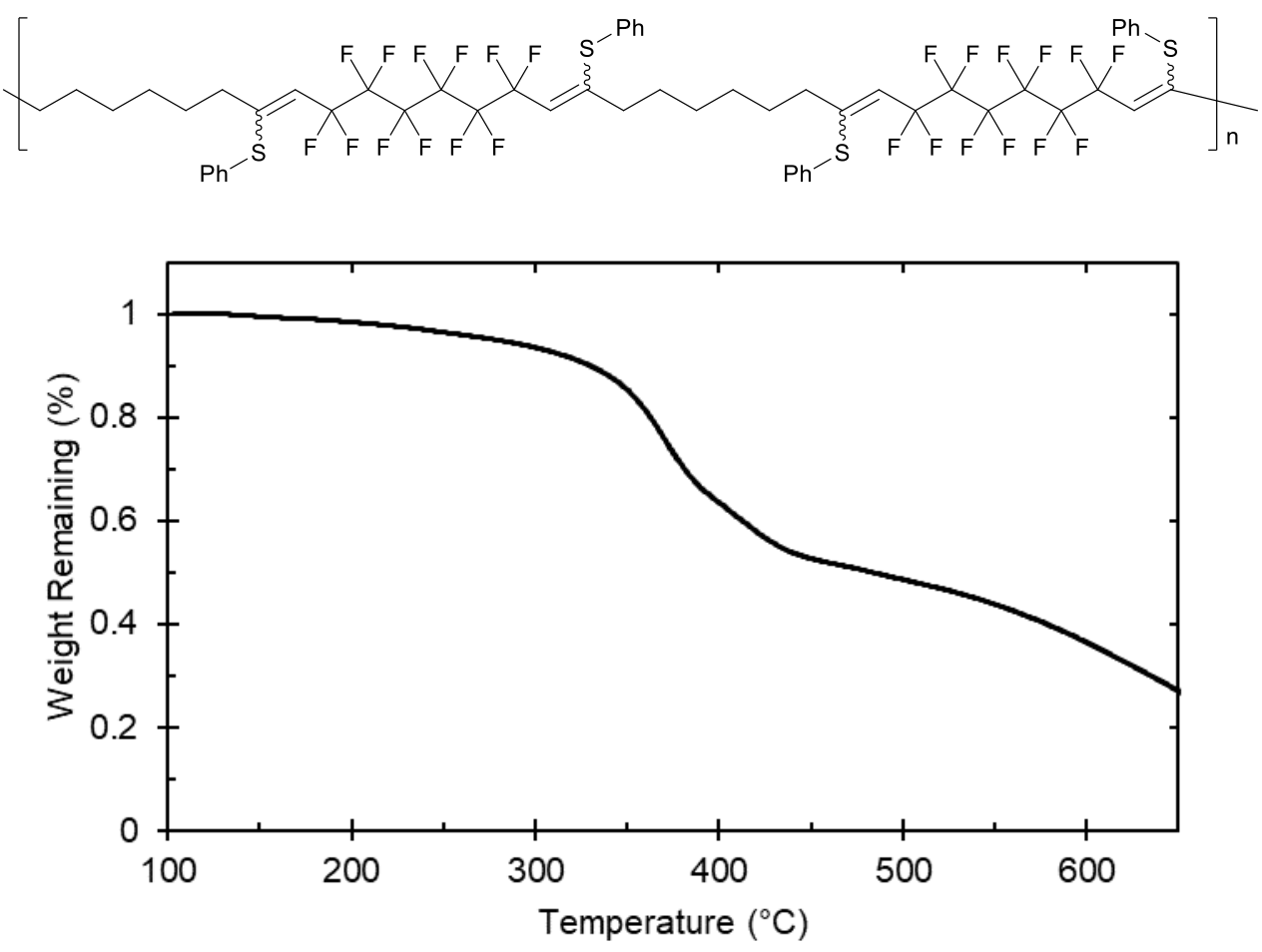
Kumada coupling of polymer 3,10 :
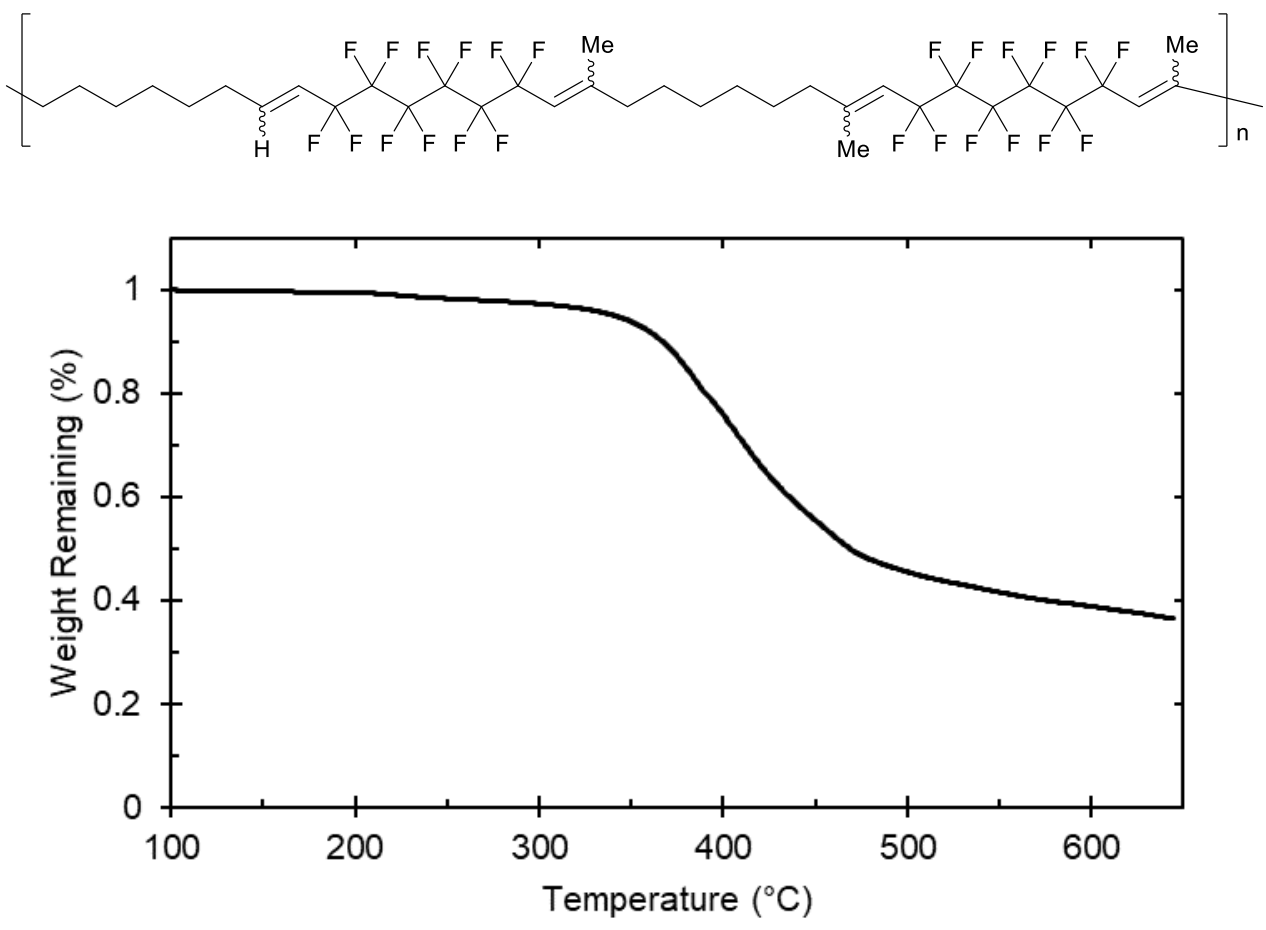
Elimination of iodide from polymer 3,11 :
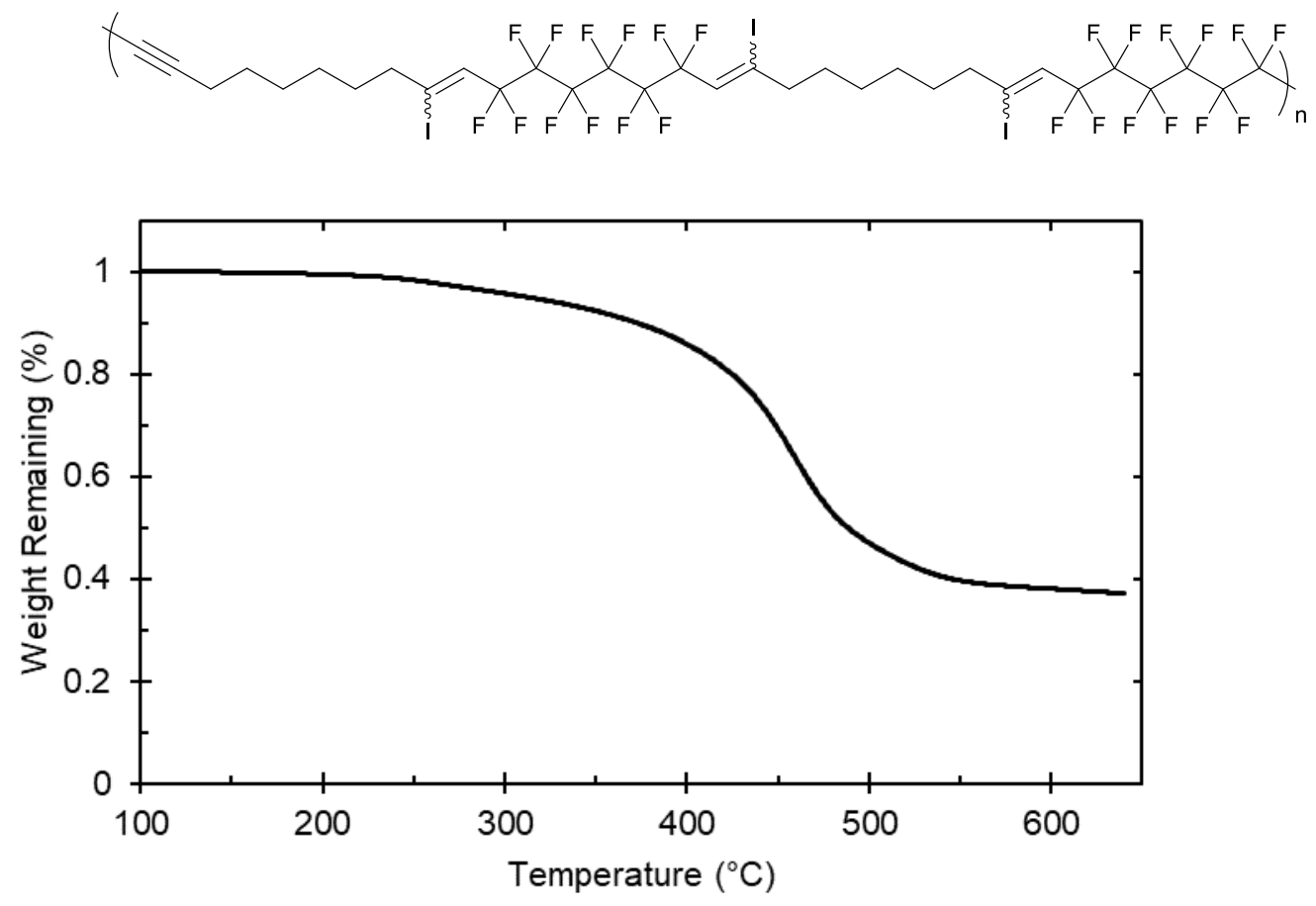
DSC Traces:

Sonogashira coupling of polymer 4,5 :
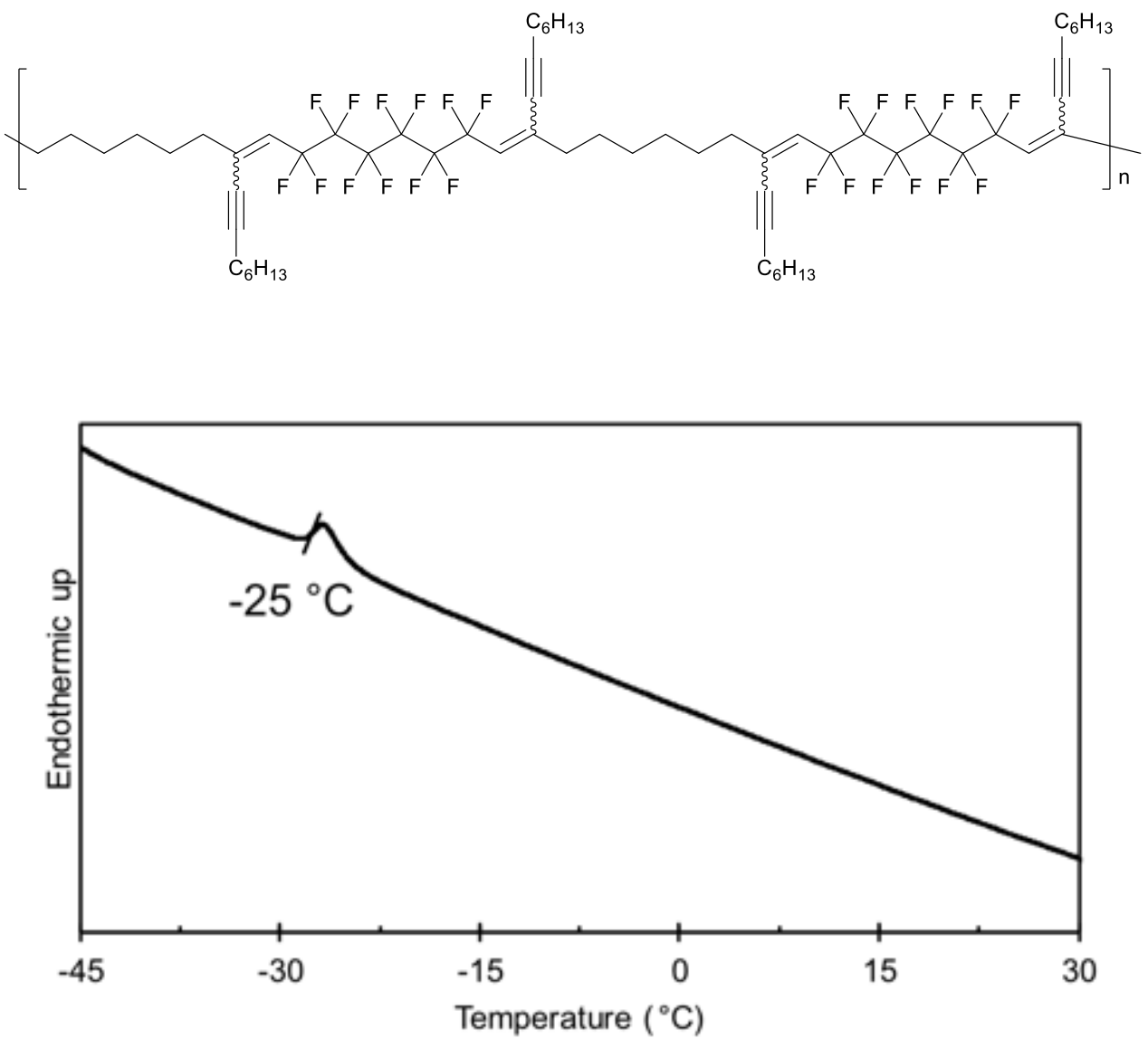
Stille coupling of polymer 4,6 :
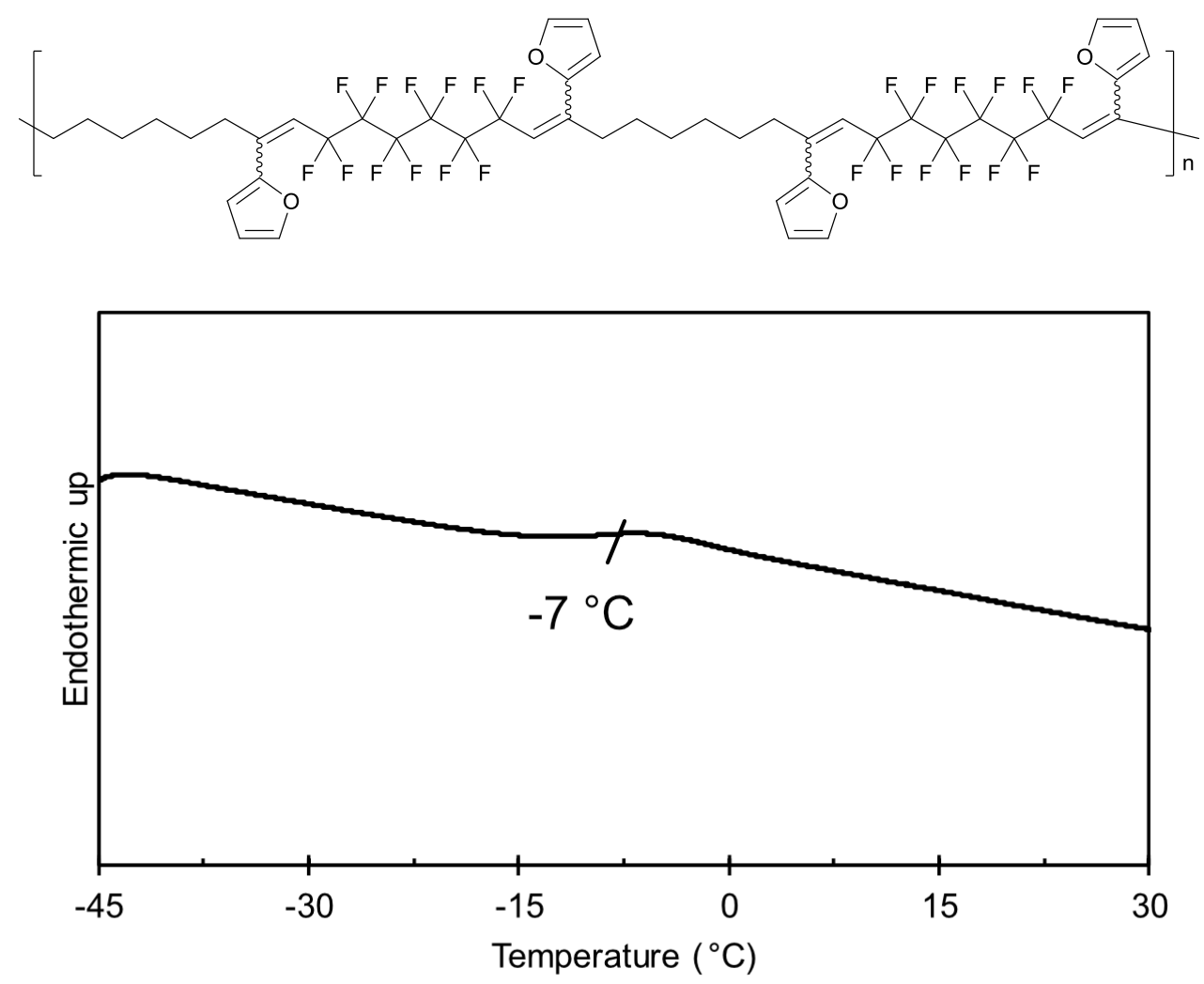
Suzuki coupling of polymer 4, 7:
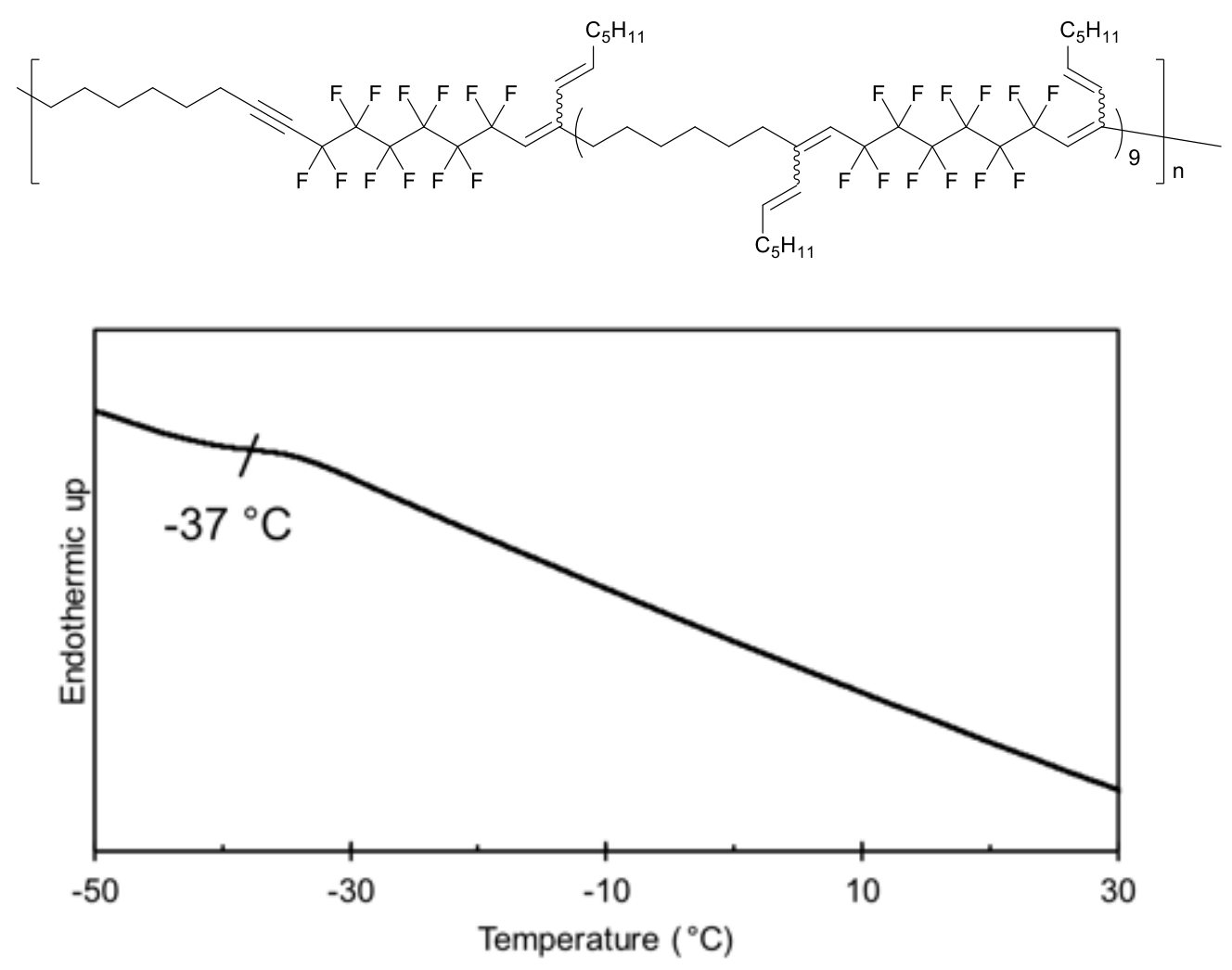
Phenol coupling of polymer 4, 8:
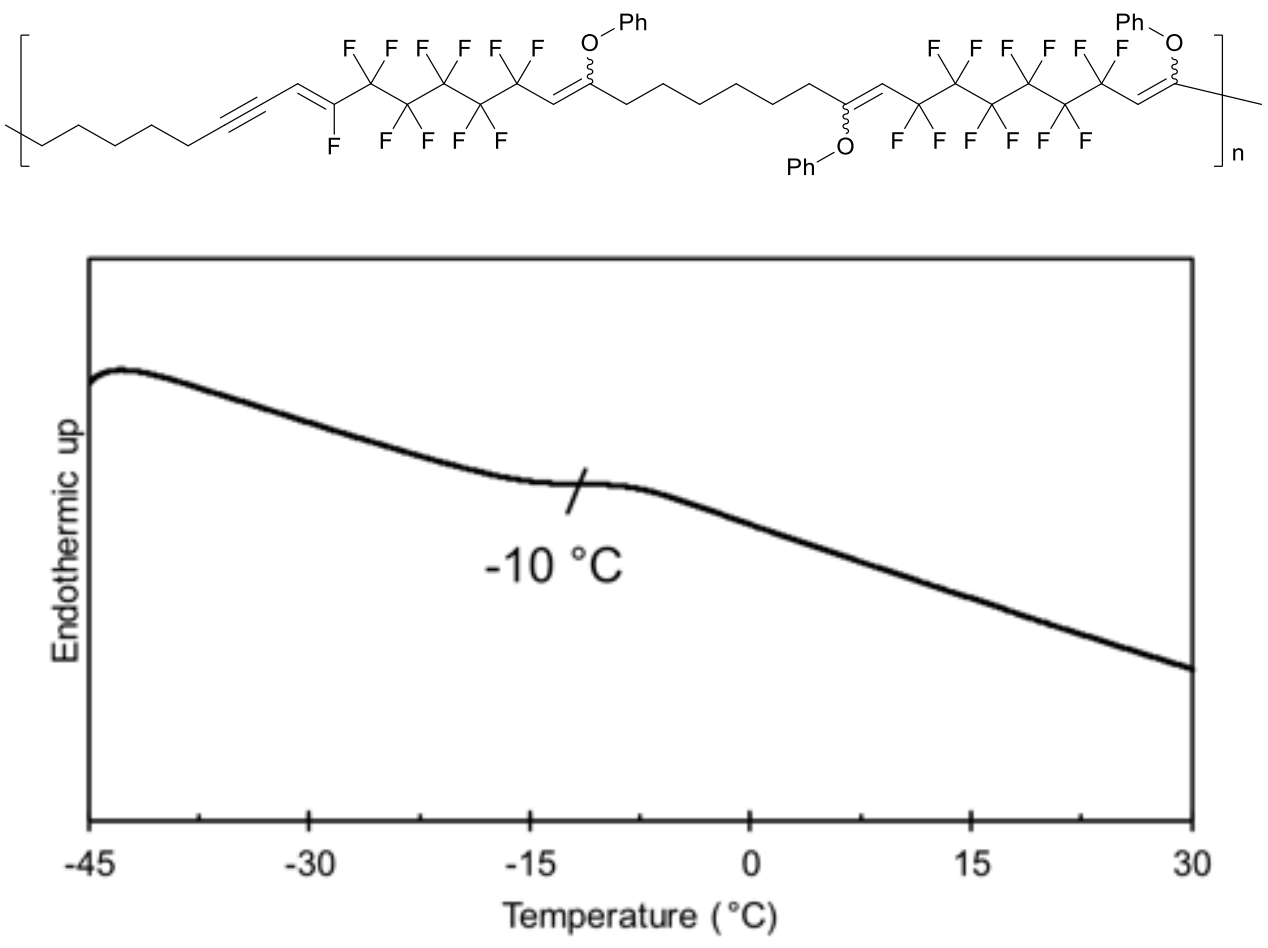
Thiophenol coupling of polymer 4, 9:
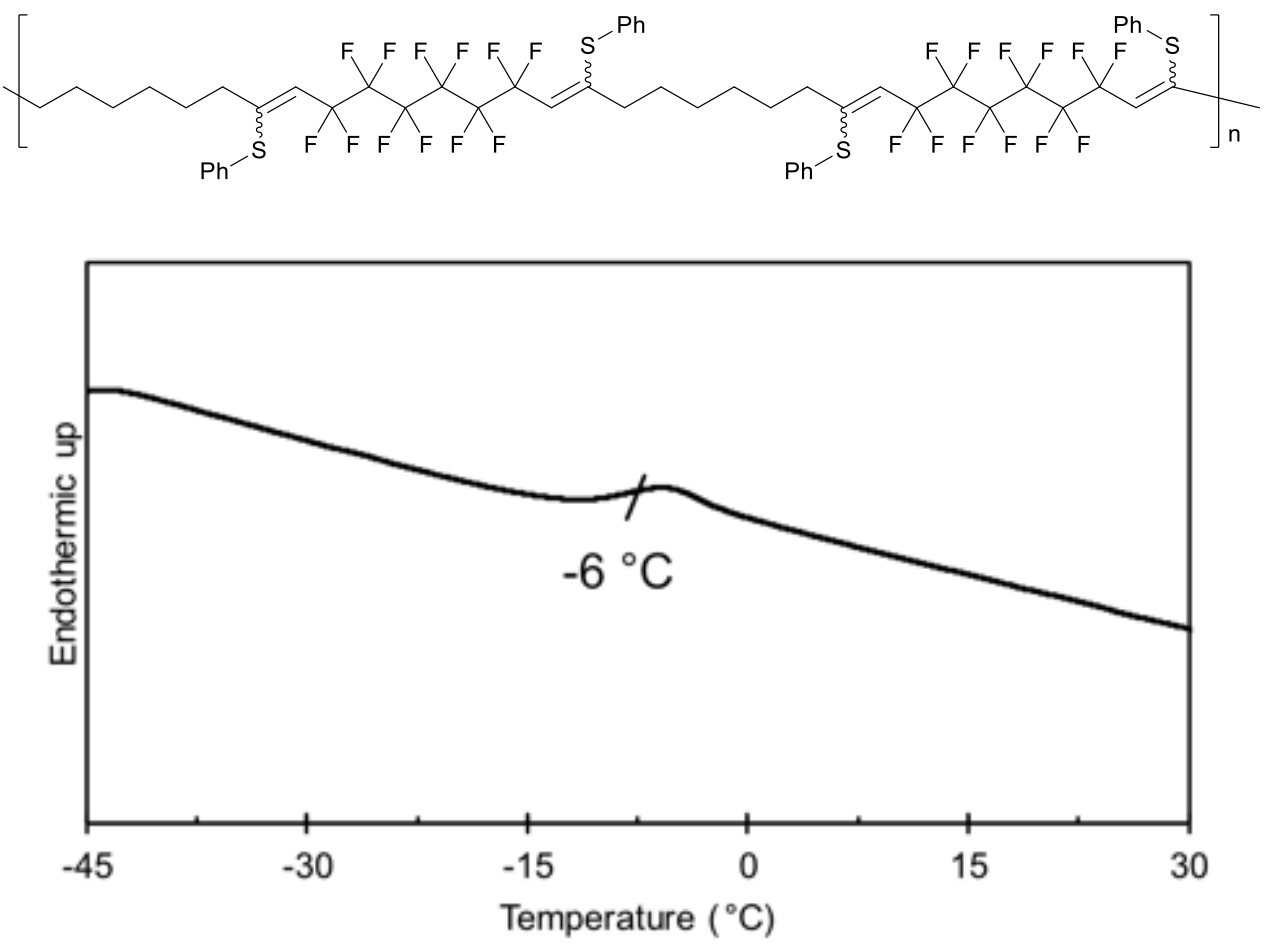
Kumada coupling of polymer 3,10 :
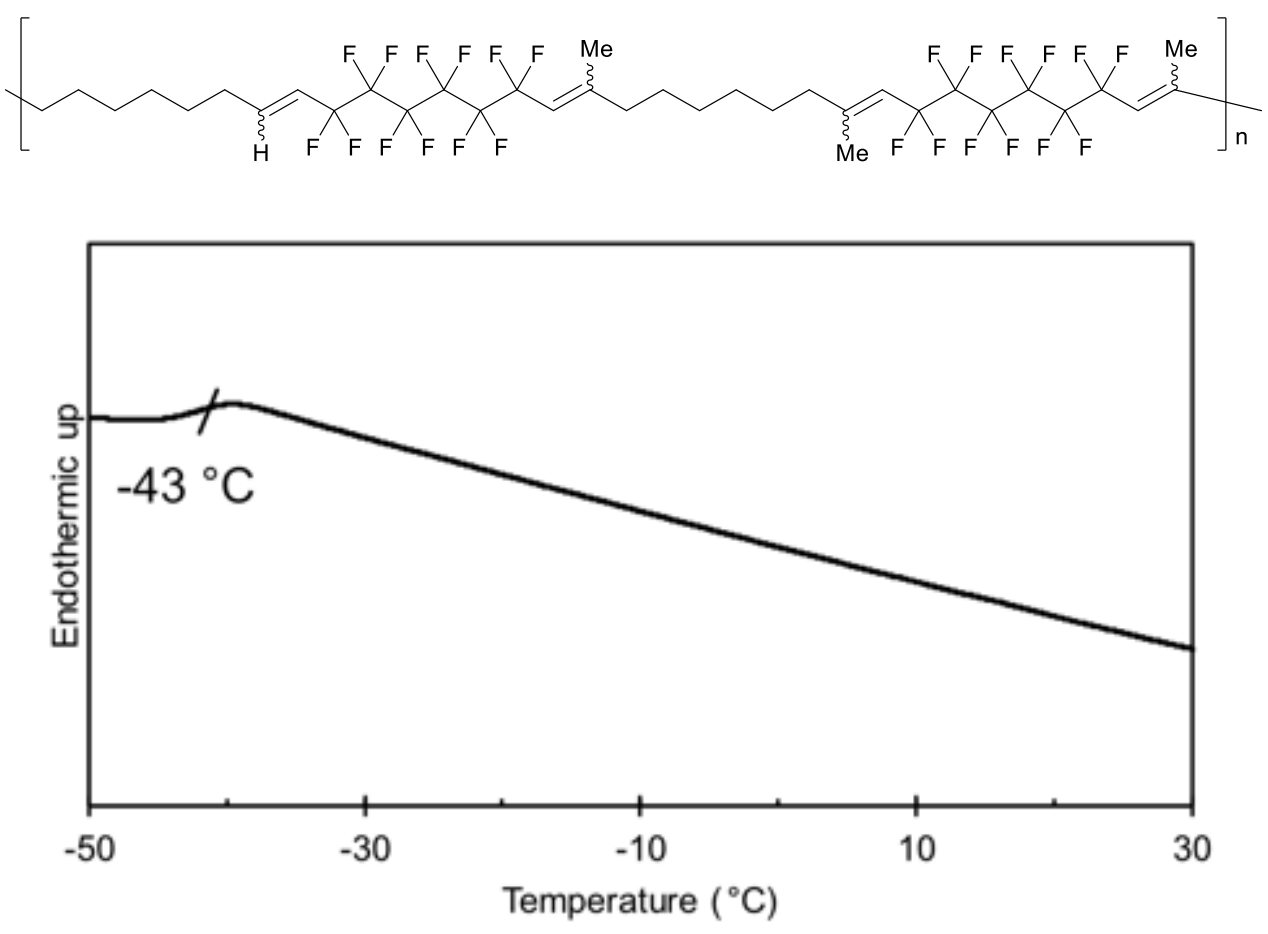


\section{Supporting References:}

(1) Pangborn, A. B.; Giardello, M. A.; Grubbs, R. H.; Rosen, R. K.; Timmers, F. J. Safe and Convenient Procedure for Solvent Purification. Organometallics 1996, 15 (5), 1518-1520.

(2) Xu, T.; Cheung, C. W.; Hu, X. Iron-Catalyzed 1,2-Addition of Perfluoroalkyl lodides to Alkynes and Alkenes. Angew. Chemie Int. Ed. 2014, 53 (19), 4910-4914. 